\title{
Stress reactivity, type $A$ behavior and vital exhaustion : searching for a psychobiological explanation of cardiovascular disease
}

Citation for published version (APA):

Lulofs, R. (1990). Stress reactivity, type A behavior and vital exhaustion : searching for a psychobiological explanation of cardiovascular disease. [Doctoral Thesis, Maastricht University]. Rijksuniversiteit Limburg. https://doi.org/10.26481/dis.19901005rl

Document status and date:

Published: 01/01/1990

DOI:

10.26481/dis.19901005rl

Document Version:

Publisher's PDF, also known as Version of record

Please check the document version of this publication:

- A submitted manuscript is the version of the article upon submission and before peer-review. There can be important differences between the submitted version and the official published version of record.

People interested in the research are advised to contact the author for the final version of the publication, or visit the DOI to the publisher's website.

- The final author version and the galley proof are versions of the publication after peer review.

- The final published version features the final layout of the paper including the volume, issue and page numbers.

Link to publication

\footnotetext{
General rights rights.

- You may freely distribute the URL identifying the publication in the public portal. please follow below link for the End User Agreement:

www.umlib.nl/taverne-license

Take down policy

If you believe that this document breaches copyright please contact us at:

repository@maastrichtuniversity.nl

providing details and we will investigate your claim.
}

Copyright and moral rights for the publications made accessible in the public portal are retained by the authors and/or other copyright owners and it is a condition of accessing publications that users recognise and abide by the legal requirements associated with these

- Users may download and print one copy of any publication from the public portal for the purpose of private study or research.

- You may not further distribute the material or use it for any profit-making activity or commercial gain

If the publication is distributed under the terms of Article $25 \mathrm{fa}$ of the Dutch Copyright Act, indicated by the "Taverne" license above, 
STRESS REACTIVITY, TYPE A BEHAVIOR AND VTTAL EXHAUSTION 


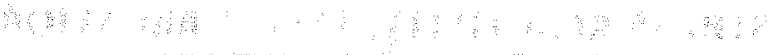

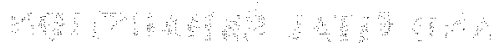




\title{
STRESS REACTIVITY, TYPE A BEHAVIOR AND VITAL EXHAUSTION:
}

\author{
searching for a psychobiological explanation \\ of cardiovascular disease
}

\author{
proefschrift \\ ter verkrijging van de graad van doctor \\ aan de Rijksuniversiteit Limburg te Maastricht, \\ op gezag van de Rector Magnificus, Prof. Dr. F.I.M. Bonke, \\ volgens het besluit van het College van Dekanen, \\ in het openbaar te verdedigen op \\ vrijdag, 5 oktober 1990 om 14,00 uur \\ door \\ Rutger Lulofs \\ geboren op 15 september 1945 te Vorden
}




\section{Promotores:}

Prof. dr. A.P.W.M. Appels

Prof. dr. G. Mulder (Rijksuniversiteit Groningen)

\section{Beoordelingscommissie:}

Prof. dr. M.A. Allessie (voorzitter)

Prof. dr. J.F. Orlebeke (Vrije Universiteit Amsterdam)

Dr. H. Schouten

Prof, dr. H.A.J. Struyker Boudier

Prof dr. M.W. de Vries

\section{CIP-DATA KONINKLIJKE BIBLIOTHEEK, DEN HAAG}

Lulofs, Rutger

Stress reactivity, type A behavior and vital exhaustion : searching for a psychobiological explanation of cardiovascular disease / Rutger Lulofs. - Maastricht :

Datawyse. - III.

Thesis Maastricht. - With ref. - With summary in Dutch.

IISBN 90-5291-031-6

SISO- 605.12 UDC [159.9:612]:616.1(043.3) NUGI 745

Subject headings: stress / psychological tisk factors / cardiovascular disorders. 


\section{TABLE OF CONTENTS}

Exaggerated stress respons as an explanation for increased risk Biological stress responses Activation of the PAC system

Conclusion

Emperical evidence for exaggerated stress reactions in type

\section{A individuals}

Subjects characteristics

\section{Experimental tasks}

Choice and measurement of dependent variables

Conclusion $A B$ typology

The experiments

Subjects

Experimental conditions

Dependent variables

\section{Procedure}

Statistical analysis 
Main effects of time pressure

Differences between type A and B subjects

Differences between exhausted and vital subjects

Conclusions

Results III. Effects of Critisism

Feedback on performance after a reaction task

Main effects of feedback

Differences between type A and $B$ subjects

Differences between exhausted and vital subjects

Feedback on performance during the quiz

Main effects of feedback

Differences between type A and B subjects

Differences between exhausted and vital subjects

Conclusions HIGH AND LOW RISK SUBJECTS

Subjects

Experimental conditions

Dependent variables

Procedure vital subjects 
3.5 Main conclusions and discussion

4.1. Review of the evidence found to support the main hypotheses and predictions

4.2 Our results compared to those of recent studies

4.2.1 Type A behavior

4.2.2 Vital exhaustion

4.3 Methodological critique

4.3.1 Critique on the narrative review method

4.3.2 Validity of the experiments on reactivity

4.3.2.1 The probability of type I and type II errors

4.3.2.2 A meta-analysis of experiments on reactivity

4.3.2.3 The power of the tests of a difference in reactivity between type A and B subjects

4.3.2.4 Number of F-tests employed

4.3.3 Consequences of methodological problems

$4.4 \quad$ Possible solutions

4.4.1 Increase in the power of the experiments

4.4.1.1 Increase in the expected difference in reactivity

4.4.1.2 Increase in the sensitivity of the experimental design

4.4.2 Reducing the variability in power

4.4.3 Reducing the number of statistical tests

4.5 Concluding remarks

REFERENCES

SUMMARY

SAMENVATTING 


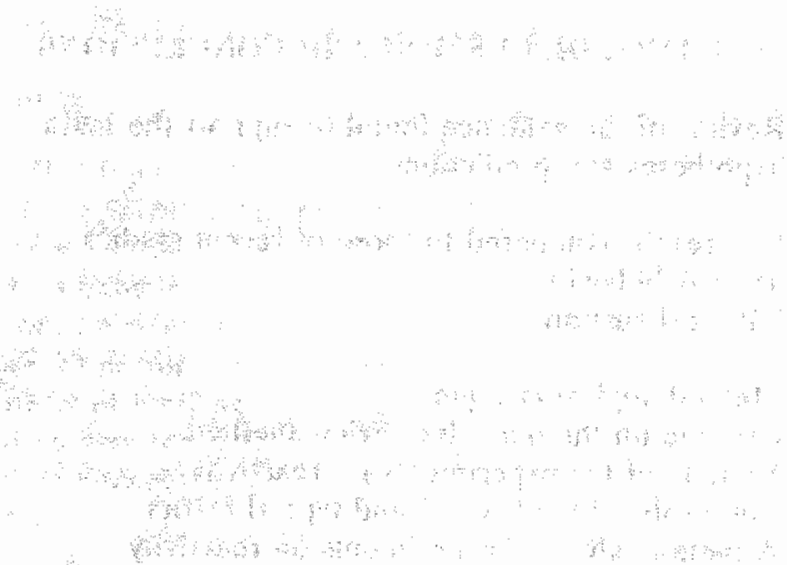


This thesis deals with the problem of how to explain, in biological terms, the association between cardiovascular disorders and two psychological constructs: type A behavior and vital exhaustion.

In the first chapter a description of the two psychological risk factors will be given, followed by a review of experiments in which an attempt was made to establish the biological link between these psychological constructs and the occurrence of cardiovascular disorders. The main research questions of this investigation are then presented. In the second and the third chapter two series of experiments are described.

In the last chapter the evidence found to support the tested hypotheses is summarized and compared to the findings of other investigations described in recent reviews. Furthermore, a methodological critique of both those investigations and the reviews is given. The chapter ends with some measures that can be taken to solve the methodological problems that were discussed.

\section{1}

\section{DESCRIPTION OF THE TWO PSYCHOLOGICAL RISK FACTORS}

Two psychological factors associated with increased risk of myocardial infarction have emerged from epidemiological studies: the type $A$ coronary prone behavior pattern and the syndrome of vital exhaustion.

Type A behavior. Type A behavior was originally described as "... an action-emotion complex that can be observed in any person who is aggressively involved in a chronic, incessant struggle to achieve more and more in less and less time and is required to do so against the opposing efforts of other things or other persons (...) stemming from a fundamental and irretrievable sense of insecurity about the intrinsic value of the personality involved." (Friedman and Rosenman, 1977). The major behavior elements are: competitiveness, a constant need to achieve, a sense of time urgency, impatience and easily aroused aggression or hostility. In 1978, during a conference, the status of type $\mathbf{A}$ behavior as an independent risk factor was established. The conclusion of a review panel that studied the strength of the association between type $\mathrm{A}$ behavior and coronary heart disease was that "... this risk is greater than that imposed by age, elevated values of systolic blood pressure and serum cholesterol, and smoking ...." (Cooper, 1981).

Vital exhaustion. More recently interest has developed in the syndrome of vital exhaustion as a possible risk factor. A number of investigations (Kuller et ail., 1972; Alonzo et al., 1975; Rissanen et al., 1978) showed that $30 \%-60 \%$ of the patients who suffered myocardial infarction or sudden death had complained of fatigue and weakness from several weeks to some months prior to their infarction or death. In two prospective studies (Shekelle and Ostfield, 1965; Brozek et al, 1966), high scores on 
the depression and hypochondria scales of the Minnesota Multiple Personality Inventory (MMPI) were associated with increased risk of cardiovascular disease. Intrigued by these findings, Appels (1980) developed a scale to assess these prodromal findings. A tentative dynamic model was used as a guideline for the formulation of the items for this scale. Feelings of tiredness, loss of energy, and demoralization were seen as the result of the following process. A basically insecure person strives very hard to be appreciated by those around him. In doing so, he uses up much energy, something which may owertax his regulatory systems, particularly when he is faced with a problem he cannot solve. This continuous self-imposed stress leads to a stage of exhaustion similar to that described in animals by Selye (1976). This stage also resembles the giving-up-given-up syndrome which has been described in humans by Engel (1968).

Sixty-three items based on these concepts were used as an initial pool that was tested psychometrically. Forty items were found to discriminate between a healthy control group and individuals who developed cardiac complaints or myocardial infarction within a ten-month follow-up period.

A cluster analysis showed that although some subclusters of items could be found, all items clustered together to such an extent that one scale score may be used. After inspection of the items and interviews with ten high-scoring, subjects the name "vital exhaustion and depression" seemed the most appropriate label for the item list. Besides being able to distinguish between healthy controls and future cases, the same items in another study also discriminated between controls and actual cases (Verhagen et al. 1980).

After these two studies the item pool was revised. The nondiscriminating items were dropped and others, mainly obtained from interviews with heart patients, were added. This new item pool was tested in a different prospective study. At the time our experiments were started, only the discriminating power of the new set of items was available. People scoring above the median had a fifteen times higher chance of being diagnosed as having imminent myocardial infarction than those scoring below the median (Appels, 1983). Although no evidence for the predictive validity of the revised item pool existed at that time, it seemed that, given the relative small change, it was justified to include the state of vital exhaustion and depression as a factor of interest in our experiments.

Possible interaction between the two risk factors. Beside the role vital exhaustion might play on its own, a second reason to include the construct in our studies was that, at lleast on theoretical grounds, type A behavior might promote the process that leads to a state of exhaustion. Glass (1977) has suggested that type A individuals have a higher need to control their environment than type B individuals. This higher need for control makes them more susceptible for an experience of loss of control and this will trigger the stage of exhaustion (Henry and Stephens, 1977). If this line of reasoning is correct the two concepts might not be independent and inclusion of both factors in an experiment is important in order to be able to assess the degree of this dependency and to be able to statistically control for it. 
After the establishment of the Type A behavior pattern as an independent risk factor in the development of cardiovascular disorders, an increasingly large number of studies appeared investigating the way in which biological mechanisms might be involved. The general idea behind these studies was that hyperreactivity defined as an exaggerated response to a stressor, might be the link between type $A$ behavior and coronary heart disease (CHD). Type A subjects were expected to overreact when confronted with a stressful situation.

Whether hyperreactivity should be expected in exhausted subjects is not clear. If we follow the model described in the previous section, in which the state of vital exhaustion is regarded as equivalent to Selye's stage of exhaustion (Selye, 1976), hyporeactivity rather than hyperreactivity might be expected. It is also possible that in exhausted subjects a different physiological system will be triggered than in type A subjects.

Hyperreactivity is potentially harmful since it may disturb the balance between the needs and the supply of the cardiovascular system. Several regulatory systems that are involved in guarding this balance - including the autonomic nervous system, neurohormonal circulation, and platelet activation - are affected by the stress response of an individual.

\subsubsection{Biological stress responses}

Evidence has been found, both from psychophysiological and from neuroendocrine studies, that different stress response patterns do occur. Mason (1971) was one of the first to draw attention to the fact that different patterns of hormonal release can be observed, depending on the kind of situation an individual is faced with. This idea has been taken up by others. Henry and Stephens (1977) make a distinction between the sympathetic-adreno-medullary (SAM) system and the pituary-adreno-cortical (PAC) system. In the next section we will discuss the conditions in which these two systems are triggered and the effects of the activation of the two systems on the cardiovascular system.

\subsubsection{Activation of the SAM system}

SAM activity is triggered when an individual strives to regain control in a situation in which this control is threatened Ursin and Knardahl (1985) found that individuals who were most successful in mastering difficult challenges had the highest levels of catecholamines. Also, in the work of Frankenhaeuser (Lundberg and Frankenhaeuser, 1980), a positive relationship was established between level of catecholamines and performance. They found that invested effort in an experimental task and levels of noradrenaline were positively related.

Thus, one may conclude that an association exists between striving for mastery and SAM activity. Since striving for mastery and control is a central issue for type A individuals an increase in SAM activity might be expected when they are confronted with a stressor. 
Effects of Increased SAM activity on myocardial demands. Due to the increased sympathetic drive and the increased levels of catecholamines heart rate increases and atrial and ventricular contractility increases, leading to an increased: workload on the myocardium. Particularly when increased SAM activity is not accompanied by increased physical activity, something which is usually the case when individuals are exposed to mental and emotional stressors, total peripheral resistance also increases (Williams et al, 1982). This leads to an increased afterload, which further increases the workload. The increase in total peripheral resistance is even more likely when, due to sustained increased SAM activity, structural changes in the vessel wall have taken place, leading to a reduced vessel wall-to-lumen ratio (Folkow and Rubinstein, 1966).

Effects of increased SAM activity on myocardial supply. Due to the reduced diastolic time interval that results from the increased heart rate, the blood supply to the myocardium is slightly impaired. This effect is counteracted by an active dilation of the coronary vessels. A more serious threat to the myocardial supply is the possible interference of SAM activity with the structure of the vessel wall and the possibility of SAM activity stimulating platelet aggregation. When platelets become activated, a number of substances are liberated. Among these are Thromboxane A2 and ADP, which, in turn, stimulate further activation of platelets and vasoconstriction (Buell and Eliot; 1980). Thus, when platelets become activated in coronary arteries, impairment of blood supply might occur, due to either obstruction by platelet aggregates or by constriction of the coronary vessels.

\subsubsection{Activation of the PAC system}

PAC activity is triggered when an individual has actually lost control over his situation and attempts to regain control are no longer made. The main biological changes that take place when the PAC system is activated are increased parasympathetic drive and increased liberation of ACTH, leading to the liberation of glucocorticoids from the adrenal cortex. Experimental evidence for the rellationship between loss of control and expectation of failure is given by Ursin and Knardahl (1985). They showed that parachute jumpers who were inexperienced and uncertain about their performance had higher levels of cortisol than those who were confident that they would land safely:

The description of the condition in which PAC activity is increased fits well with the dynamic model that describes exhausted subjects. Thus; it would seem reasonable to expect exhausted subjects to react with changes in the PAC system when confronted with a stressor.

Effects of increased PAC activity on myocardial demands. Little is known about the direct effents of increased levels of corticoids on the myocardium. Selye's observations (1971) that myocardial necrosis occurred in animals treated with glucocorticoids are difficult to interpret since SAM activity was increased at the same time. The increased vagal influence on the heart has little effect on contractility but leads to a slower heart rate and thus reduces myocardial demands.

Effects of increased PAC activity on myocardial supply. In contrast to the effect of increased sympathetic drive, increased vagal stimulation leads to a prolongation of the 
diastolic time interval, promoting myocardial supply. However, sustained high levels of glucocorticoids may indirectly promote the same changes in vessel wall structure as those promoted by catecholamines since glucocorticoids stimulate the production of catecholamines. Furthermore, there is a positive association between levels of ACTH and platelet activity (Warner et al., 1967). Thus, increased levels of ACTH might give rise to the same effect on platelet activity as increased activity of the SAM system.

\subsubsection{Conclusion}

Increased SAM activity, both sustained and temporarily, may lead to a negative balance between myocardial demands and myocardial supply and might, therefore, pose a potential risk. Temporarily increased PAC activity restores a more positive balance, but when PAC is chronically activated negative effects might be expected. Although the ultimate test of the hyperreactivity hypothesis requires a prospective study, a useful first step is to establish an association between hyperreactivity and type A behavior. In the next section we will examine the empirical evidence for this association.

\subsubsection{Empirical evidence for exaggerated stress reactions in type $A$ individuals}

The studies reviewed in this section will be restricted to those published up until 1980 since those were the studies that formed the basis of the experiments to be described in this thesis. The selected studies can be found in the literature list by looking at the references marked with [1]. In the last chapter the results of our experiments and those of the more recent studies will be discussed.

The results of a review of the earlier studies on reactivity indicated that signs of higher activation in type A subjects are an exception rather than a rule (Myrtek, 1983). The aim of this section is to see what distinguishes the studies that reported a larger increase in activation in type A subjects during exposure to a stressor from those that did not find a larger increase in activation in type A subjects.

The earliest studies (Friedman et al., 1960; Friedman et al, 1970) compared type A and $\mathrm{B}$ individuals, mostly middle-aged men that were classified using a structured interview. Measurements took place in a non-laboratory setting in which no explicit attention was given to the situation in which the measurements were taken. Nor were the biological variables that might be expected to differences between the two types specified before hand. When looking at the studies that have been published since, it becomes apparent that the way in which the subjects were selected, the conditions in which they were compared, and both the choice of the dependent variables and the way in which they were measured became increasingly important because of the influence they had on the resuits.

In the following sections the possible effects of these factors on the outcome of the tests of higher reactivity in type A subjects will be discussed in more detail. Test results were regarded as positive when type A subjects showed signs of a larger increase in at least one biological variable. Results were negative either when type B subjects showed signs of higher activation (something which seldom occurred) or when 
there was no difference between the two types on any variable. Often several tests, measuring differences in reactivity between the two types of subjects, were done within one experiment. When the statisticall analyses that were used allowed this, each comparison was counted as a separate test.

\subsection{2,1 Subjects characteristies}

Three main subject characteristics will be discussed: one is the selection instrument used to classify the subjects, another is the age and background of the subjects, and the third is whether males or females were compared.

Selection instrument. The majority of the studies used either the structured interview (SI), originally developed by Friedman and Rosenman (Rosenman, 1978) or the Jenkins Activity Schedule (JAS) a self-report measure developed to be used in prospective epidemiological studies (Jenkins, 1978). When using the interview, subjects are classified as $\mathrm{A} 1, \mathrm{~A} 2, \mathrm{X}, \mathrm{B} 3$, or $\mathrm{B} 4$, depending on the extent to which the type A characteristics are present. The JAS results in a score on a standardized scale with a mean of 0 and a standard deviation of 10 . The agreement between the two instruments has been found to be about $70 \%$ (Jenkins, 1978) with good agreement in the more extreme categories.

Age and sex. While the original description of the type A behavior pattern was based on the observations of predominantly middle-aged, middle class white males, quite a large number of studies have been undertaken using, primarily male, college students. Because the number of studies using females was small and because the use of male behavior as a reference for classification of females seems questionable, only studies on males will be discussed. In order to assess the influence of the other two aspects of selection, the studies were divided into four groups. In one group the SI was used to classify students, and in a second it was used to classify middle-aged men. In a third group the JAS was used on students, and in the last it was used on middle-aged men. Then the number of positive and negative results - the former being comparisons in which type $A$ showed a larger increase in activation and the latter being those in which either type B showed a larger increase or in which no significant differences were found - were counted for each of the four subgroups. The results are shown in table 1.2.1.

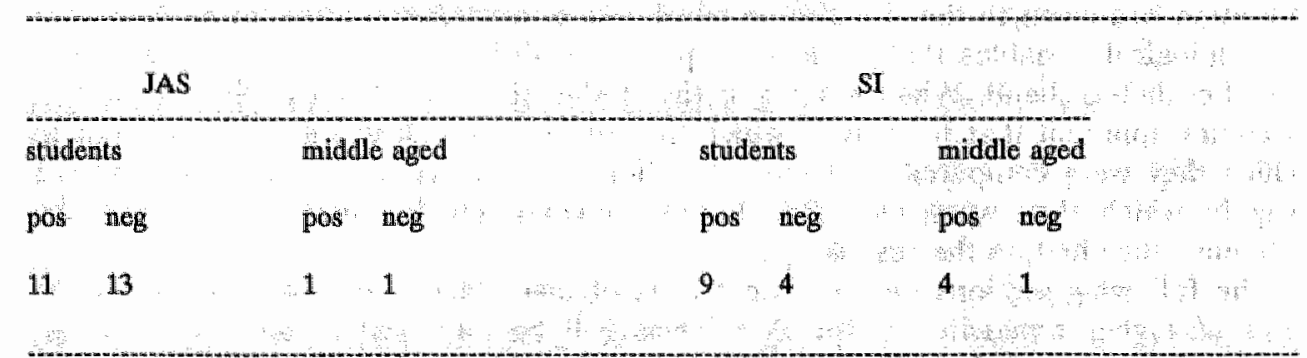

Table 1.2.1. The Influence of classification instrument and age on the number of posithe and negative tests of the difference in reactivity between type $A$ and $B$ subjects. 
Looking at the different methods of classification one can see that in the studies in which the SI was used, positive results were found in $72 \%$ of the cases; in those in which the JAS was used positive results were obtained in only $46 \%$ of the cases. This difference is, albeit marginally, significant $\left(\mathrm{Chi}^{2}=2.94 \mathrm{df} 1, .05<\mathrm{p}<.10\right)$. The proportion of positive results was also somewhat higher in middle-aged men than in students: $71 \%$ vs $54 \%$. Since the number of studies using middle-aged men was fairly small, the effects of the other factors influencing outcome might not be evenly distributed over this factor. Therefore, this difference is probably not very reliable. Statistically is was not significant either $\left(\mathrm{Ch}^{2}=.71\right.$ df $\left.1, \mathrm{p}<.40\right)$.

\subsubsection{Experimental tasks}

A wide variety of tasks have been used in which type A and type B subjects were compared with each other. While in all studies the tasks were chosen in order to elicit some of the core aspects of the type A behavior pattern a distinction can be made between those studies in which it was assumed that the subjects would show these behaviors spontaneously and those in which it was thought necessary that the typical type A behavior had to be provoked. In the first type of studies, instructions about the need for excellent performance were not given; nor were extra incentives used. In the second type of studies, tasks were either made very difficult, resulting in failure to obtain good results, or subjects were told they did not perform as well as could be expected. Another way of inducing failure was to make sure subjects would lose when competing with another person by using a well trained opponent. In addition, hostillity was sometimes evoked by making harassing remarks to the subject. When comparing the effects of explicit vs non-explicit elicitation, as shown in table 1.2.2 one can see that in the latter studies fewer positive results were obtained than in the former: $27 \%$ vs $60 \%\left(\mathrm{Chi}^{2}=3.37\right.$ df $\left.1, .05<\mathrm{p}<.10\right)$.

\begin{tabular}{c|cccc} 
& explicit & & non-explicit \\
\hline pos & neg & pos & neg \\
& 15 & 10 & 3 &
\end{tabular}

Table 1.2.2. The influence of explicit vs nonexplicit llicitation of type $A$ behavior on the number of positive and negative tests of the difference in reactlily between type $A$ and $B$ subjocts.

It should be noted that the total number of comparisons presented in table 1.2 .2 is not necessarily the same as that in table 1.2.1 since not all tasks could be classified unequivocally. For instance the finding that type A subjects had a higher adrenaline excretion during forced inactivity (Frankenhaeuser et al., 1980b) was not included in this comparison because no actual task was performed. However, this finding lends support to the assumption that when core aspects of type A behavior are explicitly 
elicited, in this case impatience by providing a very boring situation, differences in activation can be seen more clearly.

\subsubsection{Choice and measurement of the dependent variables}

As mentioned above, only a globally defined larger increase in activation was expected in type A subjects in the earlier experiments. The increased activation was thought to result in an increase in heart rate and blood pressure and in the release of stress related hormones like catecholamines and cortisol, reactions belonging to the alarm stage of the stress response according to Selye (Selye, 1976). However, as already discussed in the previous section, depending on the way in which an experimental stressor is evaluated, a different reaction pattern may be elicited and thus different variables may be involved. This was sometimes underestimated in the studies reviewed leading to the wrong expectation of the kind of activation pattern. Although often not enough variables were measured to fully assess the possible differences in activation patterns, in most cases measurements of heart rate and of systolic and diastolic blood pressure were made. This made it possible to determine which of these variables most consistently showed differences between type A and B subjects. It also shed some light on the activation pattern that might prevail in type $A$ individuals.

A second point which has not always been dealt with systematically, is the expected duration of the increased activation. In some studies a sustained high level of activation was apparently expected since values of the biological variables were averaged over long periods or were taken at the end of stressful tasks. In other studies responses of short duration were investigated. The duration of high levels of activation is an important factor for the following reason. A sustained high level of activation. will trigger different compensatory mechanisms than temporary high levels of activation. These different compensatory mechanisms will, in turn, lead to differences in the activation pattern itself. Therefore, different pathological mechanisms must be considered when sustained differences in activation between type $A$ and $B$ subjects are found rather than temporary one's.

In table 1.2.3 the effect of the choice of the dependent variables and that of the measurement of sustained versus temporary high levels of activation on the outcome of the studies is shown. Studies in which tasks lasting more than two minutes were used and in which activation scores were either based on mean values during the task or measured immediately after the task was completed were classified as investigating differences in sustained activation. Studies in which tasks lasting two minutes or less were used or in which longer tasks were used that allowed for the assessment of initial changes were classiffied as investigating differences in temporary activation.

The first thing one notices when looking at table 1.2 .3 is that type $A$ and $B$ subjects differed more often during temporary higher levels of activation than during sustained higher levels of activation in heart rate $\left(\mathrm{Chi}^{2}=16.56 \mathrm{df} 1, \mathrm{p}<.001\right.$ ) and, marginally, in systolic blood pressure ( $\mathrm{Chi}^{2}=2.70$ df $1, \mathrm{p}<.11$ ). Secondly, systolic blood pressure seems to be the most sensitive variable although during temporary activation heart rate is equally sensitive. Finally diastolic pressure is seldom higher in type A subjects both during sustained and during temporary activation.

One is tempted to conclude that the observed higher activation pattern in type A 
Sustained activation

Temporary activation

\begin{tabular}{|c|c|c|c|c|c|c|c|c|c|c|c|}
\hline \multicolumn{2}{|l|}{ SYS } & \multicolumn{2}{|l|}{ DLAS } & \multicolumn{2}{|c|}{ HRATE } & \multicolumn{2}{|l|}{$\mathrm{SYs}$} & \multicolumn{2}{|l|}{ DIAS } & \multicolumn{2}{|c|}{ HRATE } \\
\hline pos & neg & pos: & neg & pos: & neg & pos & neg & pos & neg & pos & neg: \\
\hline $17 \%$ & $\begin{array}{l}8 \\
53 \%\end{array}$ & $\frac{2}{14 \%}$ & $\begin{array}{l}12 \\
86 \%\end{array}$ & $\begin{array}{l}3 \\
13 \%\end{array}$ & $\begin{array}{l}21 \\
87 \%\end{array}$ & $\begin{array}{l}10 \\
77 \%\end{array}$ & $\begin{array}{l}3 \\
33 \%\end{array}$ & $\frac{2}{15 \%}$ & $85 \%$ & $\begin{array}{l}11 \\
79 \%\end{array}$ & $\begin{array}{l}3 \\
21 \%\end{array}$ \\
\hline
\end{tabular}

Table 1.2.3: The influence of sustained us temporany activation on the number of pasitive and negative tests of the difference in reactivity between type $A$ and $B$ subjects.

subjects is caused by a larger inerease in beta-adrenergic receptor stimulation, leading to an increase in systolic blood pressure and heart rate. In most cases the effects disappear within a few minutes, either because of an increased vagal activity, causing heart rate to slow down and systolic pressure to remain fairly high, or by straightforward habituation to the task, resulting in reduced beta-adrenergic stimulation. In the latter case, both heart rate and systolic pressure subside to levels comparable to those of type B subjects. The evidence for increased alpha-adrenergic receptor stimulation in A type subjects, which one would expect when they excrete a larger amount of noradrenaline, is not very strong. The combination of higher levels of both systolic and diastolic pressure which would result from this kind of stimulation is found less often than higher levels in systolic pressure alone. Higher levels of diastolic pressure, in combination with normal levels of systolic pressure and heart rate resulting from compensatory mechanisms, were never found. However, on the basis of this meta analysis one can only speculate on changes in regulating mechanisms. Without the results of experimental studies designed to test the question of changes in regulation, it is not really possible to decide which regulatory mechanisms are involved.

\subsubsection{Conclusion}

That signs of larger activation in type A subjects are the exception rather than the rule seems too strong an assertion.

The likelihood of larger activation in type A subjects seems to depend on the way in which the subjects are selected, the type of stressor used and the choice of the variables used to assess the changes in activation. The number of studies thus far referred to is too small to be able to assess the effects of these three factors indepen dently, so any conclusion about the effects of these factors should be regarded as preliminary.

Still a number of recommendations may be given and some interesting research areas can be listed.

The classification instrument for type A behavior that seems to result in the clearest difference in reactivity is the SI although, for practical reasons, selection of subjects on 
the basis of extreme JAS scores is a reasonable alternative.

The fact that situations that explicitly elicit the core behaviors in type A subjects were more successful in showing a difference in reactivity than more neutral situations would suggest that it is a specific way of dealing with certain events or, in other words, a specific coping strategy that distinguishes type A individuals from their type B counterparts. This opens the field for more specific research into the kinds of situations that trigger the core elements of type $\mathrm{A}$ behavior.

There are indications that the way in which type A subjects deal with stressors is usually accompanied by an exaggerated increase in SAM activity. Whether this is invariably the case or whether situations can be found in which type A subjects react with a larger increase in PAC activity is another interesting area of research.

Finally it would seem that differences in activation are not very large nor do they last very long. The first point is illustrated by the fact that significant differences were mainly found when using a very sensitive statistical analysis. Just comparing activation scores during task performance seldom resulted in differences between the two types. The fact that when higher activation was found it was usually of short duration illustrates the second point. It is possible, however, that the differences in reactivity disappeared during long-lasting tasks once reflexive counter-regulation brought the initial higher level of activation in type $A$ subjects back to a level comparable to that of type B subjects. For instance, after several minutes heart rate in type A subjects might have dropped to a frequency similar to that in type B subjects not because of the disappearance of a more pronounced activation of their SAM system but because of increased vagal stimulation brought on by the baro-reflex.

Some of the questions raised above were studied in the experiments that were performed for this thesis.

In this section the main research topics are described. The more specific hypotheses are presented at the beginning of the second and the third chapter in which the experiments are described in detail.

\subsubsection{Stress reactions in type $A$ and type $B$ individuals}

The first topic to be studied is the type of biological response pattern that might be involved in the larger reactivity in type A subjects.

The general hypothesis is that type $A$ subjects show a langer increase in sympathetic adrenal medullary (SAM) activity than type $B$ subjects when exposed to a stressor. As discussed in the previous section not only the SAM system but also the PAC system may be involved in stress reactions. Given the paucity of data on differences in reactivity of the PAC system between type A and type B subjects, no specific prediction is made. However, an attempt is made to answer the following question: Are there conditions in which type $A$ subjects show a langer increase in pituitary adrenal cortical $(P A C)$ activity than type B subjects?

The second topic investigated in the study concerns the possible link between 
hyperreactivity in type A subjects and the saliency of a task i.e. the degree which a task has certain features that trigger the type $A$ behavior pattern. The idea that saliency might play a role is based on the observation that the explicit elicitation of core characteristics most often leads to differences in reactivity.

Two questions are of interest. The first is whether experimental manipulation of the degree of saliency will affect hyperreactivity in type A subjects. In trying to esthablish a dose-response relationship between task saliency and hyperreactivity in type A subjects a more refined test of the role of task saliency can be made. The second question is which particular experimental situation will trigger the core characteristics most effectively? Quite a number of different experimental tasks were used in the experiments reviewed. It is not always clear which of the core elements were supposed to be triggered and to which extent this happened.

\section{3 .2}

\section{Stress reactions in exhausted and vital individuals}

As explained in section 1.1, the concept of vital exhaustion resembles the third stage of Selye's stress model (Selye, 1976) and also that of Henry's conservation-withdrawal reaction (Henry and Stephens, 1977). Thus, the same biological reactions that accompany these two conditions might be expected: This leads to the general hypothesis that exhausted subjects show a larger increase in activation of the PAC system when exposed to a stressor.

Given the paucity of data regarding differences in SAM activity between vital and exhausted individuals, no specific hypotheses are formulated with respect to this response system. Instead, a more descriptive approach is taken. Our main interest is to see whether vital and exhausted subjects react differently in SAM activation to the various experimental conditions.

\subsubsection{Possible interaction between level of exhaustion and AB typology}

Since loss of vitality is expected to lead to a different stress reaction than being classified as a type A subject and since the two concepts might not be independent we want to see in which way the two risk factors might interact. No specific predictions are made but the way the data are analyzed allow exploration of this point.

\section{4}

\section{THE EXPERIMENTS}

Two series of experiments were performed. These experiments were partly exploratory and partly designed to test more specific hypotheses. They have the same basic design. Subjects are divided into high and low risk groups on the basis of their scores on the two psychological risk factors. These subgroups are exposed to several stress evoking situations and a number of physiological, hormonal and psychological variables are measured during these exposures. The values of these variables are compared with those obtained during a neutral condition.

In the next two chapters, the two series of experiments will be described in more detail. 


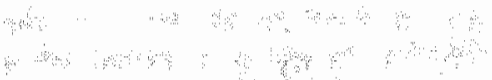

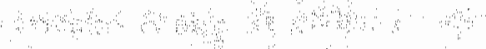
A

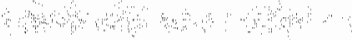

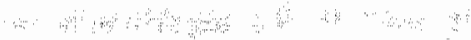

म क कि क्ष
अ

$\sin$

$\therefore$

क्ष.

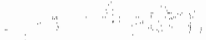

a

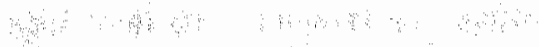

Q4 :

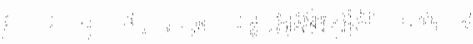

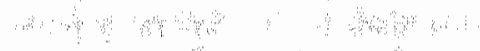

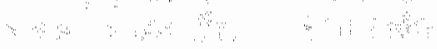

B.

$\because$

त

\&.

$\cdots$

a $\quad 2 \quad 3$

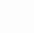

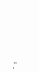


The experiment that will be described in this chapter was designed to test a number of hypotheses. These are listed in section 2:1. A detailed description of the way in which the experiment was carried out is given in section 2.2 and the results are presented in section 2.3 to 2.6 . Finally the way in which the results may be linked to the hypotheses is discussed in the concluding section 2.7.

\section{1}

\section{HYPOTHESES}

One hypothesis was derived from the first main question stated in section 1.3.1. Furthermore, three hypotheses were formulated that addressed the issue of task saliency.

The first hypothesis, derived from the first main question is that type $A$ subjects show a larger increase in subjective and in the physiological activation scores that reflect $S A M$ activity than type B subjects when exposed to a stressor.

The second hypothesis is that the difference in physiological activation between type $A$ and $B$ subjects can be seen more clearly at a medium level of time pressure than at high or low levels of time pressure. This hypothesis stems from the observation made by Matthews (Matthews, 1982) that situations that are moderately difficult and/or challenging provide the best opportunity to trigger type A behavior.

The third hypothesis is that the difference in subjective and physiological activation between type $A$ and $B$ subjects is seen more clearly after criticism than before feedback is given. This hypothesis is based on the fact that criticizing the performance of leads to increased effort in type A subjects to perform better (Glass, 1977, Brunson and Matthews, 1981) and that higher physiological activation has been found in type A subjects during a tasks in which they were continuously urged to perform faster and more accurate (Dembroski et al., 1977).

The fourth hypothesis is that type A subjects show higher levels of physiological activation than type $B$ subjects during situations in which active coping is inappropriate. It is expected that this will be particularly unpleasant for type A subjects since it deprives them from their normal way of coping: to be actively engaged in changing an unwanted situation. Some experimental evidence for this idea does exist. Frankenhaeuser et al. (1980a), and Lundberg and Forsman (1979) found that type A subjects are more aroused and feel more distressed than type $B$ individuals when they are deprived of work.

Regarding the effect of exposure to a stressor on exhausted subjects, no specific hypotheses are formulated with respect to task saliency. Only a more general hypothesis is tested: the exhausted subjects react more strongly on the variables that reflected 
PAC activity than vital subjects when exposed to a stressor.

\subsubsection{Subjects}

A general invitation to participate in research on behavioral aspects of cardiovascular disorders was sent to all male inhabitants of the city of Maastricht born in 1940 and 1941. This means that they were between the ages of 39 and 41 years at the time of the experiment. Of the approximately 2000 males about two thirds expressed their willingness to participate. Both the Dutch version of the Jenkins Activity Scale (JAS), which measures type $A$ behavior, and the Maastricht Questionnaire (MQ), which measures the degree of vital exhaustion, were sent to a randomly chosen sub-sample of 400 men. 238 of these men returned the questionnaires.

The Dutch JAS consisted of 36 items that were validated against the standardized interview in the Kaunas-Rotterdam study by Appels (Appels et al., 1982). The MQ was used in its provisional form and consisted of the 40 items that had been discriminating between future cases of coronary heart disease and a healthy control group (Appels, 1980), and 10 additional items that were constructed on the basis of interviews with coronary patients.

Subjects for the experiment were selected on the basis of their JAS scores. Scores of the 238 men that returned the questionnaire ranged from -25 to 45 with a mean score of 9 and a standard deviation of 15 . Those individuals with scores above the mean plus one standard deviation were designated as type A subjects, while those having scores lower than the mean minus one standard deviation were designated type $B$ subjects. Complete data were available for fourteen type A and fourteen type B subjects. On the basis of their scores on the Maastricht Questionnaire, these twentyeight subjects could also be divided into fourteen vital and fourteen exhausted subjects, using the median score of the total group of 238 subjects as a cutoff point. Table 2.2.1 shows the resulting four subgroups of subjects.

\begin{tabular}{lccc} 
& JAS score & MQ score & Nr \\
\hline Exhausted type A & $29.6(4.8)$ & $4103.9(23.7)$ & 10 \\
Vital type A & $32.0(7.5)$ & $70.5(1.9)$ & 4 \\
Exhausted type B & $-14.5(4.6)$ & $97.8(27.4)$ & 4 \\
Vital type B & $-10.2(3.4)$ & $64.5(5.4)$ & 10
\end{tabular}

Table 2.2.1: Mean scores and standard deviation on the JAS and the MQ of the four subgroups of subjects in the first experiment. 
One can see that the number of subjects was not equally distributed across the four subgroups. The type A subjects were more often exhausted and the type B subjects more often vital. To test whether the two risk factors were independent the Spearman rho correlation coefficient between MQ scores and JAS scores was calculated because the MQ scores were not normally distributed. This correlation was 0.40 which is significant at $\mathrm{p}<.05$ and indicates that the two risk factors are not independent. This finding has consequences for the statistical analysis which will be discussed in section 2.2.5.

None of the selected subjects had current or prior cardiovascular complaints or used any form of medication, according to their own account.

\subsubsection{Experimental conditions}

The selection of stress evoking situations was mainly based on the characteristics of the type A behavior pattern. Those situations that might trigger the core elements time pressure, competition and impatience were chosen and, where possible, different levels of saliency were used. Time pressure was manipulated in a reaction task in which different time limits were used within which the subjects had to react. Competstion was manipulated by providing either positive or negative feedback about the performance of the subjects. Impatience was induced by deliberately letting the subjects wait. To create a neutral condition that could be used as a baseline condition, a separate session was scheduled in which video documentaries on various non arousing themes were shown. The experimental conditions are now described in more detail.

Reaction task. A dual choice reaction task was presented in which different time periods were used within which a reaction had to be made. This task was expected to induce time pressure and to be challenging since continuously feedback was provided. High and low tones $(1600 / 500 \mathrm{~Hz})$ were presented randomly with a mean interval of 2.5 seconds. The subject had to react as quickly as possible by lifting his finger from a resting button and pushing one of two buttons that corresponded with the high or low tone after which he had to return his finger to the resting button. The different time limits were $600,520,460,420$ and 400 milliseconds. When the subject reacted by pressing the appropriate button within the allowed time, a green light would flash briefly. A red light would come on when he either reacted too late or pressed the wrong button. Five one minute series of tones were each followed by a resting period, also lasting one minute. Each series had a different time limit; the order in which the series were presented was randomly chosen for each subject. This task was presented twice, the first run without an external frame of reference regarding the level of performance and a second run after the subject had been told how his performance was related to that of other people.

Quiz. To increase the competitive drive of type A subjects by the presence of another person, a general knowledge test, consisting of questions covering topics from history, politics and regional issues, was administered by an associate experimenter seated in front of the subject. The subject was told that he had to correctly answer as many 
questions as possible. The associate read the questions from cards during a period of five minutes and made either positive or negative remarks about the answers given by the subject dependent on the feedback condition the subject was in. See Feedback on performance for further details.

Feedback on performance. To manipulate the expected level of performance, two different kinds of feedback were given after the first reaction task and during the quiz. After the first run of the reaction task half of the subjects were told that they had performed well above average and were encouraged to keep up their performance: The other half were told that they had performed well below average and were urged to improve their performance. During the quiz the subjects who had been praised on their performance on the reaction task were now also praised for every correct answer and told not to worry if they could not come up with the right answer. The other subjects were led to believe that all questions could be answered easily and they were criticized whenever they were unable to answer a question; no remarks were made when they answered correctly.

Waiting for a venous puncture. In order to assess blood platelet activity, a venous puncture had to be performed at the end of each session. To induce feelings of impatience during the experimental session, the first experimenter announced that she was going to get the person who was qualified to do the venous puncture. She told the subject that this person was waiting outside but, in fact, five mimutes elapsed before she retumed and the puncture was performed.

Video documentaries. Partly in order to establish a baseline period for the hormone measures and the blood platelet activity and also to circumvent the difficulties inherent in using short pre- or post-experimental resting values as an estimate for the basal level of physiological activation, a control session was included in which the subject was presented with two T.V. documentaries, each lasting approximately twenty-five minutes.

\subsubsection{Dependent variables}

Two groups of dependent variables were measured: behavioral and biological.

The behavioral variables were subdivided into self reported state and performance variables. The state variables were measured in order to assess the way in which the stress evoking situations were experienced. The performance variables were measured to investigate whether high and low risk subjects would perform differently under stress. Performance was expressed in speed and accuracy of reaction.

The biological variables were chosen with respect to a number of mechanisms that might be involved in the etiology of cardiovascular disorders. These mechanisms include increased activation of the autonomic nervous system, increased levels of circulating stress hormones and changes in blood platelet function.

Subjective state variables. Subjective state was assessed with visual analog scales. Visual analogue scales were chosen because they are more sensitive to small changes in a repeated measurement design. The subject had to indicate to what extent he felt tense, irritated, insecure, inpatient, tired, interested in the task, how well he had been 
able to concentrate and how much effort he had spent. For each item an "X" was to be placed along a $100 \mathrm{~mm}$ long line. The end points were defined as the maximum and minimum levels ever experienced by the subject. The indicated position on the scales was measured in millimeters. $A$ factor analysis was performed both on these raw data and on the data from the other experiment in which the same scales were used (Riedel, 1984). A two-factor structure emerged that was stable over the different situations and similar for type A and B subjects. The items irritation; tension, impatience and insecurity contributed to the first factor which was labeled "distress". The remaining items, ie effort, concentration and interest, contributed to the second factor, which was labeled "effort". A confirming factor analysis furthermore showed a good correspondence with the Effort and Distress factors used by Lundberg and Frankenhaeuser (1980). The mean of those items belonging to each factor was used as a distress score and an effort score.

Performance variables. Performance during the reaction task (i.e. reaction time and type of response) were measured with a tailor made apparatus and stored on a PDP Mine-11 computer. Reaction time was measured in milliseconds. Three types of responses were differentiated: a reaction within the time limit on the correct button was labeled "correct", a reaction within the time limit on the wrong button was labeled "incorrect" and a reaction that took longer than the time limit was labeled "missed". Mean reaction time and mean percentage correct responses were calculated for each task minute of the reaction tasks.

The performance measures during the quiz consisted of the total number of questions asked and the percentage of correct answers.

Physiological variables. Inter beat interval (IBI), influenced by both the sympathetic and parasympathetic nervous system, pulse arrival time (PAT), influenced predominantly by the sympathetic branch of the autonomous nervous system (Weiss et el, 1980 ) and, in a post hoc analysis, heart rate variability (HRV) being mainly influenced by the parasympathetic branch of the autonomous nervous system (Mulder, 1980) were selected as the physiological indices of SAM and PAC activity. Respiration rate was also measured, mainly as a control variable in order to detect gross disturbances during the registration. The physiological recordings were made on a Beckman R-611 polygraph and were stored on magnetic tape for further analysis. A bipolar ECG was recorded with $\mathrm{Ag} / \mathrm{AgCl}$ electrodes, using a modified $\mathrm{V}$ II lead in order to obtain a well-defined R-wave. An ear densitogram was recorded with a Hewlett Packard no 780-16 ear plethysmograph, Respiration was recorded using a strain gauge placed around the abdomen.

From the recordings of the ECG and the ear densitogram, inter beat intervals (IBI) and pulse arrival times (PAT) were determined on a beat-to-beat basis with an accuracy of one millisecond. IBI was defined as the time between two successive $\mathrm{R}$-waves and PAT as he time between an R-wave and the occurrence of the maximum value in the ear densitogram in the same beat. Respiration rate (RR) was determined by counting the number of respirations in one-minute periods. Then one-minute mean values were obtained for IBI, PAT and RR. Mean values of IBI, PAT and RR during venous puncture were obtained by averaging over the total duration of this procedure: After the experiment had been executed the possibility arose to analyze the data in 
Groningen, using the INSPAN program (Mulder, 1979), in order to obtain a spectral measure of heart rate variability. A spectral analysis was done to obtain a measure of heart rate variability with a frequency between 0.07 and $0.12 \mathrm{~Hz}$ using the beat-to-beat IBI data. These analyses were done on one minute periods during the reaction task, which is rather short to obtain a reliable power assessment for the lower frequencies but just adequate for the medium band, and on blocks of five minutes during the other tasks and during the T.V. documentaries.

Platelet activity. The blood samples, obtained through venous puncture, were immediately processed to measure aggregation velocity according to Born (1962). Platelet-rich plasma was prepared by spinning the blood for 10 minutes at $180 \mathrm{~g}$. Aggregation was measured with a Payton aggregometer, using collagen as an activating agent. Several tests were performed on each sample. In the first test, a collagen concentration of 10 $\mathrm{mg} / \mathrm{ml}$ was used and, in each subsequent test, the concentration was haived until aggregation could no longer be observed. The collagen concentrations that were used in the aggregation tests ranged from undiluted to eight times diluted. Aggregation velocity was expressed as the tangent of the angle of the line drawn through the steepest part of the aggregation curve. Aggregation velocity was weighted for the amount of dilution by multiplying the tangent by the degree of dilution and the maximal value thus obtained was used as an index of platelet activation. The larger this index was the more sensitive the platelets were to stimulation with collagen.

Hormonal data. After both sessions urine samples were taken covering the time the subjects had spent in the laboratory by asking them, upon arrival at the laboratory, to empty their bladders and, after the lab session was over, having them void again. The samples obtained at the end of the sessions were acidified $(\mathrm{pH}<4)$ and stored at -18 $\mathrm{C}$ until biochemical analyses was performed. Urine concentrations of metabolites of catecholamines and of cortisol, respectively Vanilla Mandelic Acid (VMA) and 17hydroxycorticosterone (17-OHCS), were determined. Creatinine concentration was also measured to correct for possible differences in kidney filtration function. From the urine samples, processed as described above, the ratios V.M.A./Creatinine and 17-OHCS/Creatinine were calculated, resulting in an index for sympathetic-adrenal-medullary and pituary-adrenal-cortical activity for each session.

\subsubsection{Procedure}

The experiment was carried out in a brightly illuminated soundproof room. The subject was seated in a straight-backed chair and separated from the recording equipment and the experimenter by a sound-absorbing screen.

The tasks were presented during the first experimental session. One week later; a separate base line session was held during which two video films on non arousing themes were presented. The tasks were presented in a fixed order.

A schematic representation of the procedure followed during the task session is given in figure 2.2.1.

After the subjects had arrived and emptied their bladder, they were led to the laboratory where preparations for the physiological recordings were made (1). When all recordings were satisfactory, the subjects filled in the visual analog scales for the 
first time. They were asked to indicate how they felt at that moment. Then, the reaction task was explained and a trial run of approximately five minutes was made during which all the different time limits were used successively (2). Following this trial run was a one-minute resting period. This was then followed by the first reaction task, which lasted ten minutes (3). When they had finished this task, half of the type A and half of the type B subjects were given positive feedback (4a), while the other subjects were given negative feedback (4b). Then, the reaction task was repeated, using the same order of time limits as in the first presentation ( $5 \mathrm{a}$ and $5 \mathrm{~b}$ ).

(1) Preparation

+ baseline

(2) Training

reaction task

(3) First run

reaction task

(4a) Positive feedback

(5a) Second run reaction task

(6a) Quiz pos, fdbk. (4b) Negative

feedback

(5b) Second run reaction task

(6b) Quiz neg. fdbk.

(7) Waiting period

(8) Venous puncture

(9) Resting period

Figure 2.2. I: Procedure of the task session in experiment I.

After completion of the second reaction task, the quiz was introduced. The subjects who had received positive feedback were told that the next test consisted of fairly difficult questions and that they were not expected to answer all of them correctly. The subjects were told not to worry if they did not know the right answer and were told just to ask for the next question. During the quiz, they were encouraged and praised (6a). The subjects who had received negative feedback were told that the next test consisted of fairly easy questions that could be answered correctly by almost anybody. During the quiz, these subjects were criticized for every wrong answer they 
gave (6b). After the quiz, all subjects had to wait five minutes for the venous puncture (7). The duration of the yenous puncture (8) varied between two and four minutes. A one-minute resting period (9) concluded the stress session. The total duration of the stress session was about ninety minutes.

The control session consisted of seven steps. In figure 2.2 .2 the schematic representation of the procedure is shown.

The first part of the session was similar to that of the stress session. Upon arrival, the subject were asked to empty their bladder and then preparations were made for the physiological recordings (1).

(1) Preparation

for recording

(2) Pre film resting period

(3) First

film

(4) Between film

resting period

(5) Second

film

(6) Venous

puncture

(7) Post film

resting period

Figure 2.2.2: Procedure of the film session in experiment I.

When all recordings were satisfactory, the subjects filled in the psychological state scales and were given a one-minute resting period (2), after which the first documentary was shown (3). When this film was finished the subjects filled in the scales again and a second one-minute resting period followed (4). Then, the second documentary was shown (5), again followed by the filling in of the scales. This time the venous puncture was performed without any delay (6). A final one-minute resting period concluded this session. The total duration of the control session was again approximately ninety minutes.

The order of the two sessions, like that of the tasks and the films, was fixed, with the stress session always coming first. The control session took place within one week after the stress session at exactly the same time of day. 
In section 2.2.1 it was shown that in this experiment the number of exhausted type A subjects and vital type B subjects was larger than that of vital type A subjects and exhausted type $B$ subjects. This means that when analyzing the effects of the grouping factors we are confronted with unequal cell sizes of the four subgroups.

There are two different solutions for this problem. One solution weighs for the number of subjects in each cell. This solution is appropriate when one is interested in the crude or uni-variate effects of each of the factors. The other solution gives equal importance to each of the cells, which allows the assessment of the independent contribution of the two grouping factors. We have chosen for the second solution since there is evidence that, not only in our sample but also in the population, the two risk factor are not independent. Van Doornen (1988) reported a correlation of 37 (van Doornen, 1988) between the Jas and the MQ scores, and Falger (1989) showed that type A subjects were significantly more exhausted than type B subjects. Given the association between the two risk factors the uni-variate assessment of their influence on reactivity in not appropriate when both are expected to have an effect on reactivity.

Analyses of variance were performed separately on all dependent variables using $A B$ typology and level of exhaustion as group factors, and experimental condition, task ws baseline, as a repeated measure (BMDP program $\mathrm{P} 2 \mathrm{~V}$ ).

Differences in reactivity between type A and B subjects or between Vital and Exhausted subjects were tested by inspecting the interaction between group and condition. Whenever significant interactions were found, the level of activation of high and low risk subjects was compared separately for the task and the baseline condition. This allowed us to check whether the significant interaction was indeed the result of equal baseline values and higher stress values in the high risk subjects.

\section{RESLLTS I: COMPARISON OF TASK SESSION AND CONTROL SESSION}

As a first step in the analysis mean values of mood and physiological variables were computed both for the task and for the film session, resulting in a stress and a control score respectively. These scores, together with the hormonal values and the platelet aggregation values obtained during the task session or the control session, were used to see to which extend the experimental tasks led to higher stress levels than the films. They were also used to test the overall hypothesis of difference in reactivity between type $\mathrm{A}$ and $\mathrm{B}$ subjects. Furthermore, differences between exhausted and vital subjects in this respect were examined.

\subsection{1}

Main condition effects

In table 2.3.1 mean scores of the dependent yariables obtained during the task and the control session are shown. These mean walues were computed by taking the mean of 
the four subgroup means instead of the overall mean. In this way the effect of unequal numbers in each subgroup was eliminated by giving equal weight to all four subgroups just like in the analysis of variance itself.

Subjective state. Significant differences in distress scores between control and task session were found indicating that the tasks were experienced as more distressing. Also the arnount of effort spent on the tasks was significantly higher than on watching the films.

Physiological activation. The differences in physiological activation between control and task session was generally in the expected direction Inter-beat-interval, pulse arrival time and respiration rate indicated higher activation during the task session. The higher amount of heart rate variability was surprising since usually increased activation leads to a suppression of variability.

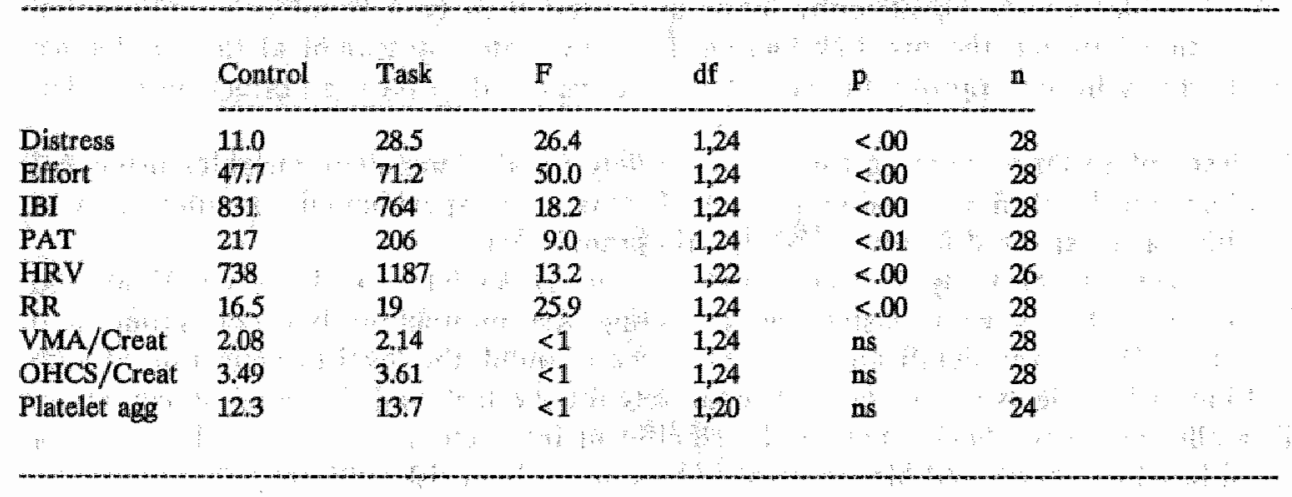

Table 2.3.1: Mean scores of the dependent warlables during the control and the task sesslon. (IBI is inter-beat-interval in milliseconds; PAT is pulse arrival time in milliseconds; HRV is heart rate variability with trequency of $8-12$ Hert expressed as $\%$ variation 10 ; $R R$ is respiration rate expressed in number of respiration cycles per minute; VMA/Creat is the ratlo of VMA and creatinine concentration. OHCS/Creat is the ratio of 17 - OHCS and creatinine; Platelet agg is the index of in vitro platelet aggregation the tangent of the steepest angle of the aggregation curve welghted by the amount of dilution of the trigger substance)

Hormonal excretion. Neither for the VMA/Creatinine ratio nor for the 17-OHCS/Creatinine ratio an overall difference between the task and the control session was found.

Platelet activity. No overall difference was found between the task and the control session.

\subsection{2}

Differences in reactivity between type $A$ and $B$ subjects

The mean values for type $A$ and $B$ subjects presented below are computed using the mean of the exhausted type A subjects and of the vital type A subjects and that of the exhausted type $B$ and the vital type $B$ subjects respectively. In this way the effect of 
unequal mumbers in each subgroup was eliminated by giving equal weight to each of the four subgroups like in the analysis of variance itself.

Subjective state. Neither for the distress scores nor for the effort scores a significant interaction between session and $A B$ typology was found. However a significant main effect of $A B$ typology was found $(F(1,24)=10.23 p<.004)$. Type $A$ subjects had a higher distress score than type $B$ subjects both during the task session and during the control session. In figure 2:3.1 the mean values are shown. No differences in change in effort were found between type A and B subjects, nor did they differ during any of the conditions.

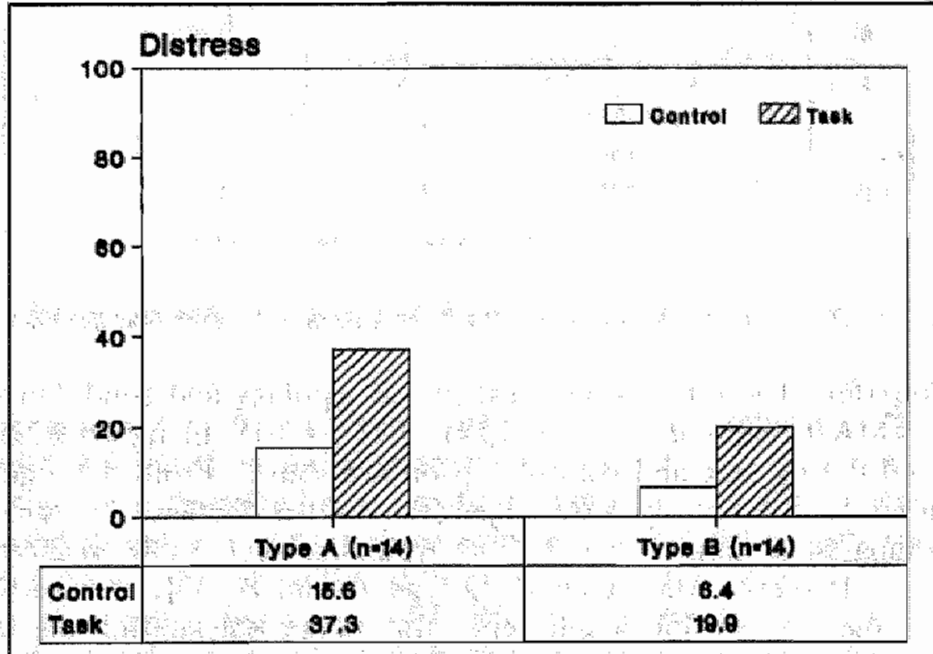

Figure 2.3.1. Dlstress scores from type $A$ and $B$ subjects during the tesk and the control sesslon.

Physiological activation. Neither for inter-beat-interval nor for pulse arrival time nor for respiration rate a significant interaction was found. Only for heart rate variability a significant interaction was found $(F(1,22)=6.91 \mathrm{p}<.02)$. From figure 2.3 .2 it can be seen that type A subjects show a smaller increase in heart rate variability than type B subjects. This is an unexpected finding since a larger decrease was predicted. 


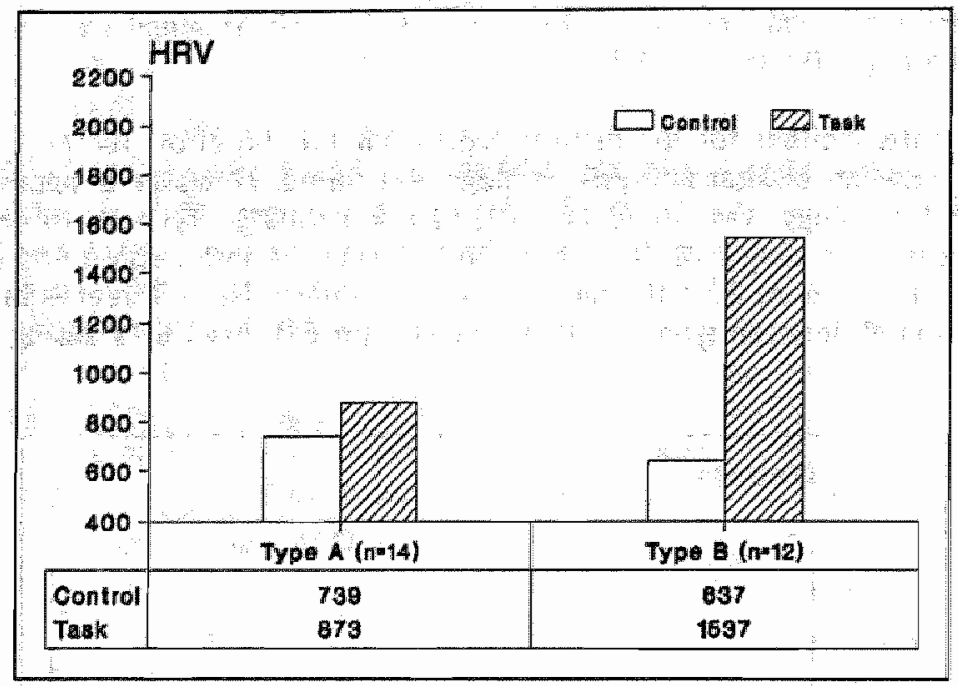

Figure 2.3.2: Mean HRV scores from type A and B subjects during the task and the control session.

Hormonal excretion. The interaction between $\mathrm{AB}$ typology and condition was significant for the VMA/Creatinine ratio $(F(1,24)=7.73 \mathrm{p}<.01)$. In figure 2.3 .3 the mean values obtained during task and control session are shown. From this figure one can see that, against expectation, type A subjects showed a decrease in ratio when task values are compared to control values while type B subjects show an increase during the task session. However when comparing type A and B subjects separately during the control or the task session a difference was found only during the task session $(F(1,24)=4.3 \mathrm{p}<.05)$. The interaction is, therefore, mainly due to a slightly lower value during the task in type A subjects. So in this experiment type A subjects did not show the expected greater reactivity, nor did they show a higher amount of VMA excretion during the control or the task session.

The interaction between $A B$ typology and condition for the 17-OHCS excretion was not significant. Again, type A subjects did not show a greater reactivity nor did they show a higher amount of 17-OHCS excretion during the control or the task session. 


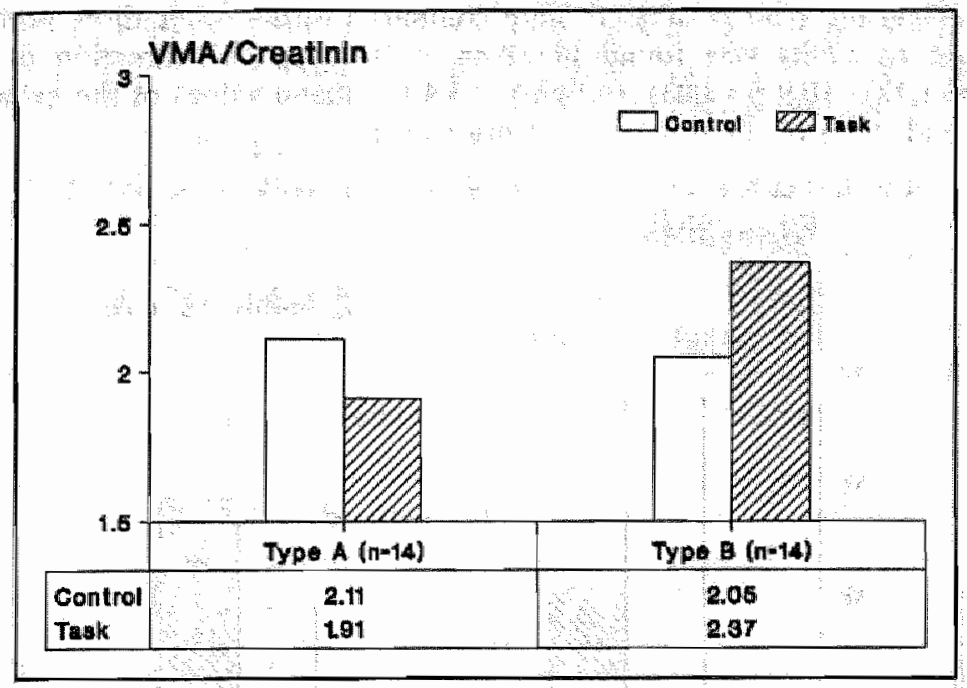

Figure 2.3.3: VMA/creatinhe values from type $A$ and $B$ subjects obteined during the task and the control sassion.

Platelet aggregation. No significant interaction was found between $A B$ typology and condition however, the third order interaction, although not to a significant degree $(F(1,20)=2.4 p<.14)$, suggested that the four subgroups did not change in a similar way when control and task session were compared. As can be seen from table 2.3.2 the exhausted type A subjects differed from the other three groups. Whereas most of the subjects did have a higher platelet activation score after the task session the exhausted type A subjects had a somewhat lower score after the task and a higher score after the film.

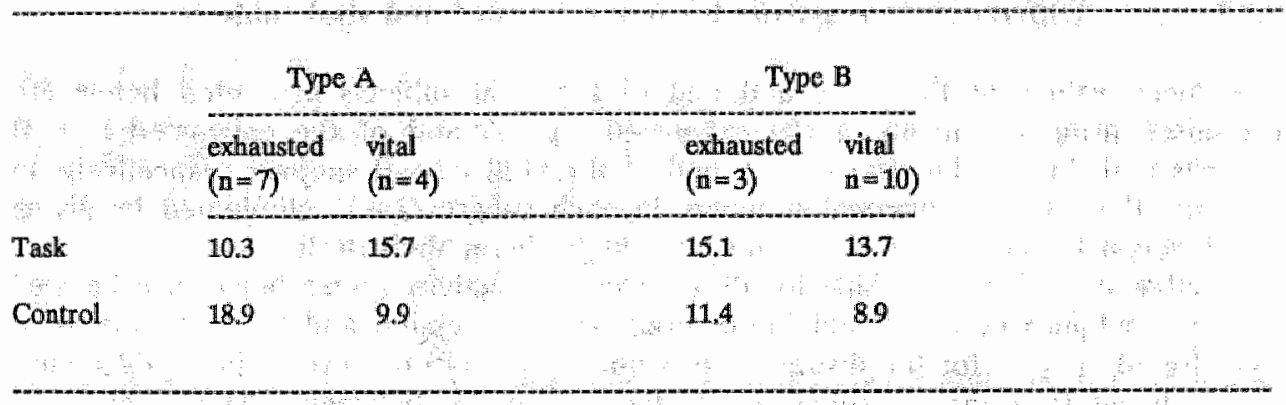

Table 23.2: Mean maximal aggregation scores of exhausted and vital typo A and exhausted and vftal type $B$ subjects after the task and the control session. 
When the exhausted type A subjects were compared to the other three groups a clear difference in reactivity was found however in the opposite direction of what was expected $(\mathrm{F}(1,22)=10.9 \mathrm{p}<.003)$. In figure 2.3 .4 the mean values of the exhausted type A subjects and those of the other groups are shown.

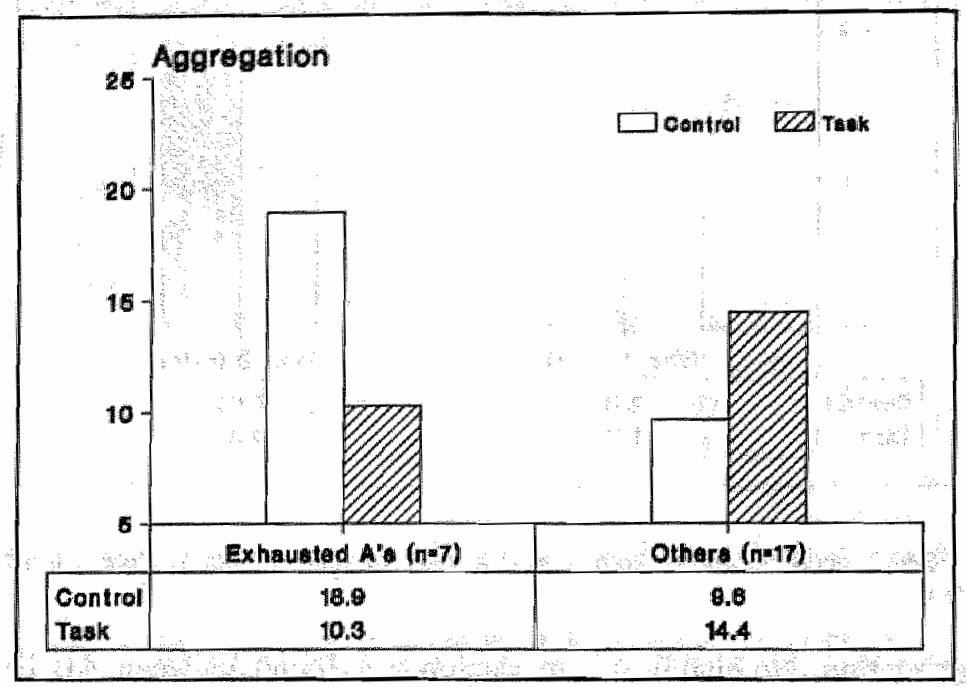

Figure 2.3.4. Mean maximal aggregation scores of exhausted type A subjects and mean scores of the other sublects after the task and the control session.

When compared separately after the task and the control session a significant higher aggregation score was found after the control session $(F(1,22)=9.3 \mathrm{p}<.005)$ but after the task session no differences were found.

\subsubsection{Differences in reactivity between exhausted and vital subjects}

The mean values of the exhausted and of the vital subjects presented below are computed using the mean of the exhausted type $A$ and of the exhausted type $B$ subjects and that of the vital type $A$ and of the vital type B subjects respectively. In this way the effect of unequal numbers in each subgroup was eliminated by giving equal weight to each of the subgroups just like in the analysis itself.

Subjective state. When looking for differences in reactivity scores between exhausted and vital subjects no significant interactions between session and level of exhaustion were found, neither for the distress scores nor for the effort scores. Thus a difference in the change from the control session to the task session between exhausted and vital subjects in self reported mood is not supported by these data. Moreover no overall differences between exhausted and vital subjects were found when they were compared during the task and the control session. 
Physiological activation. Only for heart rate variability a significant interaction between level of exhaustion and session was found $(F(1,22)=5.48 \quad p<.03)$. Separate comparisons of vital and exhausted subjects during the control and the task session did not reveal any significant difference.

Hormonal excretion. The interaction between level of exhaustion and condition on VMA/Creatinine ratio was not significant. The test of the main effect of vitality did not reveal a difference either so no difference in reactivity or in the amount of VMA excretion between vital and exhausted subjects could be established. A significant. interaction between vital exhaustion and condition was found for the 17-OHCS/Creatinine ratio $(F(1,24)=7,2 p<.01)$. Mean values of this ratio for exhausted and vital subjects obtained during the task and the control session are shown in figure 2.3.5.

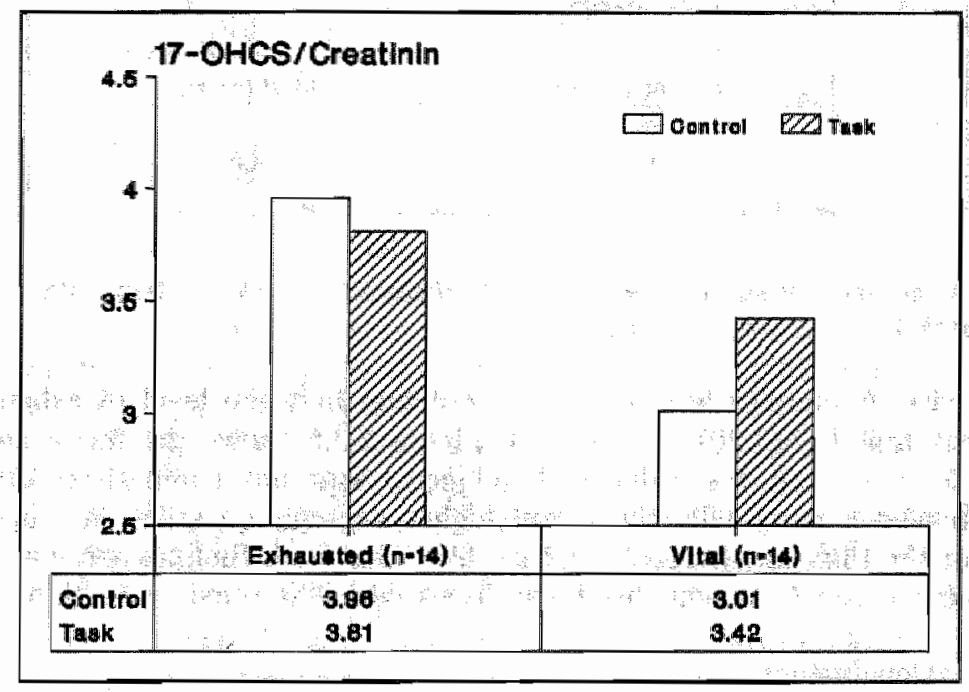

Figure 2.3.5: 17-OHCS/creatinhe ratios from exhausted and vital subjects obtalned during the task and the control session.

From this figure one can see that the exhausted subjects show a decrease during the task when these values are compared with the control values while the vital subjects show an increase when the same comparison was made. When vital and exhausted subjects were compared separately during the task and the control session, a significantly higher ratio was found in the exhausted subjects during the control session $(\mathbb{F}(1,24)=5.8 \quad p<.02)$. During the task, no differences emerged. The difference in reactivity is, therefore, due to a difference in baseline values and not to a difference in stress values. 


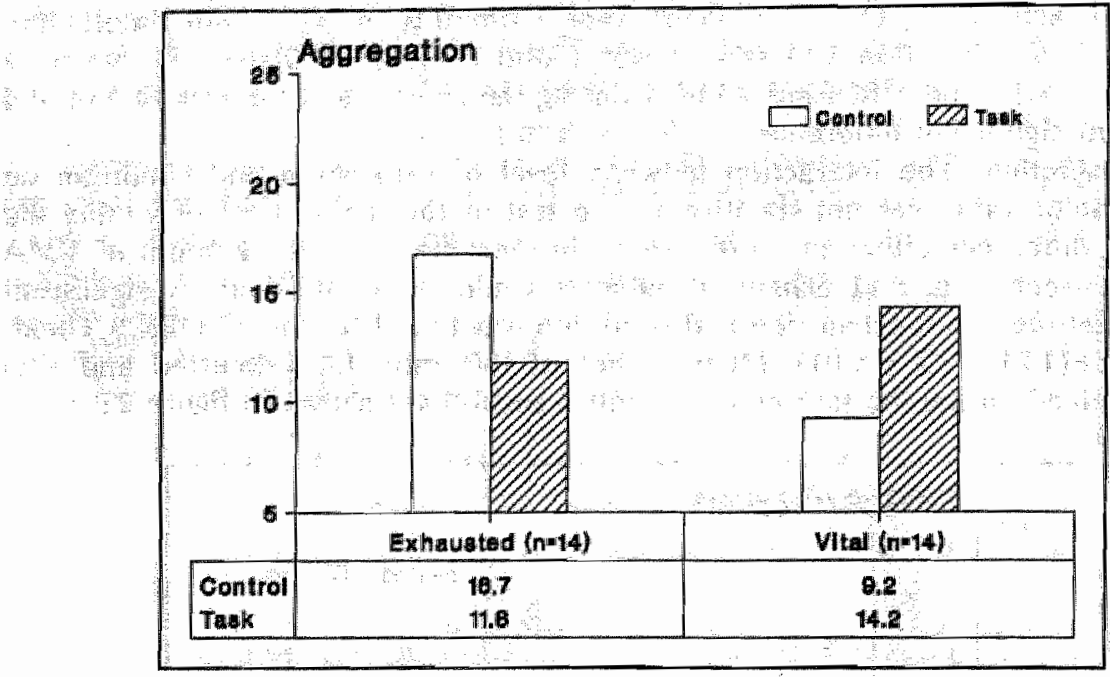

Figure 2.3.6: Mean maximal aggregation scores of exhausted and vital subjects after the task and the control session.

Platelet activity. A marginally significant interaction between level of exhaustion and condition was found $(F(1,20)=3.3 \quad p<.09)$. Figure 2.3.6 shows the mean aggregation scores of the vital and the exhausted subjects. Separate comparison under each condition showed a marginally significant higher aggregation score in the exhausted subjects after the film only $(F(1,20)=3.2 \mathrm{p}<.09)$. That both findings are mainly caused by the exhausted type $A$ subjects has been discussed in the previous section.

\section{3 .4}

Conclusions

Main condition effects. In this experiment the subjects did report more distress and more effort during the tasks than during the films although it should be noted that the degree of distress was not very high: only about one third of the amount the subjects had ever experienced.

Also the difference in physiological activation was in the expected direction. An explanation for the unexpected higher amount of HRV during the task session might by that during part of this session the subjects were speaking. Recent observations of Mulder (1988) have shown that during speaking tasks an increase in variability will be found, so the inclusion of the speaking task might well be the reason for the larger variability during the task session. When the analyses were repeated without the heart rate variability scores obtained during the quiz no interactions were found. This point will be addressed further when the separate analyses of the tasks will be discussed.

The absence of an overall difference in hormonal excretion and platelet activity between the task and the control session contrast sharply with the fairly consistent differences that were found between the two conditions on the subjective state 
variables and the physiological variables. Higher hormone ratios during the control session was found as often as higher hormone ratios during the task session whereas on the state variables and on the physiological variables the majority of the subjects had the highest values in the task condition. The same could be observed regarding the platelet activity: high and low aggregation sensitivity was found equally often after the task and the control session. The absence of a significant main effect in hormonal excretion and platelet activity could have been caused by opposing changes between the two sessions in the four subgroups. This will be further discussed in the following paragraph.

Global differences in reactivity: type $A$ vs type B. Consistent differences in reactivity between type A and B subjects were not found.

Although the type A subjects differed somewhat more clearly from type B subjects in their distress score during the task, the effect was not large enough to result in a significant interaction.

Also on the physiological variables no clearly interpretable differences were found that supported the main hypothesis.

Regarding the VMA excretion type A subjects had a higher excretion during the control session and a somewhat lower excretion during the task session than type B subjects which is the opposite of what was predicted, and on the 17-OHCS excretion no difference was found.

The absence of overall differences in platelet activity between the control and the task session might be explained by the difference between the exhausted type $\mathrm{A}$ subjects, who had the most sensitive platelets after the control session, and the other subjects who, as was expected, showed a higher sensitivity after the task session.

What could be the reason for the deviant pattern in the exhausted type A subjects? It may be that the exhausted type $A$ subjects experienced the control session as the more stressful condition and the other subjects the task session as more stressful. In support of this explanation is the finding that a reanalysis of the VMA/Creatinine excretion ratio during the task and the control session, using only those subjects that were used in the test for platelet aggregation, revealed a similar pattern as with the platelet aggregation data. A marginally significant interaction between group and condition was found $(F(1,22)=2.9 p<.10)$. In figure 2.3 .7 the mean ratios fo the two groups are shown. The exhausted type A subjects had a higher ratio during the control session while the other subjects had their highest ratio during the task session.

However when the two groups were compared separately after the task and the control session no significant differences were found. This means that this interaction can not be mainly explained by the higher VMA excretion in the exhausted type $A$ subjects during the control session. The opposite trend during the task session, 1.e. a lower VMA excretion rate in the exhausted type A subjects, has contributed to the interaction too.

A second point that would seem to support the explanation of higher distress during the control session was the amount of self reported distress. The distress scores of the exhausted type A subjects were significantly higher than those of the other subjects during the control session $(20.4$ vs 8.4$)(F(1,22)=8.0 \mathrm{p}<.01)$ and no differences were found during in the amount of distress during the tasks between the two groups. 


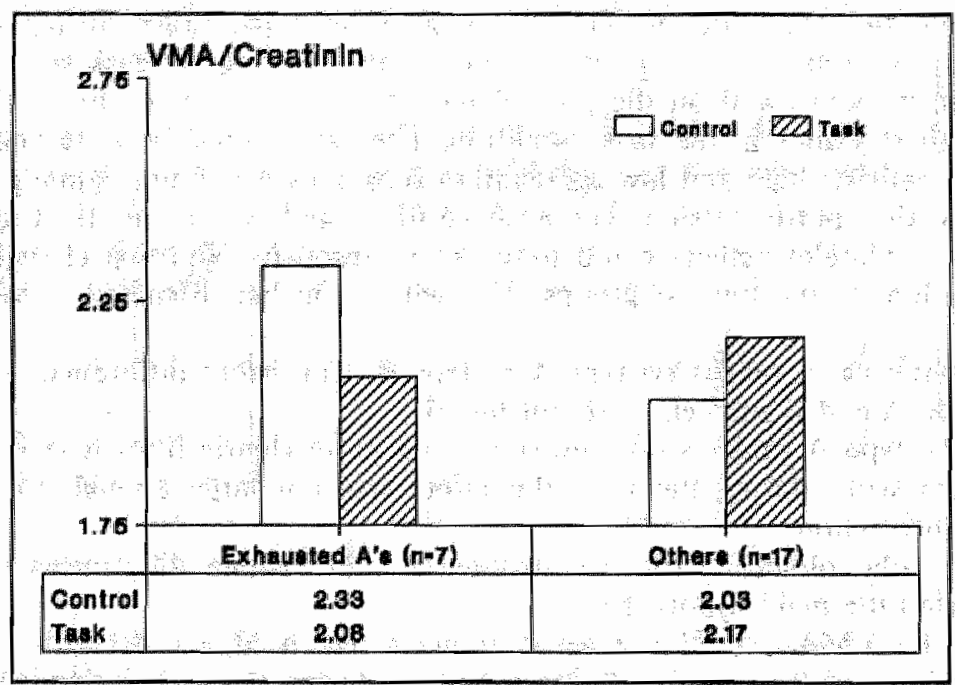

Figure 2.3.7: Mean WMA/Creatinine ratio from exhaustod type A subjects and from the other subjects during the task and the contral session.

On the other hand, both groups reported a higher level of distress during the task session than during the control session so the amount of distress in the exhausted type A subjects was not lower during the tasks than during the control session. Moreover none of the other variables did indicate higher activation during the control session than during the task session in the exhausted type A subjects so this explanation does not seems very probable.

It could also be that the exhausted type A subjects have platelets that are already more sensitive during baseline conditions than those of the other subjects. During the task session an increase in stress might have caused the platelets to be already so sensitized while they were still in the circulation that they might have formed microaggregates which either got stuck in the micro vessels or were extracted during the preparation of the platelet rich plasma leading to a test sample that contained either fewer platelets or only those that were less sensitive. This might have resulted in higher aggregation thresholds.

Global differences in reactivity: exhausted vs vital. No clear differences in reactivity between vital and exhausted subjects could be observed, neither in the subjective state, nor in the physiological activation. Rather puzzling is the absence of an overall difference in distress between the exhausted and the vital subjects. It might be possible that a retrospective judgement regarding psychological functioning and the actual state of psychological functioning are not very closely related, or that the selection criteria used to distinguish exhausted from vital subjects were not strict enough.

Regarding the hormonal excretion, against expectation, only during the control session 
a higher excretion of 17-OHCS was found. No differences between vital and exhausted subjects were found in VMA excretion.

The interaction found for platelet activity also was mainly caused by a higher aggregation tendency in the exhausted subjects during baseline. This effect was, in turn, mainly due to the exhausted type A subjects.

Using these overall scores no support was found for the main hypothesis of larger reactivity in type A subjects nor were there any consistent differences in reactivity between vital and exhausted subjects. Although these first analyses did not yield evidence for larger reactivity in high risk subjects one must realize that they were based on mean session scores averaged across tasks. It might be possible that the high risk subjects did react to some tasks with an larger increase in activation but not to all. Averaging could have obscured the differences. Therefore, in the next sections differences in reactivity will be examined in more detail by looking at the separate tasks.

As described in section 2.2.2 time pressure was manipulated by imposing different time limits within which a reaction had to be made during a dual choice reaction task. This enabled us to test the second hypothesis: the difference in physiological activation between type $A$ and $B$ subjects can be seen most clearly at a medium level of time pressure.

Regarding the effects of time pressure on exhausted subjects, no specific hypothesis was formulated; differences between vital and exhausted subjected were examined during the different time limits.

Complete data from 27 subjects were available on the performance variables. Analyses of variance were performed separately on reaction time and on percentage correct reactions of the first run of the reaction task, using $A B$ typology and vital exhaustion as group factors and time limit (with five levels) as repeated factor. For the physiological data complete data of 28 subjects were available. Analyses of variance were performed separately on IBI, PAT, HRV (medium frequency) and RR, on data obtained during the first run of the task with a pre-task resting period as covariate, $\mathrm{AB}$ typology and level of exhaustion as group factors and time limit (with five level) as repeated factor. The hypothesis was tested by looking at the interaction between $A B$ typology and time limits. Also the main effects of time limits were tested to see whether they did affect the way in which the subjects reacted.

Performance variables. On reaction time, a main effect of time limits was found to be significant $(F(92,4)=135.6 \mathrm{p}<.00001)$ indicating that reaction time decreased when the time to react was shortened. Also on percentage correct responses a main effect of time limits was found $(\mathrm{F}=135.6 \mathrm{df}=92,4 \mathrm{p}<.0001)$ indicating a decrease in the percentage of correct reactions as time limits became shorter. The mean values of 
both variables are shown in figure 2.4.1

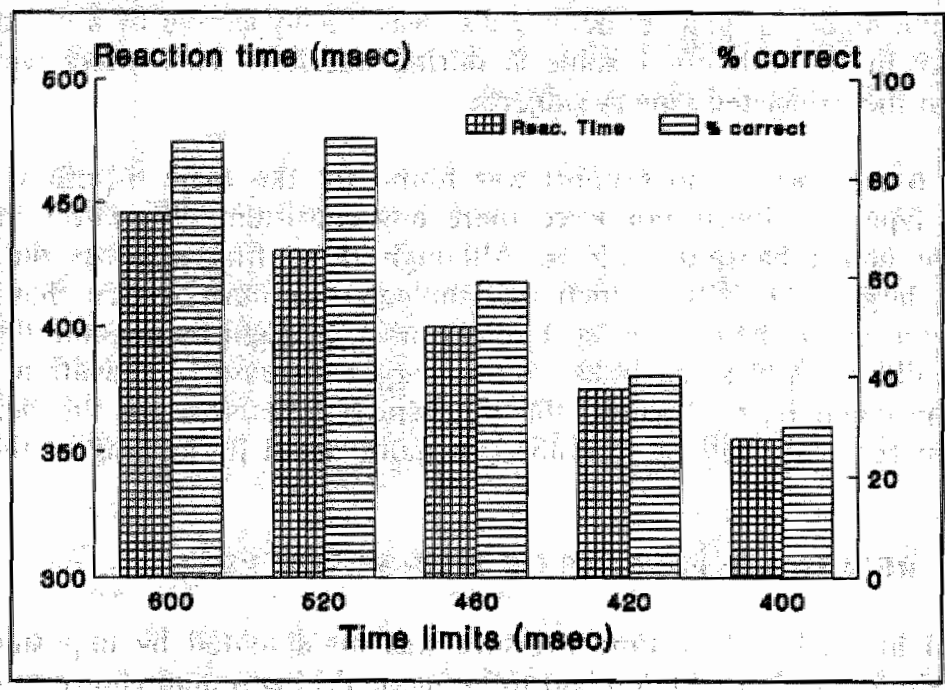

Figure 2.4. Thean reaction time and percentage correct responses during the different time lim/ts.

Physiological variables. No effect of time limits was found for IBI, PAT, HRV or RR.

\section{4 .2}

\section{Differences between type A and B subjects}

Performance variables, Neither on RT nor on percentage correct reactions interactions between time limits and $A B$ typology were found, nor were there any overall differences between the two groups during the execution of the task.

Physiological variables, On none of the variables an interaction or an overall difference during the reaction task was found.

\subsection{3}

\section{Differences between exhausted and vital subjects}

Performance variables. An interaction between level of exhaustion and time limits was found for the percentage correct reactions $(F=(92,4)=4.33 \mathrm{p}<.003)$. From figure 2.4 .2 it can be seen that the exhausted subjects made less errors than the vital subjects at low levels of time pressure.

During medium levels the scores are similar, while during the highest level of time pressure the exhausted subjects made somewhat more errors than the vital subjects. On the reaction time no interaction with vital exhaustion was found nor was there an overall difference between the exhausted and the vital subgroups.

Physiological variables No interactions nor any overall differences were found on any of the variables. 


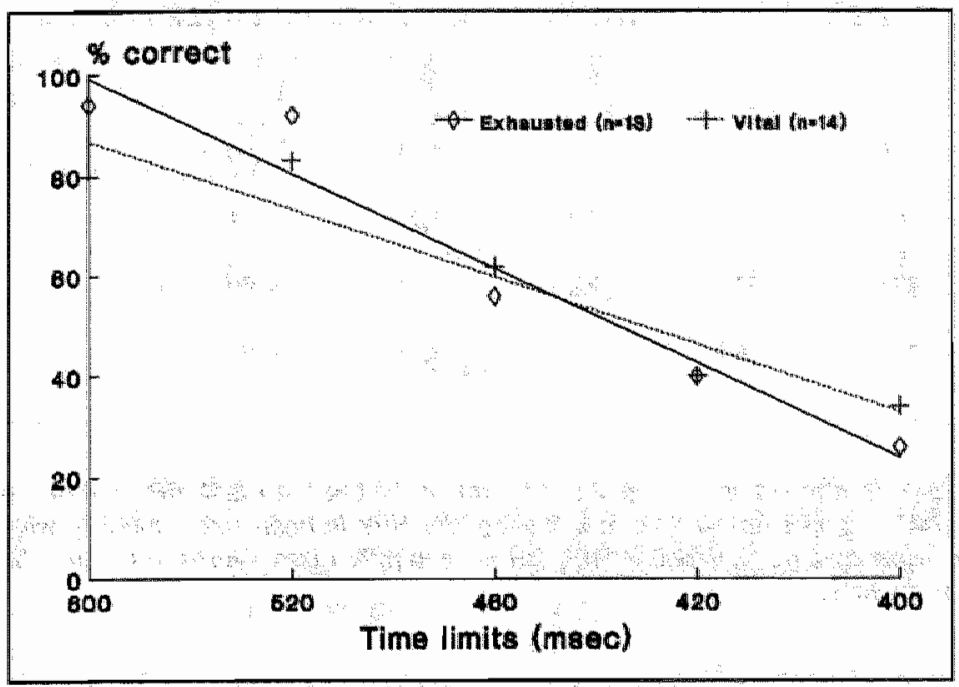

Flgure 2.4.2. Mean percentage correct responses in exhausted and vital subjects during five different time limits.

\subsection{4}

Conclusions

The results of this experiment indicate clearly that performance is influenced by imposing different time limits on the subjects. The subjects reacted faster and made fewer correct reactions when the time limits became more strict.

An interesting observation was the different way in which the performance of the vital and the exhausted subjects was affected by the time limits. The exhausted subjects reacted more accurately than the vital subjects at low time pressure but they could not keep up their better performance as time pressure increased and it even got worse than that of the vital subjects when time pressure became high.

No evidence was found of a different effect of time pressure on the performance of type A subjects. Making the time limits shorter did not affect the physiological variables in any of the subgroups.

To check whether the performance of the task itself did result in a change in physiological activation a comparison was made between the task minutes and the resting minutes following each task minute. From table 2.4 .1 it is clear that during the task performance all physiological variables indicated an increase in activation. Since there where no interactions between tasks vs no-task periods and either $\mathrm{AB}$ typology or vital exhaustion, we may conclude that performing a reaction task did lead to a uniform increase in physiological activation in all subgroups that was not affected by the different time limits.

This means that no evidence was found for the hypothesis tested in this section. 


\begin{tabular}{l|ccccc|c|c|}
\hline & & task & rest & F & df & p & \\
IBI & 738 & 758 & 18.3 & 1,24 & $<.0003$ & \\
PAT & 201 & 204 & 7.9 & 1,24 & $<.01$ & \\
HRV & 374 & 978 & 9.8 & 1,24 & $<.005$ & \\
RR & 23.1 & 16.2 & 114.6 & 1,24 & $<.0000$ & \\
\hline
\end{tabular}

Table 2.4. \%: Task vs rest values during the first run of the reaction task (IBI is inter-beat-interval in miliseconds, PAT is pulse arrival time in milliseconds; HAV is heart rate variability with a frequency of 8-12 Hertz expressed as \% variation*102; RR is respiration rate expressed in number of respirathon cycles per minute).

\section{5}

\section{RESULTS III: EFFECTS OF CRITICISM}

As described in section 2.2 .2 the subjects were either praised or criticized after they had completed the first reaction task. The same was done during the quiz. In this section the third hypothesis was tested: the difference in subjective and physiological activation between type $A$ and $B$ subjects is seen more clearly after criticism than before feedback is given. Differences between vital and exhausted subjects in this respect were also examined, like differences in performance between the two types of subgroups.

Since the reaction to criticism can be either the mobilization of effort to perform better or a giving up reaction leading to a lower investment of effort it is not possible to predict what the general effect of criticism will be. The same hold for the effects of praise. Subjects can loose interest in the task, which they evidently have mastered, and lower their effort or stay at the same level of investment, or they may be encouraged to work even harder since they seem to be good at the task. Thus, no specific predictions were made regarding the main effects of feedback.

Two tests of the hypothesis were available: one involving the reaction task and one involving the quiz.

\subsubsection{Feedback on performance after a reaction task}

Data from 28 subjects were available for statistical analysis of the physiological and the mood variables. Performance data of one type B subject in the negative feedback condition were missing. Separate analyses of covariance were performed on each dependent variable using either $A B$ typology or vital exhaustion and type of feedback as grouping factors since the number of subjects did not allow the simultaneous use of all three grouping factors. As covariates the values obtained during the first run of reaction task were used in order to minimize the within group variability. The interactions between feedback and either $A B$ typology or level of exhaustion were 
tested to see whether positive and negative feedback led to different reactions. Subsequently high and low risk subjects were compared separately after positive and after negative feedback.

\subsubsection{Main effects of feedback}

On none of the dependent variables an overall difference between those subjects who received positive feedback and those who received negative feedback could be found.

\subsubsection{Differences between Type A and B subjects}

In table 2.5 .1 the mean values of the dependent variables from type A and B subjects, corrected for pre-feedback levels, after criticism or praise are presented.

Performance variables. No interaction between AB typology and type of feedback was found for reaction time. Also the reaction time of type A did not differ from that of type B subjects after either positive or negative feedback.

Regarding the percentage correct responses an interaction was found between $\mathrm{AB}$ typology and type of feedback $(F(1,22)=3.1 p<.09)$. From table 2.5 .1 it can be seen that the interaction is explained by the tendency in type $A$ subjects to react with more correct responses after positive feedback while type B subjects tended to reacted more often correctly after negative feedback. However these differences were not large enough to result in significant differences when type A and B subjects were compared separately after positive and after negative feedback.

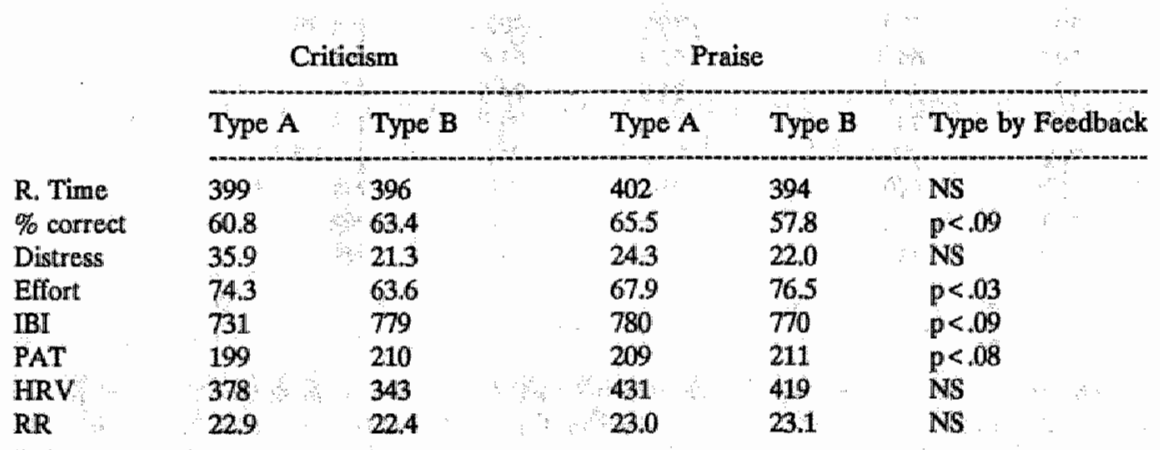

Table 2.5.1: Mean values of the dependent varlables from type $A$ and $B$ subjects corrected for prefeedback values, after positive and negative feedback on their performance of a reaction task. (R.Time is reaction time in miliseconds; \% correct is percentage correct responses; IBI /s interbeat-Interval in miliseconds; PAT is pulse arrival time in milliseconds; HAV is heart vate varlability with a frequency of B-12 Hert expressed as \% variation*10\%, RA ls resplration rate expressed in number of respiration cycles per minute). 
Subjective state. $A$ significant interaction between $A B$ typology and type of feedback was found for the amount of effort spent on the task $(F(1,23)=5.9 \mathrm{p}<.03)$. From table 2.5 .1 it can be seen that the interaction is caused by the fact that type A subjects tended to increase their effort after negative feedback while type $B$ subjects did the same after positive feedback.

Howewer a comparison between type A and B subjects did not result in a sigaificant difference neither after positive nor after negative feedtback, so the above noticed differences between type $A$ and $B$ subjects. can only be regarded as marginal irends.

No dear interaction between $A B$ typology and feedback could be found for distress, but after megative feedlback type A subjects had a higher distress score than type B subjects. $(F(1,11)=3.4 p<.09)$.

Physilological variables. Significant interactions between $A B$ typology and type of feedback were found for IBI $(\mathrm{F}(1,23)=3.2 \mathrm{p}<.09)$ and $\mathrm{PAT}(\mathrm{F}(1,23)=3.4 \mathrm{p}<.08)$. For HRV and RR no interactions were found. When type $A$ and $B$ subjeets were compared after negative feedback differences were found for IBI $(F(1,11)=4,0 \quad p<.07)$ indicating shorter IBI's in type A subjects than in type B subjects and for PAT $(F(1,11)=48 \mathrm{p}<, 05)$ also indicating shorter PAT's in type A subjects than in type B subjects. After positive feedback no differences were found.

\subsubsection{Differences between exhausted and vital subjects}

In table 2.5 .2 the mean values of the dependent variables from exhausted and vital subjects, corrected for pre-feedback levis, after criticism or praise are given.

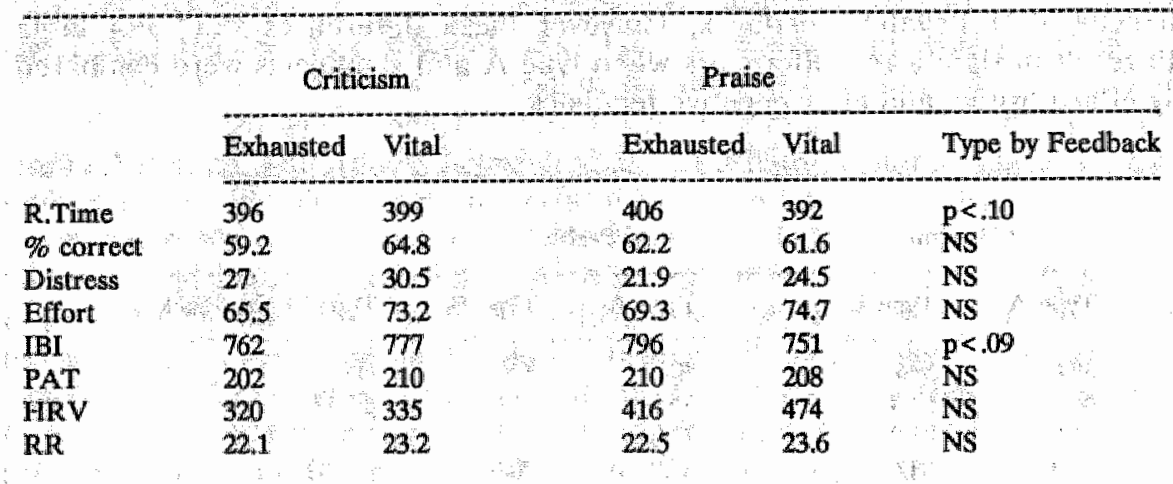

Table 2.5.2. Mean values of the dependent varlables from exhausted and vital subjects, corrected for pre-feedback values, after positive and negative feodback (IBI is inter-beat-interval in milliseconds; PAT is pulse artival time in millisecionds; HRV is heart rate variablity with a frequency of 8-12 Hertz expressed as \% varlation"10; RR is respiration rate expressed in number of respiration cycles per minute).

Performance variables. Only a marginal interaction between level of exhaustion and type of feedback on reaction time was found $(F(1,22)=3.0 \quad p<10)$. After positive feedback the vital subjects reacted faster than the exhausted subjects $(F(1.11)=4.2$ $\mathrm{p}<.07)$. When exhausted and vital subjects were compared after negative feedback no difference in reaction time was found. The exhausted subjects did have a lower percentage of correct responses $(\mathrm{F}(1,11)=6.45 \mathrm{p}<.03)$ after they were criticized. 
Subjective state. No interactions between level of exhaustion and type of feedback were found. The overall comparison between vital and exhausted subjects did not result in a significant difference either.

Physiological variables. A significant interaction between level of exhaustion and type of feedback was found for IBI only $(F(1,23)=3.1 p<.09)$. Inspection of the mean IBI values of the four subgroups showed that after negative feedback the exhausted group had somewhat shorter IBI's than the vital group. After positive feedback the vital subjects had the shorter IBPs. Separate comparisons revealed that only after positive feedback a significant difference between exhausted and vital subjects could be demonstrated $(\mathbb{F}(1,11)=3.9 \mathrm{p}<.08)$.

\subsubsection{Feedback on performance during the quiz}

Data from 28 subjects were available for statistical analysis. The same type of analysis of covariance was used as in the previous section. As covariates values obtained during a resting period before the first reaction task were used. The interactions between the two grouping factors were tested to see whether positive and negative feedback led to different reactions. Subsequently high and low risk subjects were compared separately in the positive and the negative feedback condition.

\subsubsection{Main effects of feedback}

On none of the dependent variables an overall difference between those subjects who received positive feedback and those who received negative feedback could be found.

\subsubsection{Differences between type $A$ and $B$ subjects}

In table 2.5.3 the mean values of the dependent variables during the quiz from type $\mathrm{A}$ and B subjects, corrected for pre-feedback levels, are given.

Performance variables. No interaction between feedback and $A B$ typology was found nor did type $A$ and $B$ subjects differ in general regarding the percentage correctly answered questions.

Subjective state variables. $\mathrm{A}$ significant interaction between $\mathrm{AB}$ typology and type of feedback was found, both for effort $(F(1,23)=3.3 p<.08)$ and for distress $(F(1,23)=4.1$ $\mathrm{p}<.05$ ). Separate comparisons between type $A$ and $B$ subjects revealed that distress was higher in type $A$ subjects than in type $B$ subjects during negative feedback $(\mathrm{F}(1,11)=3.2 \mathrm{p}<.10)$ while effort was lower in type A subjects than in type B subjects during positive feedback $(F(1,11)=3.9 \mathrm{p}<.07)$.

Physiological variables. $A$ significant interaction between $A B$ typology and type of feedback was found for PAT $(F(1,23)=5.1 \mathrm{p}<.03)$ No interaction but a main effect of $A B$ typology was found for $H R V(F(1,23)=4.4 p<.05)$ indicating more suppression of HRV in type A subjects than in type B subjects. A comparison of type A and B subjects during negative feedback revealed significant differences in PAT $(F(1,11)=3.7$ $p<.08)$ and in HRV $(F(1,11)=3.5 p<.09)$. Type A subjects had a shorter PAT and more suppression of HRV. During positive feedback no differences were found. 
Criticism

Praise

\begin{tabular}{|c|c|c|c|c|c|}
\hline$\because:$ & Type A & Type B & Type A & Type B & Type by Feedback \\
\hline \% correct & 56.7 & 622 & 63.7 & 623 & $\mathrm{Ns}$ \\
\hline Distress & 379 & 210 & 16.6 & 25.5 & $\mathrm{p}<-05$ \\
\hline Effort & 71,4 & 69.1 & 58.6 & 776 & $p<08$ \\
\hline IBI & 778 & 830 & 764 & 728 & $\mathrm{p}<11$ \\
\hline PAT & 202 & 217 & 212 & 202 & $\mathrm{p}<, 03$ \\
\hline HIR & 106 & 251 & 156 & 203 & NS \\
\hline $\mathrm{RR}$ & 16.4 & $\mathbb{1 5 5}$ & 15.5 & 16.9 & NS \\
\hline
\end{tabular}

Table 2.6.3: Mean values of the dependent variables from type $A$ and $B$ subjects, corrected for prefeodback values, during the quiz whille being crilicized or preised (IBI is inter-beat-intenval in miliseconds; PAT ls pulse arrival time in milliseconds; HAV is heart rate varlabillty with a frequency of 8-12 Hertz expressed as \% variation* $10^{2}$. FR is respiration rate expressed in number of respiraton cycles per minute).

\subsubsection{Differences between exhausted and vital subjects}

In table 2.5.4 the mean values of the dependent variables during the quiz from exhausted and vital subjects, corrected for pre-feedback levels, are given.

Criticism

\begin{tabular}{llllll}
\hline Exhausted & Vital & Exhausted & Vital & Type by Feedback \\
\hline 56.3 & 63.6 & 60.4 & 650 & NS & \\
28.0 & 30.0 & 11.1 & 29.6 & NS \\
69.9 & 74.8 & 59.0 & 74.8 & NS \\
812 & 798 & 780 & 716 & NS \\
205 & 215 & 212 & 203 & NS \\
166 & 193 & 198 & 167 & NS \\
16.1 & 15.7 & 15.1 & 17.0 & NS
\end{tabular}

Table 2.5.4: Mean values of the dependent varlables from exhausted and vital subjects during the quiz, corrected for pre-feedback values, while being crtticlized or preised (le) is inter-beat-interval in milliseconds; PAT is pulse arrival timo in miliseconds; HRV is heart rate variability with a frequency of 8-12 Hertz expressed as \% variation*10\% RR is respiration rate expressed in number of resplration cycles per minute).

Performance variables. Exhausted and vital subjects did not differ in the percentage correctly answered questions neither during positive nor during negative feedback. 
Subjective state variables. No interactions between level of exhaustion and type of feedback were found. Only during positive feedback did the exhausted subjects report less distress than the vital subjects $(F(1,11)=10.5 p<.01)$.

Physiological variables. No interactions between level of exhaustion and type of feedback were found on any of the four physiological variables. Only during positive feedback did the exhausted subjects have a longer mean IBI than the vital subjects $(\mathrm{F}(1,11)=3.1 \mathrm{p}<.09)$.

\subsubsection{Conclusions}

Type A vs type B subjects. When type A and B subjects were compared under condi tions of positive and of negative feedback significant differences were only found under negative feedback conditions.

After they were told that their performance was far below expectation, type A subjects had shorter inter beat intervals and shorter pulse arrival times during the reaction task, they reported more distress and showed a tendency to invest more effort but non the less made fewer correct response than type B subjects. Taking into account that the two types did not differ during the first reaction task nor under the positive feedback condition, the results of the reaction time experiment are generally in agreement with the saliency hypothesis tested in this section.

During the quiz the findings were in the same direction but of a lesser magnitude. Although type A subjects still had shorter inter-beat-intervals than type B subjects during negative feedback, the difference failed to reach significance. The difference in pulse arrival times and heart rate variability were also only marginally significant. In this part of the experiment again no differences between type A and B subjects were found during positive feedback.

It should be noted that in the statistical analysis used to test the hypothesis about the effect of criticism AB typology and vital exhausted were not used simultaneously. This means that in this analysis only the crude effects of each of the risk factors has been tested. The risk that the positive findings with respect to $A B$ typology are confounded by the over-representation of exhausted subjects in the type A group seems small, since the observed effect of a larger difference between type A and B subjects after criticism was not found when the exhausted and the vital subjects were compared after criticism.

Taking the results of the two tasks together we conclude that under the condition of negative feedback the differences between type A and B subjects were indeed more prominent even if they were still rather small.

Exhausted vs vital subjects. The explorative analyses of the physiological and performance characteristics of exhausted subjects showed a decrease in heart rate after positive feedback, both in the reaction task and during the quiz. Negative feedback did not result in marked physiological responses but decrease in percentage correct reactions in the second run of the reaction task showed that criticism resulted in a performance decrement. This was not replicated during the quiz however.

No difference was found between exhausted and vital subjects in the way their subjective state was affected. 
Given the incidental character of these findings we hesitate to draw any conclusions before further replication has been obtained.

On a number of occasions during the first two experiments the subjects were waiting between tasks or waiting before and while a venous puncture was done. These conditions have in common that the subject has to sit passively till the situation changes. It was expected that this would be particularly unpleasant for type A subjects since it would deprive them from their normal way of coping: to be actively engaged in resolving an unwanted situation. In this section the fourth hypothesis: oype $A$ subjects show higher levels of physiological activation than type B subjects during sifuations in which active coping is inappropriate, was- tested. Furthermore, differences between vital and exhausted subjects were examined under these conditions.

\subsection{1}

Resting periods during the first run of the reaction task

Physiological data from 28 subjects were available. Since no separate ratings of the resting periods were made, no mood scores could be analyzed. Separate analyses of covariance were performed on the mean scores of the five resting periods for IBI, PAT, HRV and RR, using AB typology and level of exhaustion as grouping factors with the pre-trial resting values as a covariate.

No main group factor nor the interaction between the group factors was significant.

Resting periods during the second run of the reaction task

Again physiological data from 28 subjects were available. Because the subjects had received feedback this factor was used as a grouping factor in the analyses of covariance. Since the number of subjects was too small to use three grouping factors either AB typology or level of exhaustion was used together with type of feedback as second grouping factor with the mean pre feedback resting values as a covariate.

Type A vs type B subjects. Significant interactions between AB typology and type of feedback were found for IBI $(F(1,23)=6.5 p<.02)$ and PAT $(F(1,23)=3.5 \quad p<.07)$. Separate testing of the differences between type A and B subjects after positive and negative feedback resulted in significant effects for IBI $(F(1,11)=5.3 p<.04)$ and PAT $(F(1,11)=3.6 \quad p<.08)$ only after negative feedback. During this condition type A subjects had both shorter mean IBI's and shorter mean PAT's (764 msec vs $809 \mathrm{msec}$ and 205 msec vs 214 msec respectively) than type B subjects. No differences were found for HRV and RR.

Exhausted vs vital subjects. A significant interaction between level of exhaustion and type of feedback was found for IBI $(F(1,23)=3.35$ p<.08). Separate testing of the differences between exhausted and vital subjects after positive and negative feedback resulted in a significant effect $(F(1,11)=5.3 \quad \mathrm{p}<.04)$ after positive feedback only. Longer mean IBI's were found in the exhausted subjects ( 852 vs $808 \mathrm{msec}$ ). No further 
differences between vital and exhausted subjects were found.

\section{6 .3}

Anticipation of venous puncture

Complete data from 28 subjects were available. Separate analyses of covariance were performed on the physiological variables using $A B$ typology and level of exhaustion as grouping variables with pre trial resting values as a covariate.

No main group effects nor an interaction between groups was found.

\subsection{4}

\section{Venous puncture}

The same analyses as in section 2.63 were performed and again no main effects of groups or an interaction between groups was found.

\section{6 .5}

\section{Conclusions}

Type A vs type B subjects. It was only after the subjects received negative feedback about their performance that some findings were obtained that were in agreement with the hypothesis tested in this section. Only under this condition was the level of physiological activation in type $A$ subjects relatively high compared to that of type $B$ subjects during the periods they could not work on the reaction task.

The fact that no differences were found before and during the venous puncture was at first surprising. However from the debriefing talks that were held with the subjects we learned that the majority of the subjects were quite familiar with the procedure of venous puncture since they were blood donors. Indeed the level of reported distress during this part of the experiment was very low and this might well explain why no differences were found.

From these results we conclude that it is necessary that type A subjects feel the urge to achieve something in order to show their higher activation level during passive coping conditions.

Exhausted vs vital subjects. No consistent differences in level of physiological activation between exhausted and vital subjects were found during passive coping conditions. The exhausted subjects had a lower mean inter beat interval after they received positive feedback on their performance on the reaction task. Given the incidental character of this finding we hesitate to draw any conclusions regarding the effect of passive coping conditions on exhausted subjects.

\section{7}

\section{MAIN CONCLUSIONS AND DISCUSSION}

The major findings of this experiment are the following:

1 The tasks did induce a general increase in subjective distress and in the amount of effort the subjects had to spent. 
2 During the tasks the physiological activation increased in all subjects.

3 No clear increase in platelet sensitivity or hormonal excretion could be found during the task session.

4. No support for the global hypothesis of greater reactivity in type A subjects was found when the mean scores of the task and the control session were compared.

5 Manipulation of time pressure did not affect the type A subjects in a different way than it did type B subjects.

6 When they were criticized type A subject did react more strongly than type B subjects with an increase in subjective arousal and physiological activation, both when working at the task and when waiting between task periods.

7 Particularly during the control session 17-OHCS excretion and platelet sensitivity was higher in the exhausted subjects than in the vital subjects.

8 The performance of the exhausted subjects on the reaction task became less accurate when time pressure increased. The same was found after they were criticized.

9 After positive feedback the heart rate of the exhausted subjects was low compared to that of the vital subjects, both during the tasks and in between the reaction task periods.

Absence of generally higher reactivity in type A subjects. In the previous sections not much evidence was found for the first hypothesis that type A subjects generally show a larger increase in physiological activation than type B subjects when confronted with a demanding task.

In the introductory chapter it was noticed that experiment in which short lasting tasks had been used differences in reactivity have been reported more often. An explanation for lack of difference in reactivity in this experiment might be that only during the initial part of the task the change in activation was larger in type A subjects but that during the course of the task their activation decreased to a level comparable to type B subjects. By using mean task scores these initial differences in reactivity might have be masked.

Therefore, a secondary analysis was performed using only the initial periods of the different tasks. These were both compared with the mean value of the first resting period of the control session and with the preceding resting period in the task session. No evidence supporting this explanation of the lack of differences between the subgroups was found since using these first minute comparisons did not result in any significantly larger response in type A subjects which had not already been found when mean task scores were used.

From these findings we conclude that there is no support for the global hypothesis of 
general larger reactivity in type $A$ subjects.

Infuence of task saliency. When the saliency of the task was higher a larger increase in activation was occasionally found.

Criticizing the performance of the subjects was the most effective way of increasing the sallency. Furthermore, when the subjects were temporarily prevented to work on a task on which they tried to improve themselves, also a larger reactivity was found in type A subjects.

Passive stressors in themselves were not effective in showing a larger reactivity in type A subjects.

Also ${ }_{*}$ increasing time pressure by shortening the time interval in which the subjects had to react did not lead to a clearer difference in reactivity. Since, because of practical limitations, no subjective state scores were obtained for each of the different time limits we cannot be sure whether subjective state was influenced by the different time limits. Moreover, there was no main effect of time pressure on the physiological variables. This, combined with the absence of an interaction between time pressure and $A B$ typology or vital exhaustion, strongly suggest that in none of the subgroups shorter time limits led to a change in physiological activation.

Differences between vital and exhausted subjects. Regarding the exhausted and vital subjects no indications of a difference in reactivity was found in the global analysis.

The higher excretion of 17-OHCS and the higher platelet sensitivity during the control session are not readily explainable and should be replicated before an explanation other than a chance finding is to be considered.

The finding that the exhausted subjects have a lower heart rate after praise and show a larger deterioration in accuracy when time pressure increases and when they have been criticized leads to the idea that exhausted subjects are less able to cope with highly demanding situations. This hypothesis should be tested in further experiments.

Lack of task effect in hormonal excretion and platelet aggregation. The explanation of the absence of an overall difference between task and control in hormonal excretion and platelet activity, as being due to opposing changes from task to control in the four subgroups, is not likely to be true since no differences in reactivity between the subgroups were found when mean session scores were used.

An other explanation of the absence of significant differences in hormonal excretion between the two sessions might be that, due to its cumulative character, the method of assessing hormonal activity using urine samples is less sensitive to changes in experimental condition than the self reported state variables and the physiological variables. The urine samples are collected during the total time of the session, including those parts that are the same in both sessions like preparation of the subject for the physiological measurements, instruction, resting periods and the taking of blood samples. These common elements take up to a third of the total session time thus making the contrast between the sessions less clear than when using only values obtained during the task periods or the film periods as has been done for the other two types of variables.

Regarding the lack of an overall difference between task and film values of platelet activity, the possible non-linear relation between the platelet sensitivity in viwo and the aggregation threshold, which was measured in vitro, might have played a role. 
Chapter 2

In order to avoid these difficulties, hormone arsessment should be more closely related to the tasks, and a method of assessing platelet activity that reflects the in vivo sensitivity is preferable to the in vitro aggregation test. 


\section{DIFFERENCES IN VIGILANCE BEHAVIOR BETWEEN HIGH AND LOW RISK SUBJECTS}

In the experiment that is described in this chapter subjects were confronted with monotonous vigilance tasks to explore possible differences in reactivity between type $A$ and type B subjects during mental understimulation. This was done to further explore which experimental situations were suitable to trigger core characteristics of type A behavior. In section 3.1 the hypotheses are stated, in section 3.2 the experiment is described in detail, in section 3.3 and 3.4 the results are presented and in section 3.5 the results are summarized and discussed.

\section{1}

\section{HYPOTHESES}

Vigilance tasks require continuous attention and the ability to wait for long periods of time without making a response. There is some evidence that type A subjects do cope less well with tasks that require patience and sustained attention. Glass (1977) describes a number of studies from which it becomes apparent that type A subjects in contrast to type B subjects, have a tendency to perceive time as passing more slowly when performing a time estimation tasks, have more difficulties in performing a delayed response task in which they have to wait at last twenty second before giving a response and perform more poorly on a reaction time task with long inter-trial intervals. So it seems plausible that type A subjects will cope less well with the conditions typical of a vigilance task.

During vigilance tasks, task performance and the level of physiological activation usually decline over time. We expect that type A subjects find it more difficulty to maintain their performance than type B subjects given the observations made by Glass (1977). Whether they also show a faster decline in level of physiological activation is less obvious. It is quite possible that type A subjects, given their high need of achievement, will try harder to counteract the decline in performance by putting more effort in the task. This way of coping will result in a relative high level of physiological activation in type $\mathrm{A}$ subjects in combination with a poor performance. Some experimental evidence for this hypothesis does exist. Lundberg and Forsman (1979) report heightened cortisol excretion in male type A subjects during a monotonous vigilance task.

On the basis of these considerations the following hypothesis was formulated: when working on a vigilance task type $A$ subjects show a smaller decline in physiological activation and a larger decline in performance than type $B$ subjects. It should be noted that this hypothesis differs slightly from the ones tested in the previous chapter: it is not a so much a difference in general reactivity but more a difference in the specific way in which the subjects behave during the task. 
The second hypothesis tested is similar to the reactivity hypothesis tested in the previous experiment: when working on a vigilance task type $A$ subjects show a larger increase, compared to a baseline, in feelings of distress and effort, and in homonal, physiological and platelet activation, than type $B$ subjects.

To create different levels of difficulty in remaining vigilant the subjects were presented with four different versions of an auditory vigilance task. The versions differed in the number of stimuli that were presented, the event rate, and in the proportion of signals, the signal/non-signal ratio. This was done to test the following two hypotheses dierived from the saliency hypothesis: during the low evert tasks the differences between type A subjects and type $B$ subjects will be more prominent than during high event tasks; during the low signal/non-signal ratio tasks the differences between type A subjects and type B subjects will be more prominent than during high signal/non-signal ratio tasks.

\section{2}

\section{DESCRIPTION OF THE EXPERIMENT}

In this experiment a number of changes were made in the methods in order to avoid some of the difficulties discussed in section 2.7 .

\subsubsection{Subjects}

The subjects were selected the same way as in the first experiment but a different sample of the population was used. Again a request to participate to research on cardiovascular disorders was made, this time to all male inhabitants of Maastricht born in 1943 and 1944. Those who participated were, at the time of the experiment, between $39-41$ years of age just like in the previous experiment. $A$ number of questionnaires were sent to 800 men, a random sample of the approximately $60 \%$ that expressed their willingness to participate. Subjects for the experiment were selected from a group of 650 men who returned the questionnaires. Selection was based on the scores on the Jenkins Activity Survey. In order to increase the contrast between the type $A$ and the type $B$ subjects even more than in the first experiment, those subjects with scores in the lowest quartile were classified as type A subjects, while those with scores in the highest quartile were classified as type B subjects. Complete data were available for twelve type $A$ and twelve type $B$ subjects. On the basis of their $M Q$ scores, these twenty-four subjects could be divided into twelve exhausted and twelve vital subjects, using the median MQ score of the total sample of 650 subjects as a cutoff point. Table 3.2.1 shows the resulting subgroups of subjects. It should be noted that the JAS scores given in this table are based on the scores obtained when the subjects had filled in the JAS for a second time, at the time they actually participated in the experiment. Furthermore, the scoring method had changed slightly compared to the one used for the first sample of subjects. Therefore, the two sets of scores are not comparable. None of the selected subjects had current or prior complaints of a cardiovascular nature or used any form of medication, according to their own account. By looking at the number of subjects in each subgroup one can see that, like in the first experiment, the two risk factors were not independent: the type A subjects were more often exhausted and the type B subjects more often vital. 


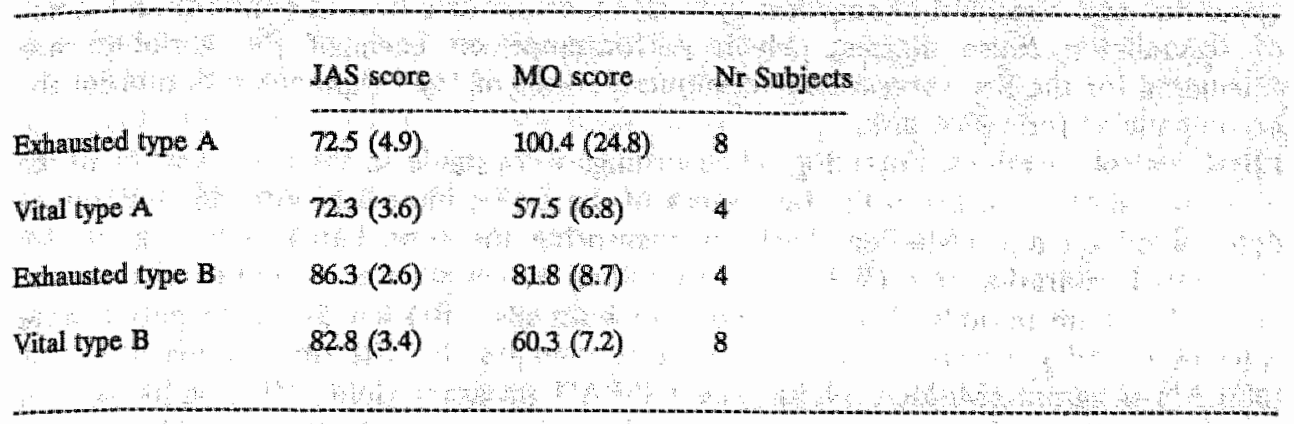

Table 3.2.1: Mean scores and standard deviations on the MQ and the from the subjects used in the vigilance experiments.

The Spearman correlation coefficient, which was used because the MQ scores were not normally distributed, between MQ scores and JAS scores was 0.29 . In contrast to the previous sample this correlation failed to reach significance although it pointed in the same direction.

\subsection{2 - Experimental conditions}

TV documentaries. Two documentaries of twenty five minutes each were shown.

Vigilance tasks. The task assigned to the subject was an auditory vigilance task. The subject had to attend to a series of clicks that had two levels of loudness. They had to respond when ever a click was weak Duration and pitch of each click were two mililiseconds and $1000 \mathrm{~Hz}$, respectively. Prior to each session, the sound level was set $40 \mathrm{~dB}$ above hearing threshold and the difference between signal and non-signal loudness was adjusted individually so that in a five-minute trial period, a performance of $90 \%$ correct detection was achieved by each subject.

Four versions of the task were used which differed in terms of event rate and signal rate. Event rate was either one click every two seconds or once in every six seconds. Signal rate was either one weak click in every five clicks or one click in every fifteen clicks on the average. The least difficult version was expected to be the high event rate high signal/non-signal ratio task in which relatively many stimuli were presented and where the subject had to react to a high proportion of the stimuli. The most difficult one was expected to be the low event rate low signal/non-signal ratio task in which stimulus presentation rate was low as was the proportion of those stimuli on which the subject had to react.

\section{2 .3}

\section{Dependent variables}

Subjective state variables. Subjective state was assessed with the same visual analog scales as in the previous experiment. From the raw values of seven scales, a distress and an effort score was obtained as in the previous experiment.

Performance variables. Performance variables were measured with a purpose built 
apparatus and consisted of reaction time (RT), errors of omission (misses) and errors of commission (false alarms). Mean performance on each of the variables was calculated for the five successive five-minute periods of each vigilance task and for the accompanying reference task.

Physiological variables. Physiological recordings were made in the same way as in the previous experiment. From the recordings of the $\mathrm{ECG}$, inter-beat-intervals (IBI) were determined on a beat-to-beat basis by measuring the time between two successive $R$-waves. Respiration rate ( $R R$ ) was determined by counting the number of respirations in 5-minute periods. Average inter beat intervals (IBI) per 5-minute period were calculated and a spectral analysis was performed on the IBI time series using the INSPAN program (Mulder, 1979). The INSPAN program yields HRV split up over specific frequency bands which are defined as "low-frequency-band" $(0.02-0.06 \mathrm{~Hz})$, "mid-frequency-band" (0.07-0.14 Hz) and "high-frequency-band" $(0.15-0.40 \mathrm{~Hz})$. Spectral power values for these bands were obtained for every 5-minute task and film period and for the 2.5 -minute resting periods.

Platelet activity. Venous blood samples were drawn with a syringe coupled to an indwelling catheter placed in the antecubal vein. The catheter was kept free from coaggulation by very slowly infusing isotonic fluid. In order to obtain a measure of in vivo platelet aggregation, a method described by Wu and Hoak (1974) and Deshmukh et al. (1976) was used. The blood sample was divided between two tubes containing ethylene-diamine-tetra-acid (EDTA) and two tubes containing EDTA and formalin. It was assumed that platelet aggregates would disintegrate in the first two tubes while they would remain intact in the second two. All four tubes were centrifuged for ten minutes at $180 \mathrm{~g}$, to obtain platelet-rich plasma. Then, after diluting the platelet-rich plasma 10000-fold, the number of particles in a sample of $0.50 \mathrm{ml}$ from each tube was determined, using a Coulter counter with a detection range of $7 \mu$ to $70 \mu$. With such a range only single platelets can be counted. Thus, an estimation of the total number of platelets could be obtained from the tubes containing EDTA alone, while the number of non-aggregated platelets could be estimated from the second set of tubes; the microaggregates were simply too large to be included in this count. To assess the amount of in vivo platelet aggregation, the aggregation ratio was calculated by dividing the number of freely circulating platelets by the total number of platelets counted in the tubes containing EDTA only:

Hormonal data. The blood samples from which levels of catecholamines and cortisol were to be determined were obtained in the same way as those from which platelet activity was assessed. After they were collected they were immediately transported to the department of pharmacology, where they were processed using a radio-enzymatic assay. Catecholamine levels were expressed as $\mathrm{pg} / \mathrm{ml}$ and cortisol levels as $\mu \mathrm{g} / \mathrm{m}$. Three values were obtained: one after the high event low signal/non-signal ratio task, one after the low event low signal/non-signal ratio task and one after that documentary, that was shown in the same part of the film session as the low signal/non-signal ratio tasks had been performed during the task session.

\subsubsection{Procedure}

In this experiment, three sessions were scheduled for each subject: one baseline 
session and two experimental sessions. During the baseline session, the two T.V. documentaries were shown. During the experimental sessions, the four versions of a vigilance task were used: two in each session. The order of the different sessions was balanced within the type A and type B group. Within each experimental session, event rate was kept constant and signal rate was balanced for order. For each subject the three sessions were helld a week apart and at the same time of day.

The experiment was carried out in a brightly illuminated soundproof room. The subject was seated in a reclining chair and was provided with earphones. The recording equipment and the experimenter were in an adjoining room.

The experimental sessions were divided into the following nine steps which are also shown in figure 3.2.1. After the subjects entered the laboratory, preparations for the physiological recordings were made and a physician inserted an indwelling catheter via venous puncture (1). When all recordings were satisfactory and the catheter was properly placed, the first blood sample for platelet aggregation was taken and the self reported state scales were filled in. Then the subjects were trained in the task until

(1) Preparation and baseline

(2) Training run vigilance task

(3) First resting period

(4) Vigilance I main task

(5) Vigilance I after task

(6) Second resting period

(7) Vigilance II main task

(8) Vigilance II after task

(9) Final resting period

Figure 3.21. Procedure of the task sesslons in the vigilance experiment.

performance criteria were met (2). This was followed by a resting period of 2.5 minutes (3). Next, the subjects were told to perform the vigilance task as well as possible by reacting as quickly as they could without making errors. They were told that the task would last for half an hour, while in fact it was over after twenty-five mimutes (4). Within twenty seconds after the task was over, a second blood sample was taken from the catheter. After this, the self reported state scales were presented for the second time and then the same task had to be performed for five more minutes (5). After completion of this reference task, there was another resting period of 2.5 minutes (6) and the second version of the vigilance task was assigned (7), again during a period of twenty-five minutes. When this task was completed a third blood 
sample was obtained and the self reported state scales were filled in for the third time. The second reference task followed (8) and the session was then completed by a final 2.5-minute resting period (9). The total duration of the session was approximately 100 minutes.

The control session consisted of six steps, as is shown in figure 3.2 After the arrival of the subjects, preparations were made for the physiological recordings and the catheter was placed (1). When all recordings were satisfactory, a blood sample was taken and the subjects filled in the self reported state scales. Then, a 2.5 -minute resting period was given (2), after which the first documentary was shown (3). When this film was finished, a second blood sample was taken, the subjects filled in the scales again and a second 25 -minute resting period followed (4).

(1) Preparation for recording

(2) Pre film resting period

(3) First film

(4) Between film resting period

(5) Second film

(6) Post film resting period

Figure 3.2: Procedure during the film session In the wigllance experiment.

The second documentary was then shown (5), again followed by the taking of a blood sample and the filling in of the scales. A final $2.5 \mathrm{~min}$ resting period (6) concluded this session. The total duration of the control session was approximately ninety minutes.

\section{3}

\section{RESULTS I: VIGILANCE DECREMENT}

In this section the changes during the tasks are analyzed to test the following two predictions, based on the first hypothesis: during the performance of the vigilance tasks type A subjects will show a smaller decline in physiological arousal, which will manifest itself in a smaller increase in inter-beat-interval, a smaller increase in heart rate variability and a smaller decrease in respiration rate, than type $B$ subjects, and: during the performance of the vigilance tasks type $A$ subjects will show a larger increase in the number of missed signals and in the reaction time than type $B$ subjects. Furthermore, the two predictions based on the saliency hypoihesis were tested: during the low event tasks the above mentioned differences between type $A$ subjects and type $B$ subjects will be more prominent than during high event tasks, and: during the low signal/non-signal ratio tasks the above mentioned differences between type $A$ subjects and type $B$ subjects will be more 
prominent than during high signal/non-signal ratio tasks.

Differences between vital and exhausted subjects in change during the tasks on the same variables were also explored.

Finally a check was made whether the tasks did indeed result in the expected change in vigilance and in physiological activation.

The predictions were tested by means of analyses of variance. The model included three repeated factors (task-blocks with five levels, event rate with two levels and signal/non-signal ratio with two levels) and two group factors, $A B$ typology and level of exhaustion. Data from 24 subjects were available for the analysis of respiration rate and performance data. Inter-beat-intervals and heart rate variability data could be obtained from 23 subjects.

\subsubsection{Main vigilance effects and the influence of task characteristics}

Initial reactions. To see how the subjects reacted to the beginning of the task $\mathrm{i}_{\mathrm{i}}$ the pre-task resting period and the first task period were compared. In table 3.3.1 the results are presented. At the start of the vigilance task all subjects reacted with an increase in respiration and a decrease in heart rate variability in all three frequency bands. No change in the inter-beat-intervals was found.

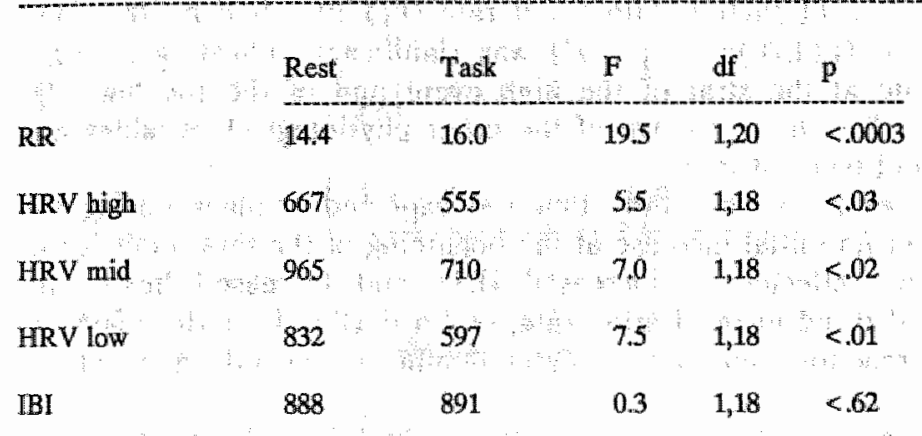

Table 3.3.1: Mean values of physiological variables during pre-task resting periods and the first 6minute block of the tasks (RR is resplration rate expressed in cycles per minute; HAV high is heart rate variablily with a frequency of $13-25$ Hertz expressed as \% variation*10; HRV mid is heart rate variability with a frequency of 8-12 Hertz expressed as \% variation*10* HAV low is heart rate varlabllity with a frequency of 1-7 Hertz expressed as of variation" 10" IBI is inter-beat-interval expressed in milliseconds).

General vigilance decrement. In order to check if the tasks produced the expected vigilance effects the main effects of task periods were tested. This resulted in changes in almost all physiological variables during the course of the task. Respiration rate was the only variable that did not change significantly but an interaction with event rate was found $(F(4,80)=2.5 p<.05)$ indicating that a decrease was found during the high event condition only. IBI clearly became longer during the task $(F(4,76)=12.5$ 
$p<.0001)$ and heart rate variability increased in all three frequency bands. The significance level of the increase in the low frequency band was $F(4,76)=4.3 p<009$, of that in the mediun frequency band $F(4,76)=3.4 \mathrm{p}<.03$ and of that in the high frequency band $F(4,76)=3.8 \mathrm{p}<, 02$. The performance vatiables also changed during the task. Reaction time increased $(F(4,80)=5.9 \mathrm{p}<.0009)$ and the number of missed signals increased too $(F(4,80)=14.8 p<00001)$. In figure 3.3 the changes during the tasks are shown separately for the type $A$ and $B$ subjects.

Efrect of tasks characteristics. To see whether the four versions of the vigilance task had a different effect on the vigilance decrement, the interaction between event rate and task periods and those between signal/non-signal ratio and task periods were tested. With the exception of respiration rate no consistent differences were found in the rate of change of the physiological variables when high and low event rate conditions were compared. During the low signal/non-signal ratio conditions only the increase in variability in the low frequency band was more pronounced $(F(4,76)=3.2$ $p<.05$ ). The absence of an influence of task characteristics made us wonder whether the decrease in physiological activation was not just a time-on-task effect. Therefore, the above mentioned analyses were repeated with the appropriate film periods as covariates. Exactly the same effects were found with and without covariates so we may conclude that the decrease in physiological activation was specific for the tasks.

The change in respiration rate at the beginning of the tasks was also affected by the task characteristics: Both the interaction with event rate $(F(1,20=10.8 \mathrm{p}<, 004)$ and with signal/non-signal ratio $(F(1,20)=6.5 \mathrm{p}<.02)$ was significant indicating a larger increase in respiration rate at the strat of the high event and of the the high sig. nal/non-signal ratio tasks. The initial change of the other phystological variables was not affected by the different types of task.

Conclusion. From these results we conclude that the expected changes during the tasks had taken place: after an initial increase at the beginning of the task a decline in physiological activation as reflected in increased IBI's and increased heart rate variability and a downward trend in respiration rate, and a decline in performance as reflected in an increased reaction time and a greater number of missed signals at the end of the task.

No consistent evidence of a generally stronger decline in physiological activation or a stronger performance decrement during the low signal/non-signal ratio tasks or the low event rate task was found. So the expected influence on vigilance decrement of the different task versions could not be established.

\section{3 .2}

Differences in vigilance decrement between type A and B subjects

To see whether differences in vigilance decrement, irrespective of the task characteristics, between type $A$ and $B$ subjects could be found, the interaction between AB typology and task periods were tested. To test the third and fourth prediction, the interaction between $\mathrm{AB}$ typology, task periods and either event rate or signal/nonsignal ratio was tested.

Overall differences in vigilance decrement. On respiration rate and inter beat interval no difference in rate of change was found. On heart rate variability however type $A$ 

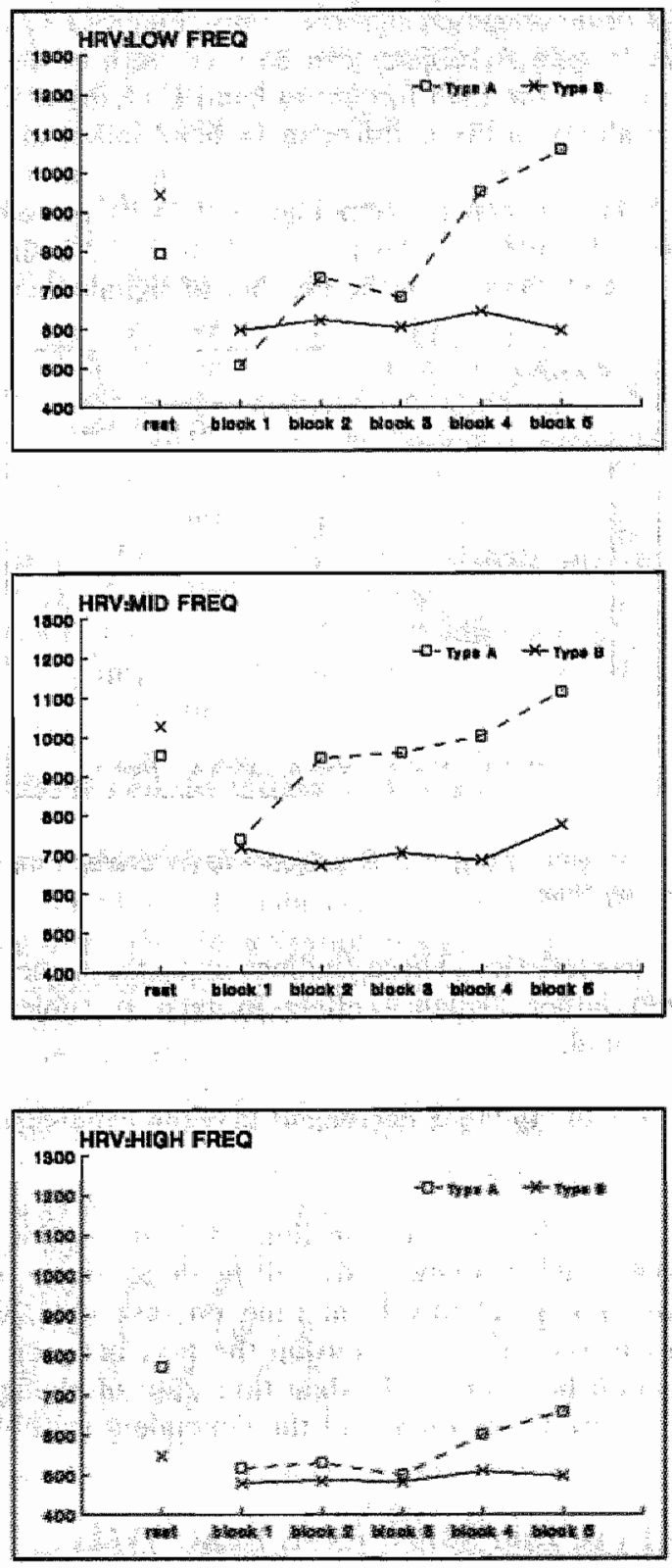

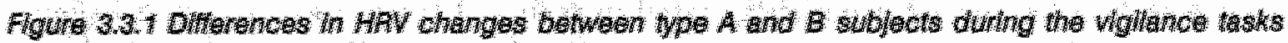

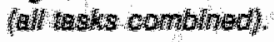


subjects had the most pronounced change over time. Figure 3.3.1 shows that the larger increase in variability in type $A$ subjects can be seen both in the low frequency band $(F(4,76)=4.1 \mathrm{p}<.01)$ and in the high frequency band $(F(4,76)=3.0 \mathrm{p}<.05)$ whereas the higher increase in yariability in the mid-frequency band failed to result in a significant interaction.

A significant interaction between $\mathrm{AB} /$ typology and tasks periods was found on the number of missed signals $(F(4,80)=2.6 p<05)$. Figure 3.3 .2 shows that the type $A$ subjects show a more rapid increase in the number of signals they miss.

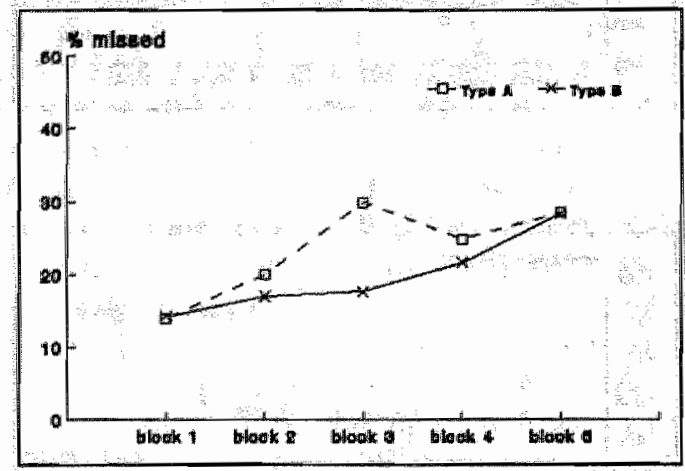

Figure 3.3.2: Difference between type $A$ and $B$ subjects in percentage missed signals during the Willance tasks (all tasks comblned).

Influence of tasks characteristics. These findings were the same for all four types of tasks so the expected larger vigilance effect in type A subjects during the more difficult task was not found.

\subsection{3}

\section{Differences in vigilance decrement between exhausted and vital subjects}

The same analyses as in the previous section were done this time with level of exhaustion as grouping variable. Only an overall tendency in the exhausted subject to react somewhat slower independently from time on task $(F(1,20)=2.7 p<.11)$ was found. No differences in rate of change during the task between exhausted and vital subjects or an interaction between vital exhaustion, rate of change and event rate or signal/non-signal ratio were found on any of the dependent variables.

\section{4}

\section{RESULTS II: DIFFERENCES IN REACTIVITY}

In this section the second hypothesis, stated in section 3.1: when working on a vigilance task type A subjects show a larger increase, compared to a baseline, in feelings of distress and effort, and in hormonal, physiological and platelet activation, than type $B$ subjects, is tested. Furthermore, the two predictions based on the saliency hypothesis i.e. during the low event rate task the differences between type $A$ and $B$ subjects will be more 
pronounced than during the high event task; and: during the low signal/non-signal ratio task the differences between type $A$ and $B$ subjects will be more pronounced than during the high signal/non-signal ratio task, are tested.

Possible differences between vital and exhausted subjects in the change in levels of catecholamines, cortisol and platelet from film to task are also explored.

Finally, we look at the main effects of condition to see whether the mean task and film values differ.

In order to be able to compare the hormonal and platelet aggregation variables with the physiological variables, mean values of the physiological variables were calculated using the values obtained during the last 5-minutes block of the tasks and the films.

Complete data from 24 subjects were available. Analyses of covariance was performed on each of the dependent variables, except the hormonal data, using a $2 \times 2 \times 2 \times 2$ design. $\mathrm{AB}$ typology and level of exhaustion were included as group factors, and the two task characteristics, event rate and signal/non-signal ratio, as repeated factor. For the hormonal data a $2 \times 2 \times 2$ design was used, omitting signal/non-signal ratio as a factor. The mean value of the two film blocks was used as covariate.

The first hypothesis was tested by looking at the main effect of AB typology. The two predictions were tested by inspecting the interaction between $A B$ typology and either event rate or signal/non-signal ratio.

\subsubsection{Main task effects and the influence of task characteristics}

First of all we wanted to test whether the tasks actually differed from the films in the amount of activation found in all subjects, and whether the task characteristics affected the size of the difference in activation between task and film.

We tried to answer the first question by looking at the main effect of task vs film. The answer to the second question was obtained by looking at the main effects of event rate and signal to non-signal ratio.

Subjective state variables. In order to test whether the tasks and the films were experienced differently the state values reported after each task and film were analyzed in the same way as the physiological variables. In table 3.4.1 the results are shown. For distress, a higher score, compared to the film, was found for all tasks but signal to non-signal ratio had a significant effect $(F(1,20)=6.9 \quad p<.02)$. A more pronounced difference during the high signal to non-signal ratio tasks. The difference in effort was not very large. Only during the high event rate low signal/non-signal ratio task the separate comparison between task and film was significant but the effort scores were higher during all four tasks;

Physiological variables. Some effects of event rate and signal/non-signal ratio were found indicating that during some tasks differences in the physiological variables might be found. For respiration rate an effeet of both event rate $(F(1,20)=8.9 p<007)$ and of signal to non-signal ratio $(F(1,20)=4.4 \quad p<.05)$ was found. For heart rate variability an effect of signal to non-signal ratio was marginally significant in the low frequency band $(F(1,19)=3.9 \mathrm{p}<.06)$. In the other two frequency bands no significant ratio effect was found. However in all three band more suppression was found during the high signal to non-signal ratio. 


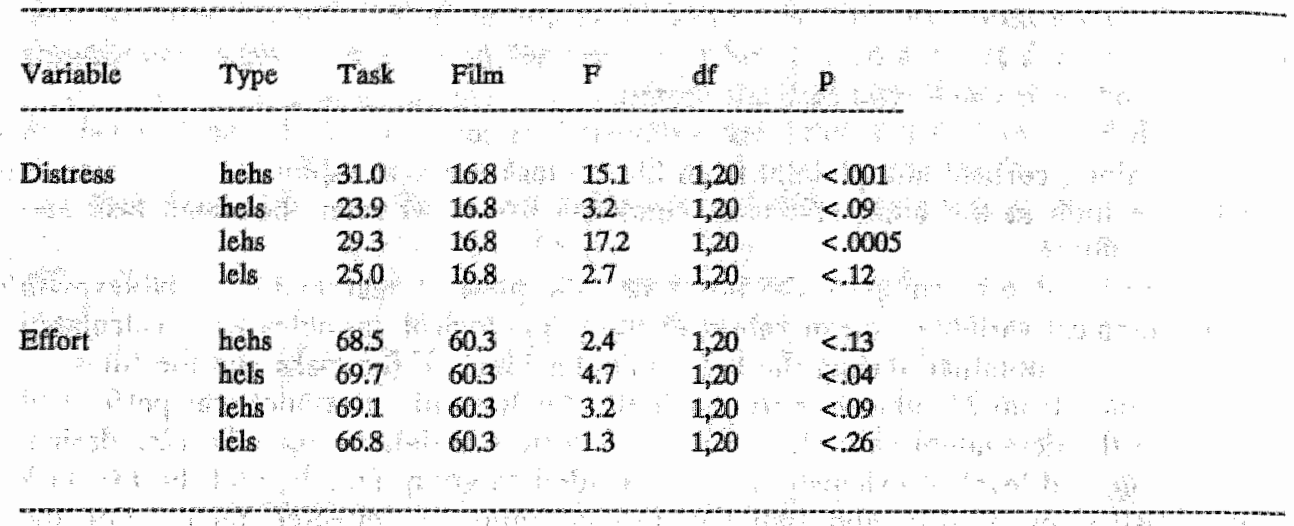

Table 3.4.1: Subjective state scores of the tour tasks and the mean of the two films from all subjects. (hohs is high event rate high signal to non-signal ratio, hels is high event rate low signal to nonslgnal ratio; lahs is low ovent rate high signal to non-signal ratlo; lols is low ovent rate low signal to non-signail ratto).

In table 3.4.2 the values obtained during the last 5-minutes blocks of each of the tasks and the of the film are shown. From this table it can be seen that only during the last block of the high event rate high signal/non-signal ratio task there is some indication of a higher physiological arousal compared to the film. In this condition the most pronounced difference between task and film is found on the physiological variables.

Hormones. For none of the three hormones a difference between the two tasks and the film session was found nor was there a main effect of event rate.

Platelet aggregation. No difference in platelet ratio between the four tasks and film was found. Nor was there an effect of event rate or signal/non-signal ratio.

\subsubsection{Differences in reactivity between type A and $B$ subjects}

Subjective state variables. No main effect of $\mathrm{AB}$ typology was found for distress or effort.

Physiological variables. Only in the low frequency band of the HRY an effect of $A B$ typology was found $(F(1,18)=4.1 \mathrm{p}<.06)$ indicating that, during all the tasks, type $A$ subjects had less suppression of HRV than type B subjects. The means of the four tasks, corrected for the mean film value, was 988 for type A subjects and 590 for type $B$ subjects (values are expressed as $\%$ variation ${ }^{*} 10^{2}$ ).

Hormones. No significant effect of $A B$ typology was found on any of the three hormones. There was however a tendency in type A subjects to have a higher noradrenaline concentration when the values of all three sessions were combined. When type A and B subjects were compared irrespective of their level of exhaustion, a marginally significant main effect was found $(F(1,22)=3.91 \quad \mathrm{p}<.06)$. When both group factors were used simultaneous in the comparison, thereby correcting for the fact that type A subjects were predominantly exhausted, 


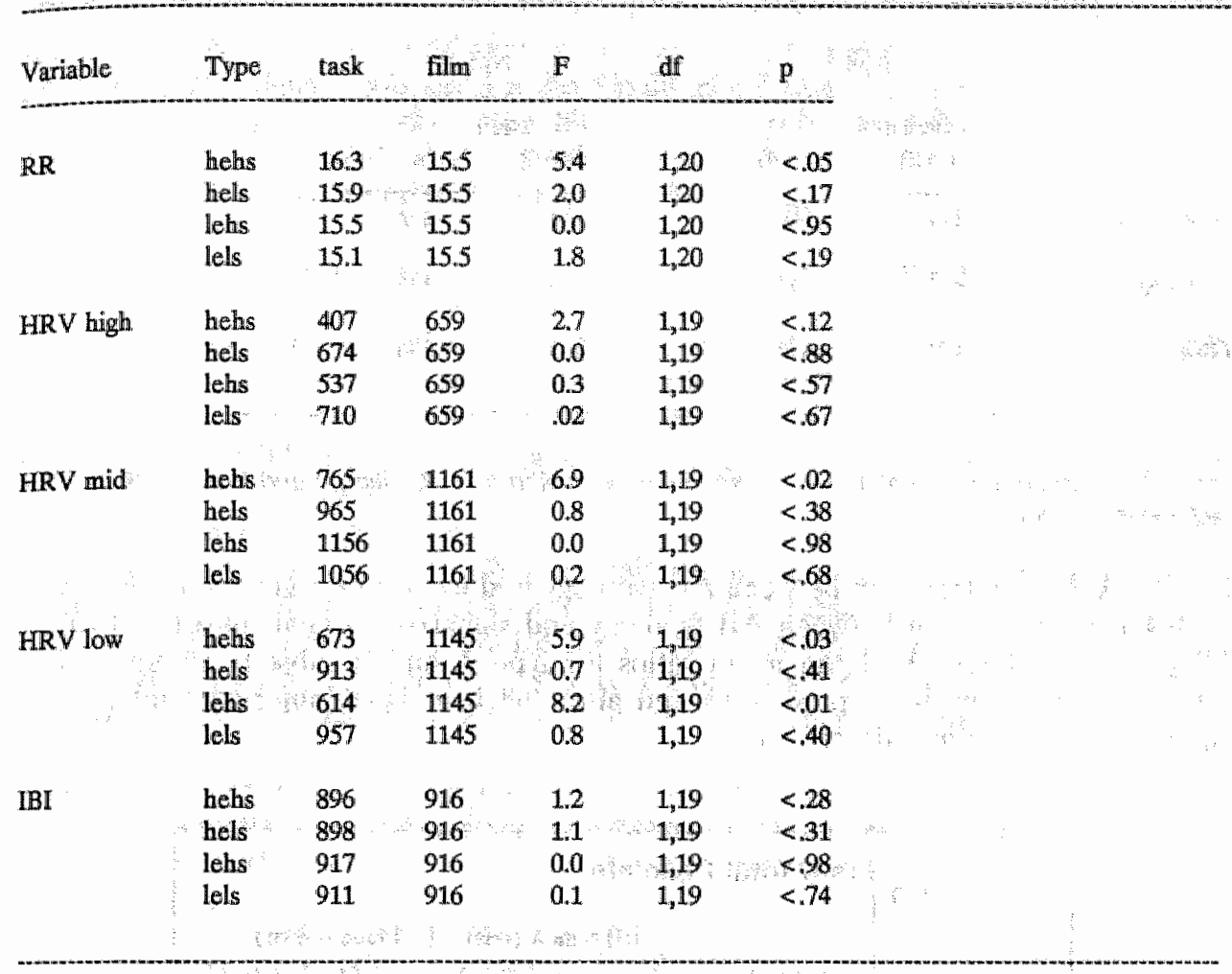

Table 3.4.2: Mean values of physiological variables for all subjects based on the values obtained during the last 5-rinitites blocks of the tasks and the film, (RR is resplration rate expressed in cycles per minute; HRV high is heart rete variablity with a frequency of 13-25 Hertz expressed as \% variation*10; HRV mid is heart rate variability with a frequency of $8-12$ Hertz expressed as \% varlation*10; HRV low is heart rate varlabllity with a requency of $1-7$ Hertz expressed as \% variation*10; IBI is inter-beat-interval expressed in millseconds; hehs is high ovent rate high signal to non-signal ratio; hels is high ovent rate low signal to non-signal ratlo; lehs is low ovent rate high signal to non-signal ratio, (els is low event rate low signal to non-slgnal vallo).

this effect was no longer significant $(F(1,20)=2.28 p<.15)$. From table 3.4 .3 it can be seen that it are mainly the exhausted type A subjects that are responsible for the overall higher noradrenaline values in type A subjects. When the eight exhausted type A subjects were compared to the remaining sixteen subjects, consisting of vital type $\mathrm{A}$ and exhausted and vital type B subjects, a clear difference was obtained $(\mathbf{F}(1,22)=8.21$ $\mathrm{p}<.01)$. The mean noradrenaline concentration of the exhausted type A subjects was $451 \mathrm{pg} / \mathrm{l}$ while that of the other subjects was $333 \mathrm{pg} / \mathrm{l}$, a difference of approximately one standard deviation.

Platelet aggregation. The main effect of $\mathrm{AB}$ typology was not significant so in generall type $A$ subjects did not show a more pronounced difference between the ratio scores obtained after the task and after the film condition than type B subjects. 
type A

type $\mathbf{B}$

\begin{tabular}{lcccc}
\hline $\begin{array}{l}\text { exthausted } \\
(\mathrm{n}=8)\end{array}$ & $\begin{array}{c}\text { vital } \\
\mathrm{n}=4)\end{array}$ & $\begin{array}{l}\text { exthausted } \\
(\mathrm{n}=4)\end{array}$ & $\begin{array}{c}\text { vital } \\
(\mathrm{n}=8)\end{array}$ \\
\hline 453 & 307 & 374 & 326 \\
421 & 373 & 306 & 335 \\
479 & 339 & 303 & 336
\end{tabular}

Table 3.4.3: Mean noradremaline concentration of exhausted and wital type $A$ and $B$ subjects after the tasks and the film.

Neither was the interaction between $A B$ typology and event rate significant. There was however an interaction between $A B$ typology and signal/non-signal ratio $(F(1,20)=$ $5.2 \mathrm{p}<.03$ ). In figure 3.4.1 the mean ratios for type $\mathrm{A}$ and $\mathrm{B}$ subjects obtained after the high signal/non-signal ratio tasks and after the low signal/non-signal ratio tasks, corrected for the films, are shown.

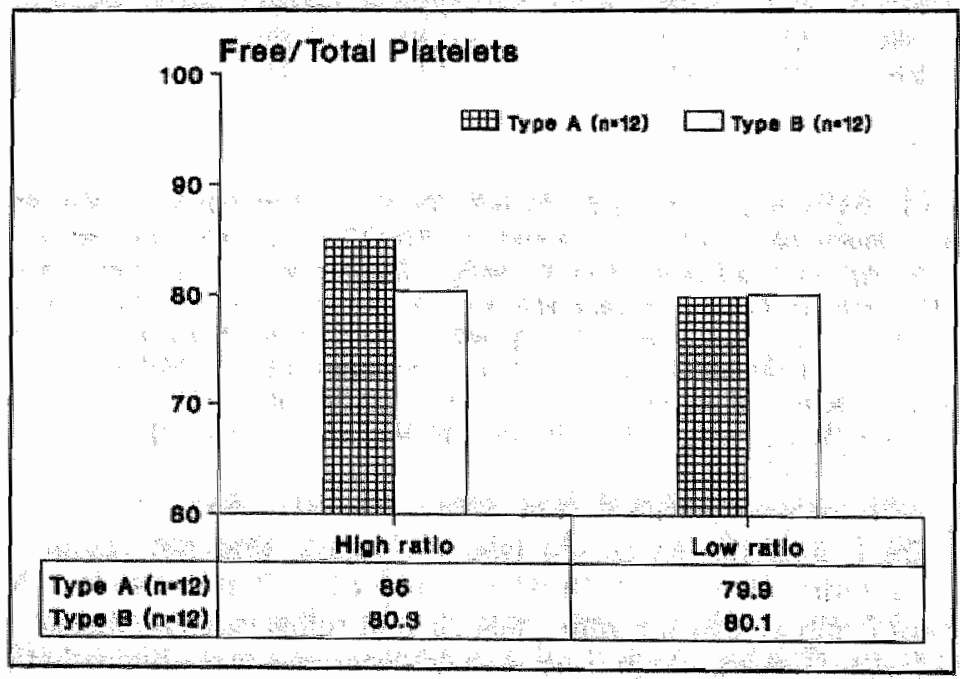

Flgure 3.4.1: Ratlos of freely cliculating plate/ets and total platelets from type $A$ and $B$ subjects obtahned after the high and the low signal/hon-signal ratio tasks. Nalues are corrected for film vallues.)

Under the high signal/non-signal ratio condition type A subjects tended to have a higher platelet ratio, meaning less aggregation, than type $B$ subjects. Under the low 
signal/non-signal ratio condition type $A$ and $B$ subjects hardly differed. When type $A$ and $B$ subjects were compared separately after each of the conditions only after the high signal/non-signal ratio tasks a marginally significant difference in platelet ratio was found $(F(1,20)=4.0 \mathrm{p}<.06)$ the direction of which was against expectation. So even although an interaction, including signal/non-signal ratio was found, after decomposition of this interaction no evidence for the second prediction was found.

\section{4 .3}

Differences in reactivity between exhausted and vital subjects

Subjective state variables. No main effect of level of exhaustion was found. There was, however, a tendency in the exhausted subjects to have a higher distress score during all the three sessions than the vital subjects: 28.3 vs $15.9(\mathrm{~F}(1,20)=3.4 \mathrm{p}<.08)$. Physiological variables. Again no significant main effects or interactions were found that included level of exhaustion.

Hormones. No significant main effect of level of exhaustion was found for any of the three hormones. There was however a tendency in the exhausted subjects to have a higher concentration of noradrenaline in all the three sessions. This was again mainly caused by the relative high noradrenaline concentration in the exhausted type $A$ subjects as has been shown in the previous section. When exhausted and vital subjects were compared irrespective of $\mathrm{AB}$ typology the difference was marginally significant $(F(1,22)=3.1 \quad \mathrm{p}<.09)$ but when the comparison was made with a correction for $A B$ typology it was no longer significant $(F(1,20)=1.53 \mathrm{p}<.23)$.

Platelet aggregation. No main effect or interaction that included level of exhaustion was found to be significant.

MAIN CONCLUSIONS AND DISCUSSION

From this experiment the following conclusions are drawn:

1 The vigilance tasks produce the expected changes: an initial rise in actiwation is followed by a decline of activation and performance during the course of the task.

2 Some differences in vigilance effects occur between type A and B subjects: A larger decline in physiological aetivation and an earlier decline in performance is found in type $\mathrm{A}$ subjects, indicating that they are less able to maintain their vigilance than type B subjects.

3 The manipulation of the tasks characteristics did not affect the vigiliance effects in general.

4 No clear difference between the values at the end of the task and those at the end of the baseline of the biological variables is found.

5 No clear difference between exhausted and vital subjects is found when they work on vigilance tasks. 
6 Exhausted type A subjects have a higher level of plasma noradrenaline regardless the experimental condition.

7 The changes in HRV due to the tasks are independent of IBI.

Differences in vigilance effects. How well do the observed differences between type A and $B$ subjects support the prediction that type $A$ subjects will show a smaller decine in physiological activiation and a larger decline in performance than type B subjects. Regarding the physiological changes, if anything, evidence for the opposite was found. The type B subjects showed a more or less constant suppression of heart rate variability in contrast to the type A subjects who showed a similar suppression at the start of the session but their variability clearly increased during the task, particularly in the low and in the high frequency band.

This finding was specific for the task sessions since during the film session no difference between type A and B subjects was found in the way their HRV changed during the task. In contrast to what happened during the tasks, there was no suppression at the beginning of the film and no significant interaction between session periods and $A B$ typology which would indicate a difference in rate of change between type $A$ and $B$ subjects while watching the films.

No difference between type A and B subjects was found in the rate of change of the IBI. Both groups showed a similar lengthening of IBI churing the tasks. In this respect IBI differed from HRV since on the latter variable the two groups did differ. Beside the fact that these two measures did not show similar differences between type $A$ and $B$ subjects in the way these two groups changed during the task, differences between IBI and HRV were also found at the start of the tasks, both at the beginning of the task sessions and after the break. Here, in both cases, HRV was suppressed in all three frequency bands but IBI was not affected by the change in condition.

The finding that changes in HRV are independent from changes in heart rate adds to the puzzle of explaining the underlying mechanisms of HRV in terms of changes in autonomic balance. Clearly the knowledge of the regulation of heart rate does suffice to be able to understand the factors which influence HRV. The observed suppression of HRV at the start of the vigilance tasks is in line with the empirical relation that is usually found between increase in mental load and suppression of HRV (Mulder and Mulder, 1981).

When looking at the performance data; type A subjects do show a larger increase in the number of missed signals in the first part of the task but in the second part of the task the percentage of missed signals is equal for both groups. When we compare the change in reaction time no clear differences between type $A$ and $B$ subjects were found.

The different vigilance effects found in type A subjects were independent of the type of task. However, given that only a weak effect of signal to non-signal ratio on vigilance decrement was found, the manipulation of tasks characteristics was probably not strong enough to influence the difference between type $A$ and $B$ subjects,

A tentative post hoc explanation of the differences found between type A and B subjects during the vigilance tasks is that type A subjects are less able to stay alert and stop investing effort towards the end of the tasks. This leads to a poorer performance 
and to an increase in HRV. Type B subjects at the other hand keep on trying to remain vigilant, which results in a continuous suppression of HRV.

This explanation would be more convincing if the blood platelet and the hormonal data had provided corroborating evidence. However these variables were not even sensitive enough to differentiate between task and film sessions.

No evidence was found for a difference in vigilance decline between exhausted and vital subjects.

Differences in reactivity. As far as the difference in reactivity between type A and B subjects are concerned no evidence was found for the three predictions of higher activation levels in type $A$ subject tested in this experiment. The difference in reactivity between type A and B subjects on the low frequency band of the heart rate variability even was against expectation.

No differences at all could be obtained when exhausted and vital subjects were compared.

When the initial reactions during the tasks were examined a similar lack of difference in response was found when either type A and B subjects or vital and exhausted subjects were compared.

Lack of differences between task and film values of hormones and platelet aggregation. Even though in this experiment the assessment of the hormonal values was more closely related to the tasks and the film than in the first experiment, no overall difference between the experimental conditions was found for these variables.

The absence of a difference in plasma hormone values after the vigilance task seems to be at variance with the results from Lundberg and Forsman (1979) who found higher excretion rates of adrenaline and cortisol in all subjects and also higher cortisol excretion in type A subjects than in type B subjects.

However, our experiment differed in two aspects from theirs. First the degree of monotony differred. Although stimulus rate was roughly comparable, the signal/nonsignal ratio they used was two respectively six times lower than the one we used. Furthermore, the duration of their task was more than twice as long as ours. The adrenal-cortex tends to become involved mainly when stress is more intense and/or of longer duration (Seley, 1976) and this might explain our negative findings with regard to cortisol. Second the method of hormone assessment differed. They used urinary hormone samples and we used plasma samples taken at the end of the tasks.

Regarding the difference in sensitivity of plasma vs urinary cortisol virtually no data are available. In a study of Bohlin e.a. (1986) plasma cortisol was unaffected by mental arithmetic under time pressure but this ties in with the insensitivity of urinary cortisol to moderately intense laboratory tasks.

A difference between urinary catecholamines and plasma catecholamines is that the latter only give information over a short period, 1-3 minutes preceding the sampling, while the former are related to the mean activity of the total sample period which is usually more than one hour. From a number of studies (Forsman and Lindblad, 1983; Ward et al., 1983) it became clear that these plasma catecholamine values follow the adaptation to stress closely, and that adaptation to moderately intense stress does occur.

The same lack of difference between task and film was found for the index of platelet aggregation in vivo. Apparently this variable is less sensitive to changes in experimen- 
tal conditions than the self reported state variables which did differentiate more clearly between the film and the task sessions.

In this experiment measurements of the platelet aggregation index were taken at the beginning of each of the three sessions which allowed for a reliability test on this index. Alpha coefficients of the two constituents of the ratio were both satisfactory: Total number of platelets had an alpha of .87 and the number of freely circulating platelets had an alpha of 80 . However the reliability of the ratio itself proved to be highly unsatisfactory: the alpha was $-0,10$.

Since a reasonably high reliability is necessary to be able to show differences between groups or conditions, a more reliable index of platelet aggregation in vivo has to be used for a test of the hypotheses stated in this section.

We decided to use the number of freely circulating platelets as dependent variables in a post hoc test of the same hypotheses. Essentially the same results were obtained: No difference between the tasks and the film and no evidence for any of the bypotheses. Even this more reliable index of platelet activity is not sensitive enough to differentiate between the conditions used in this experiment.

Given that on the physiological variables a clear difference in level of activation could only be established at the end of the task that required that most decisions and reactions, the possibility that due to the monotony of the tasks the decline in activation was such that levels similar to the baseline condition were present during most of the task should be considered seriously. This might have played a role in the lack of difference on the hormonal and platelet variables between the task and the film session.

Other findings, An interesting finding in this experiment was the relative high noradrenaline concentration of the exhausted type A subjects.

This finding is important for two reasons. Firstly it shows that, at least in this experiment, the combination of the two risk factors leads to a generally higher level of noradrenaline. Secondly it shows that when subjects are compared using either $A B$ typology or level of exhaustion without correction for the other risk factor, erroneous conclusions can be made.

In this experiment neither $A B$ typology nor level of exhaustion affected noradrenaline levels when a correction was made for the influence of the other risk factor, but both did when the proper correction was not made. 
In this chapter a summary of the evidence found to support the main hypotheses tested in the two experiments, performed for this thesis, is presented. Our findings will be related to the findings reported in a number of reviews (Matthews, 1982; Dembroski et al., 1983; Holmes, 1983; Houston, 1983; Krantz and Manuck, 1984; Wright et al, 1985; Contrada and Krantz, 1988; Harbin, 1989). A critique of both the methods used by the reviewers and the reviewed articles themselves is given. Finally, some measures for dealing with the methodological problems encountered will be discussed.

REVIEW OF THE EVIDENCE SUPPORTING FOUND TO THE MAIN HYPOTHESES AND PREDICTIONS

The two main questions that were dealt with in our experiments were (1) whether, in general, type A subjects would show a larger increase in subjective and in physiological activation scores, reflecting SAM activity, than type B subjects when exposed to a stressor and (2) whether hyperreactivity in type A subjects would be seen more clearly in tasks with a high saliency.

In the first experiment no support was found for the hypothesis of a larger SAM reactivity in type A subjects in general. When the mean level of activation of type A and type B subjects during the task session was compared to that during the film session, both groups reacted in the same way.

Limited evidence was obtained for the saliency hypothesis. During and after feedback on the performance of the subjects, some differences in reactivity emerged. After they were criticized type A subjects reacted with a stronger increase in subjective arousal and physiological activation than type B subjects. The experimental manipulation of time pressure did not influence differences in reactivity between type $A$ and type $B$ subjects. It should be noted that the manipulation of time pressure did not affect physiological activation in general; thus, the lack of a clearer difference in reactivity during medium levels of time pressure might be explained by the limited effects of the experimental manipulation. The effect of passive coping conditions was not very strong. Only the combination of criticism and forced passivity led to a clear difference in reactivity.

In the second experiment again no support was found for the first hypothesis. Neither when task and film level were compared nor when the pre-task rests and the first block of the tasks were compared a larger increase in activation was found in type A subjects. However, Type A subjects did react differently during the monotonous vigilance tasks, not with the expected higher level of physiological activation but with a faster decrease in activation and performance during the tasks. This finding corroborates to some extend the saliency hypothesis. Yet, the experimental manipulations of the degree of monotony 
did not affect the degree of difference between type A and type B subjects. However, it is doubtful whether the manipulation of the tasks characteristics did actually result in a difference in degree of vigilance decrement in general, since differences in signal ratio affected only one of the dependent variables and manipulation of event rate had no influence at all. This precludes an unequivocal interpretation of the absence of the effects of task characteristics on the difference in vigilance behavior between type $A$ and $B$ subjects.

In addition to testing the predictions derived from the two main hypotheses, differences between type A and B subjects in PAC reactivity and differences between exhausted and vital subjects in both the PAC and the SAM systems were explored.

In none of the experiments differences between type $A$ and $B$ subjects in PAC reactivity were found.

During the first experiment, some differences between vital and exhausted subjects emerged that were tentatively interpreted as a lesser ability of exhausted subjects to cope with tasks that were highly demanding. More specifically they became less accurate when time pressure was increased. The same decrease in accuracy was found after they had been criticized. The finding of faster deterioration with increasing time pressure in exhausted subjects was replicated in a small study comparing eight vital and eight exhausted subjects which were selected on the basis of upper and lower quartile scores of the Maastricht Questionnaire (Portegies, 1983).

Furthermore, an unexpected higher level of 17-OHCS and platelet activation was found in the exhausted subjects during the film session. This finding could not, however, be replicated, neither in the above mentioned small study nor in the second experiment, even though the conditions were virtually the same.

Finally we looked at the interaction between type $\mathrm{A}$ behavior and vital exhaustion. Twice the exhausted type A subjects showed a biological activation pattern that was not found in the other three subgroups.

In the first experiment the platelets of exhausted type A subjects were more easily triggered after the film session. When, in the second experiment, we tested the hypothesis of increased in vitro activation in exhausted type A subjects during stress, no evidence could be found to support it. However, the low reliability of the index used for platelet activity precludes a clear answer to this question.

The second difference was the higher level of noradrenaline in the exhausted type A subjects found during the second experiment. Some precaution should be taken in generalizing this finding since no corroborating evidence for a higher levell of activation during this experiment was found in these subjects.

Looking at the results of the two experiments, no evidence for a higher reactivity in type A subjects in general was found. Some evidence was obtained for the saliency hypothesis, and a number of interesting, but not very easily explainable, findings with regard to vital exhaustion emerged out of the various analyses. 
How should one judge these results in comparison to those of other studies? In order to answer this question, we will look at some reviews of experiments regarding reactivity and type A behavior. Although hardly any studies have been published regarding stress patterns in exhausted individuals, the concept has been included in two dissertations. The findings of these studies will serve as a frame of reference for our results.

\section{4,2.1 Type A behavior}

Since 1980 quite a number of reviews concerning the reactivity hypothesis have been published. In a recent one, Harbin (1989) describes the diversity of the conclusions reached by the reviewers regarding the presence of greater reactivity in type A subjects: "inconclusive evidence" (Dembroski et al, 1983), "larger reactivity in type A subjects if one uses the appropriate variables and the structured interview to assess $\mathrm{A} / \mathrm{B}$ typology" (Matthews, 1982; Houston, 1983), and "no clinically important differences" (Holmes, 1983). Wright et al (1985) reach a conclusion similar to that of Matthews, but they also stress the importance of choosing the appropriate stimulus conditions in order to elicit larger responses in type A subjects.

Harbin (1989) regards the imperfection of the narrative review method as the cause of this diversity. He prefers a quantitative meta-analysis and concludes on the basis of such an analysis that the classification instrument, the task conditions, and the choice of the dependent variables all influence the degree of difference in reactivity between type $A$ and B subjects.

Thus, in spite of some diversity in the conclusions about the strength of the evidence for greater reactivity in type A subjects, the reviewers do agree to a large extent that the original hypothesis of generally increased reactivity in type A subjects should be replaced by more precise hypotheses. The stimulus conditions under which the two groups are expected to differ and the dependent variables that are expected to change should be taken into account Moreover, attention should be given to the choice of the classification instrument. Finally, the search for specific subgroups that are especially reactive should be continued.

Both the conclusions based on an analysis of the older literature, presented in the introduction of this thesis, and the results of our experiments agree with the conclusions of the recent reviews.

Regarding the appropriateness of the experimental condition, the larger reactivity in type A subjects after experiencing failure confirms the importance of testing the higher sensitivity to failure in type A subjects as suggested by Houston (1983). The higher vulnerability of type A subjects to vigilance effects also is understandable when one recalls the finding of Frankenhaeuser et al. (1980a) that type A subjects were less able to cope with the inactivity they experienced during the control session of an experiment. Furthermore, the observation in the second experiment that only the combination of being exhausted and type A led to a higher level of noradrenaline is an example of the positive effect of selecting a subgroup within the general type A population. 
Both in the dissertation of van Doornen (1988) and that of Vingerhoets (1985) the Maastricht Questionnaire was included. They used the same version as was used in our studies:

In van Doomen's study wital exhaustion was used as an independent variable. During stress a significant correlation was found with noradrenaline $(\mathrm{r}=32 ; \mathrm{n}=33 ; \mathrm{p}<.05)$ but not with adrenaline or heart rate. During a baseline session, no significant correlation was found. Higher levels of noradrenaline were also found in exhausted subjects in our second experiment, but when the influence of type A behavior was removed by using a stratified analysis, the relationship no longer remained significant In van Doornen's study, type A behavior and level of exhaustion were correlated (37), like in our studies. Because he used an analysis in which the effect of AB typology was not excluded, the possibility that, in his data, the association between exhaustion and noradrenaline can also be explained by the fact that high levels existed mainly in exhausted type A subjects, cannot be excluded.

In Vingerhoets' study, about the effects of stressful films, the Maastricht Questionnaire was used as a dependent variable and therefore, data on the relation between vital exhaustion and reactivity were not presented in his dissertation. However, he did explore the influence of vital exhaustion on changes in both physiological and neuro-endocrine variables during stress. These variables consisted of blood levels of adrenaline, noradrenaline, ACTH, cortisol, growth hormone and testosterone. Also include were a number of cardiovascular variables like heart rate, pulse transit time, T-wave amplitude, and an index of heart rate variability. Two additional variables were skin conductance level and fingertip temperature. On none of these variables a significant association with vital exhaustion was found (personal communication).

The available results regarding differences in biological reactivity between vital and exhausted individuals do not contrast with the, mainly negative, results we have found.

Why, then, don't we close this discussion, forget about vital exhaustion as an independent predictor of higher biological activity, and return to the laboratory to continue the search for other conditions that are relevant for type $A$ subjects and thus might give us significantly larger reactivity scores in type A subjects or; to be more precise, in exhausted type A subjects?

In order to answer this question we will play the role of devil's advocate and focus on the weak points of the arguments and methods used by both the researchers and the reviewers in their defense of a more elaborate version of the reactivity hypothesis, in order to test the robustness of their ideas.

The authors of the review articles tried to bring some order into the chaos of experimen- 
tal results. They argued that if one takes into account a number of important issues like the choice of classification instrument, the existence of a subset of type A individuals that are more reactive, the type of task used as a stressor and the choice of the dependent variables, more often significant results will be found. Most of the reviewers ullustrated their arguments by discussing the superiority of the SI method as compared with JAS in obtaining significant results or by giving a description of the task characteristics or the subgroup characteristics of the successful experiments. Furthermore, the fact that systolic blood pressure was more often found to differentiate between type A and B subjects than, for instance, diastolic pressure led them to suggest that it was the SAM system, and more specifically the beta-sympathetic system, that was involved in the hyperreactivity in type A subjects.

There are, however, grave shortcomings to this so-called "narrative" way of doing a review. Light and Pillemer (1984) list three main objections: the narrative review is subjective, it is scientifically unsound, because it often only takes "votes counts" when conflicting results have to be combined, and finally it is an inefficient way to extract useful information, particularly when a large number of studies is analyzed.

These objections are of particularly importance for the "narrative" reviews of the reactivity literature. In most of the reviews no formal tests of the conclusions regarding the influence of the classification instrument, the choice of experimental eonditions, and the appropriateness of certain dependent variables are provided, and the possible confounding effects of, for instance, the classification instrument on task characteristics have not been investigated.

To illustrate these points we will show that the task characteristics in successful experiments do vary considerably, that the assertion that it is predominantly the SAM system that is involved in the hyperreactivity is not very well founded, and that the selection of hyperreactive subgroups is not easy to accomplish.

Task characteristics. The tasks of "moderate levels of difficulty and/or competitiveness" that were claimed to lead most often to significant differences between type A and $B$ subjects tasks in fact vary widely. In one experiment a task in which $50 \%$ of the problems could be solved was labeled "moderately difficult" (van Schijndel et al., 1984) whereas in an other experiment the same label was given to a task in which $35 \%$ of the problems could be solved (Holmes et al., 1984), a task which was labeled as "easy" in the first experiment. "Moderately competitive tasks" that led to significant differences between type $A$ and $B$ subjects included reaction time tasks during which money could be lost (Perkins, 1984), a dual choice reaction time task - after criticism only - (Lulofs et al, 1986) and a subtest from the W.A.I.S (Holmes et al., 1982). This wide choice in tasks makes it rather difficult to determine the degree of difficulty or competitiveness of a particullar task.

Arguments for beta-sympathetic activation. Increased beta-sympathetic activation has been deduced from larger increases in heart rate and/or systolic blood pressure and/or catecholamines, variables that have been used most often. Evidence to support the statement that the larger reactivity should be understood mainly as a larger increase in beta- sympathetic activation has been based on each of these variables at some time but seldom on a combination of them in one and the same experiment.

The validity of this statement has also become questionable by some other experimental 
findings. A number of authors have reported differences in vasomotor reactions that were explained as allpha sympathetic over-reactivity (van Egeren, 1979; van Doornen, 1988). Others have found differences in heart rate variability, a measure that is generally interpreted as an index of vagal activity (Jennings and Choi, 1981; this thesis chap 3).

Selection of subtypes. The evidence that a clearer difference in reactivity can be found using subtypes is rather weak. Very often post hoc analyses, using subtypes, were performed when a comparison based on general type A scores did not prove successful. Two personality characteristics - hostility and speed /impatience - have been proposed as relevant subtypes within the general type $A$ concept. Drawing conclusions from the hostility literature is hampered by the numerous ways in which this concept is measured. They range from observation techniques using the A/B interview (Dembroski et al., 1978) to various scales of which the Cook and Medley scale (Cook and Medley, 1954) is the most widely used. Both positive (Weidner ef al, 1989) and negative results (Glass et al., 1983) have been found when testing for larger reactivity in hostile subjects.

Speed/impatience is a concept measured by a subscale of the JAS (Jenkins et al,, 1979), This subscale has recently been divided into two additional subscalles: irritability and impatience, on the one hand, and hard-drivingness and competitiveness, on the other. Only subjects seoring high on irritability and impatience were found to have somewhat higher reactivity scores (Öhman et al., 1989).

Conclusion. It can thus be concluded that differences in reactivity can be found while performing any of a number of tasks. These differences can be expressed in any of a number of variables, and usually one kind of subtype is more reactive than another. Unfortunately, however, these findings are mainly based on post hoc analyses and the consistency of the findings, both across and within experiments, is highly unsatisfactory.

If the systematic influences of the tasks and classification instrument on the estimated size of the difference in reactivity between type A and $B$ subjects appear to be so small, what other explanations could there be for the low consistency in the experimental results? We think that methodological reasons will have to be considered too. In other words, the validity of the experimental results will have to be examined in more detail.

\section{Validity of the experiments on reactivity}

Barrett (1988) points out in a humorous, but nonetheless disturbing, article that comparing two smiall groups of subjects may lead to highly variable outcomes, ranging from highly significant differences to insignificant ones while in fact a moderate effect is present in the population. Such highly varying outcomes may be expected when insufficient power is present in the experiments used. If one regards the outcome of an experiment as an estimate of the population effect, such an outcome is part of a distribution of outcomes from similar experiments with the population effect as a mean. In a low power experiment only the large effects part of the distribution will lead to significant results and only a minority of the possible outcomes will lead to significant results, while the majority of the outcomes leads to insignificant results. Instead of being able to conclude that a moderate effect is to be expected, as one would have done when several small studies had been combined to one large study that had more power, one 
is left with a confusing collection of apparently contradicting findings. Thus, it is important to take power into consideration when discussing the implications of insignificant results for the theory that was tested.

The risk of insufficient power is especially pertinent for reactivity experiments since, due to the increased refinement of the general hypothesis, most experimenters have included in their experiments an increasingly large number of tasks, often using separate subgroups of subjects for each task, thereby reducing the number of subjects performing each task. Furthermore, because different types of response patterns may be found, depending on the type of task used as a stressor, an increasingly large number of dependent variables have been measured, from which an increasingly large number of physiological indices have been obtained. This, in turn, has resulted in an increasingly large number of F-tests. When so many test have been done the risk of a sizeable number of experimental findings that are based on incidental significant findings also increases.

How likely is it that the majority of the published positive results on reactivity are based on type I errors (i.e. significant but accidental results that happen to fit the ruling hypothesis) and that the negative results are based on type II errors (i.e. that in spite of the insignificant test a difference in reactivity does exist)?

In order to answer this question we will first describe the factors that influence the likelihood of these two types of errors occurring in more detail. Then the impact of these factors on the accumulated experimental evidence for greater reactivity in type A subjects will be discussed.

\subsubsection{The probability of type 1 and type II errors}

The probability of making a type I error is dependent on the level of significance, $a$, that has been chosen. Once the value of a has been set, the probability of a type II error is dependent on the number of subjects employed, the efficiency of an experimental design, the expected difference between the two groups of subjects, and the probability of making a type I error.

According to Fleiss (1986) sample size estimations can be calculated given a specific level of alpha and of beta, the size of the expected difference between two groups of subjects, and the variance. This can be done according to equation 4.1.

$$
n=\frac{2 \sigma^{2}\left(Z_{a / 2}+Z_{B}\right)^{2}}{\left(\mu_{A}-\mu_{B}\right)^{2}}
$$

In this equation, $n$ is the required number of subjects in each of the two groups, $\mu_{\mathbb{A}}$ and $\mu_{B}$ are the mean scores in group $A$ and $B_{;} ; \sigma$ is the standard deviation of the scores on which the two groups are compared. The standard deviation is assumed to be equal in both groups. $Z_{\alpha / 2}$ and $Z_{\beta}$ are the Z-values belonging to the critical levels chosen for $a$ (the probability of making a type I error) and $\mathbb{B}$ (the probability of making a type II error).

The equation has been proposed to calculate the number of subjects needed to be able 
to detect a certain difference before an experiment is conducted. In doing so, one has the advantage that the risk of the two types of error can be decided beforehand. However, the same equation can also be used to calculate the probability of making a type II error of a particular experiment once it has been executed. In order to calculate this probability the equation 4.1 may be written as

$$
z_{\beta}=\sqrt{\frac{n}{2}} \times \sqrt{\frac{\left(\mu_{\mathrm{a}}-\mu_{\beta}\right)^{2}}{\sigma^{2}}}-z_{\mathrm{a} / 2}
$$

When an analysis with repeated measurements or an analysis of covariance has been used in the experiment, which is usually the case in reactivity experiments, the efficiency of the experimental design increases. The increase in efficiency is due to the fact that in these types of analysis differences in responses are compared instead of differences in stress level. When during baseline conditions no differences exist between the two groups that are compared, the difference in stress level between the two groups will be equall to the difference in change from baseline to stress: the stress response. The variance of the stress responses, however, will be smaller than that of the stress level scores. In case an analysis with repeated measures has been used, the reduction is $2\left(1-r_{b s}\right)$. When an analysis of covariance has been used, the reduction is $\left(1-r_{b s}^{2}\right) . r_{b s}$ is the correlation between the two measurements used in the repeated design ot in the analysis of covariance. In the case of reactivity experiments these are the measurements obtained during the baseline and during the stress tasks.

In order to calculate the probability of making a type II error when these types of analysis have been used, equation 4.2 may be replaced by equation 4.3 , when repeated measures have been included, or by equation 4.4 when an analysis of covariance has been used.

$$
\begin{gathered}
Z_{\beta}=\sqrt{\frac{n}{2}} \times \sqrt{\frac{\left(\mu_{\alpha}-\mu_{\beta}\right)^{2}}{2\left(1-\mu_{b s}\right)^{2}}}-Z_{\alpha / 2} \\
Z_{\beta}=\sqrt{\frac{n}{2}} \times \sqrt{\frac{\left(\mu_{\alpha}-\mu_{\beta}\right)^{2}}{\left(1-\Gamma_{b a}^{2} \sigma^{2}\right.}}-Z_{\alpha / 2}
\end{gathered}
$$

Since $\sqrt{ }\left(2\left(1-r_{b s}\right) \sigma^{2}\right)$ is equivalent to $\sigma_{\text {response, }}$, the square root of the response variance, and $\checkmark\left(\left(1-r_{b B}^{2}\right) \sigma^{2}\right)$ is equivalent to $\sigma_{\text {residual }}$ the square root of the residual variance, and since $\mu_{A}-\mu_{B}$ is the same as $\mu \delta_{A}-\mu \delta_{B}$, assuming that no difference exists between type $A$ and $B$ subjects during baseline, these equations can also be written as 


$$
\begin{aligned}
& Z_{\beta}=\sqrt{\frac{n}{2}} \times \frac{\left(\mu \delta_{A}-\mu \delta_{d}\right)}{0_{n p o n}}-Z_{\alpha / 2} \\
& Z_{\beta}=\sqrt{\frac{n}{2}} \times \frac{\left(\mu \delta_{A}-\mu \delta_{B}\right)}{\sigma_{\text {nalodar }}}-Z_{\alpha / 2}
\end{aligned}
$$

After all these transformations of the basic formula to calculate the required sample size into the equations 4.5 and 4.6 , it is evident that in order to calculate the Z-value of beta for a particular experiment one needs to know the number of subjects in each group, the size of the expected difference in response between two groups, the standard deviation of the responses or the square root of the residual variance in both groups, and one has to decide on the risk of a type I error one wants to accept.

In the next section we will describe how, using meta-analytic techniques, a good approximation of the expected difference in response between the two groups, expressed as a fraction of either the response variance or the residual variance, may be obtained. When the expected effect size is calculated, we will be able to see whether or not adequate power was present in the experiments on reactivity differences between type $\mathrm{A}$ and $\mathrm{B}$ subject. The meta-analysis also enables us to formally test the ideas that were presented by the reviewers regarding the influence of the classification instruments and the tasks used as stressors.

\subsubsection{A meta-analysis of experiments on reactivity}

A meta-analysis was performed to answer two questions. First what is the expected size of the difference in stress response between type $A$ and $B$ subjects, and second is the size of this difference affected by the type of classification instrument and or the type of stressor.

The analysis was performed on the results of experiments similar to those selected by Harbin (1989) for his meta-analysis. Harbin used the following selection criteria. All selected studies had to have appeared as doctoral dissertations or as articles in refereed journals. Either the JAS or the SI had to have been used to assess type A behavior. One or more of the following dependent variables had to have been included in the study: heart rate, systolic blood pressure or diastolic blood pressure. Subjects had to be students or adults. Finally, stressors of a psychological nature had to have been employed to elicit physiological activation.

Our selection of the articles differed in two ways. First, we restricted the selection of articles to studies on males. Second, we omitted those articles that had not appeared in journals, since quite often studies described in dissertations later appear in journals too, causing them to be entered in analysis twice. In the reference list the selected artical for the meta-analysis are marked with 1 and/or 2 .

Beside the difference in the selection of the experiments, our meta-analysis differed in two additional ways from the one used by Harbin. First, Harbin used the combined 
significance level of the difference in response between type A and B subjects. Levels of significance obtained in any experiment are dependent on the size of the effect in the sample of subjects in the study, and on the sensitivity of the experiment. The latter is dependent on the number of subjects used, and on some other design factors like whether repeated measurements were used and whether baseline measurements and stress measurements were made within a short or a long time span. Since experiments vary in this respect a high level of significance may be found in a highly sensitive experiment, when only a small difference between groups is present, or in a less sensitive experiment, requiring a moderate or large effect. Therefore, since a transformation of combined probabillities into a combined effect size is not possible and since we were interested in the size of the effect, we investigated an indicator of the size of the difference in response between type A and B subjects. Second; the selection of the actual tests of the difference in reactivity between type $\mathbf{A}$ and $\mathbf{B}$ subjects was different. Whenever several tests were made, based on the same set of subjects, one test was randomly selected in order to assure a set of independent tests.

According to the methods employed by Rosenthal (1984), a Hedges's $g$ score was calculated for each of the tests that investigated the effect of type A behavior on the size of the stressor response. Hedges's $g$ is an estimate of the population effect size and it is defined as the difference between two groups on a certain variable, divided by the standard deviation of that variable, pooled over both groups, as presented in equation 4.7 . In our analysis the yariable was the mean response from base to stress $(M \delta)$ and the two groups were type A and B subjects.

$$
\text { Hedges's } g=\frac{M 8_{A}-M 8_{B}}{S \delta_{A+B}}
$$

The scores were calculated on the basis of the reported $F$ or $t$ values of the tests of a difference in reactivity between type $A$ and $B$ subjects or the $r$ value of the correlation between behavioral type and degree of reactivity. If these values were lacking, the reported $\mathrm{p}$ values were used, in combination with the degrees of freedom, to reconstruct the $t$ value. Transformation formulas for both $F, t$, and $r$ values into Hedges's $g$ were found in Rosenthal (1984). They are presented in Equations 4.8 to 4.10.

$$
\begin{aligned}
& \text { Hodgos'g }=\sqrt{F} \times \sqrt{\frac{1}{n_{A}}+\frac{1}{n_{B}}} \\
& \text { Hodgos' } g=t \times \sqrt{\frac{1}{n_{A}}+\frac{1}{n_{B}}}
\end{aligned}
$$




$$
\text { Hedges's } g=\frac{r}{\sqrt{\left(1-r^{2}\right)}} \times \sqrt{\frac{\left(n_{A}+n_{B}-2\right)\left(n_{A}+n_{B}\right)}{n_{A} n_{B}}}
$$

In the equations $n_{\mathrm{A}}$ and $n_{\mathrm{B}}$ are the number of type $\mathrm{A}$ and type $\mathrm{B}$ subjects.

When all these values were lacking, tables or figures were inspected to find the absolute difference in response between type $A$ and $B$ subjects. These differences were then converted to Hedges's g scores, using equation 4.7, using an estimated standard deviation consisting of the mean of the standard deviations that were found in the other experiments. (systolic pressure response: mean sd $=8.3 \mathrm{mmHg}$; diastolic pressure response: mean sd $=5.2 \mathrm{mmHg}$, heart rate response: mean sd $=6.6 \mathrm{beats} / \mathrm{min}$ ) When no indication of the size of a difference in response was given and no test statistics were available, a Hedges's $g$ score of 0 was used.

The scattergram of the resulting g-scores as a function of the number of subjects used in each test is given in figure 4.3.1. It can be clearly observed that the obtained estimate of the size of the difference in reactivity between type $A$ and $B$ subjects varied more when fewer subjects were employed in an experiment. Before calculating mean $g$ values or comparing Hedges's $\mathrm{g}$ values of experiments belonging to different subsets, a correction is necessary that takes this phenomenon into account. Rosenthal (1984) advises the reciprocal of the variance of the responses made in each experiment as weighing factor for each $\mathrm{g}$. The formula for this weighing factor, $w_{\mathrm{j}}$, is given in equation 4.11 in which $n_{\mathrm{A}}$ and $n_{\mathrm{B}}$ are the number of type $\mathrm{A}$ and of type $\mathrm{B}$ subjects, and $F$ is the Fralue obtained when testing the difference in stress response between type $\mathrm{A}$ and $\mathrm{B}$ subjects.

$$
w_{I}=\frac{2\left(n_{A} n_{B}\right)\left(n_{A}+n_{B}-2\right)}{\left(n_{A}+n_{B}\right)\left(F+2\left(n_{A}+n_{B}-2\right)\right)}
$$

Weighted mean $g^{\prime} s\left(g_{W}\right)$ were calculated for all studies combined and for each of the subsets using equation 4.12 .

$$
g_{w}=\frac{\sum w g_{j}}{\sum w_{f}}
$$

To test if these weighted mean $\mathrm{g}^{\prime}$ s indicated a significant difference in response between type $A$ and $B$ subjects the standard normal deviate, $Z$, was used. This was done using equation 4.13 , in which $g_{w}$ stands for the weighted mean $g$, and $\Sigma w_{\mathrm{f}}$ is the sum of the wrelghts used in the studies on which the weighted mean $\mathrm{g}$ was based.

$$
z=g_{w} \times \sqrt{\sum w_{j}}
$$



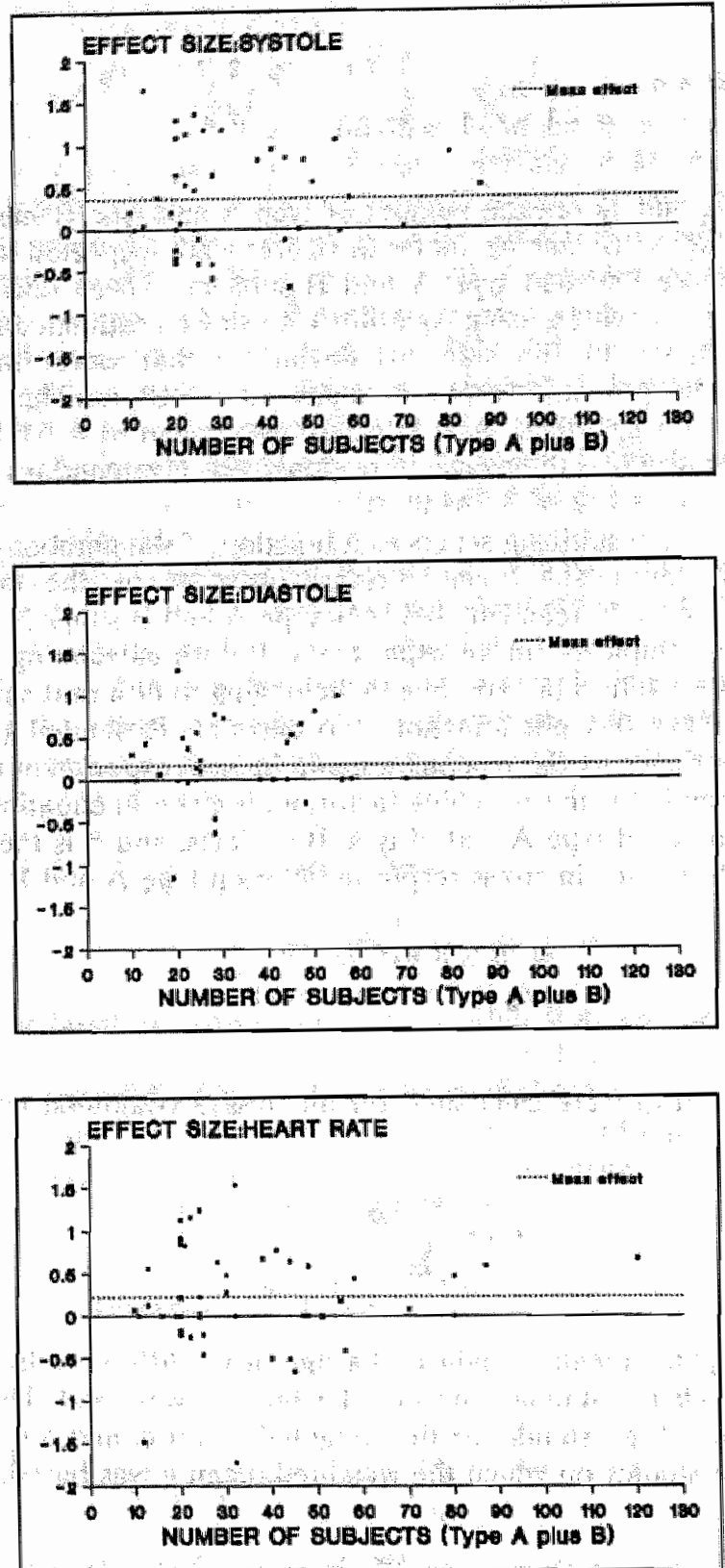

Figure 4.3.1: Relationship between estimated effect size and number of subjects. 
The overall weighted mean g values of the three variables used in the meta-analysis are presented in table 4.3.1.

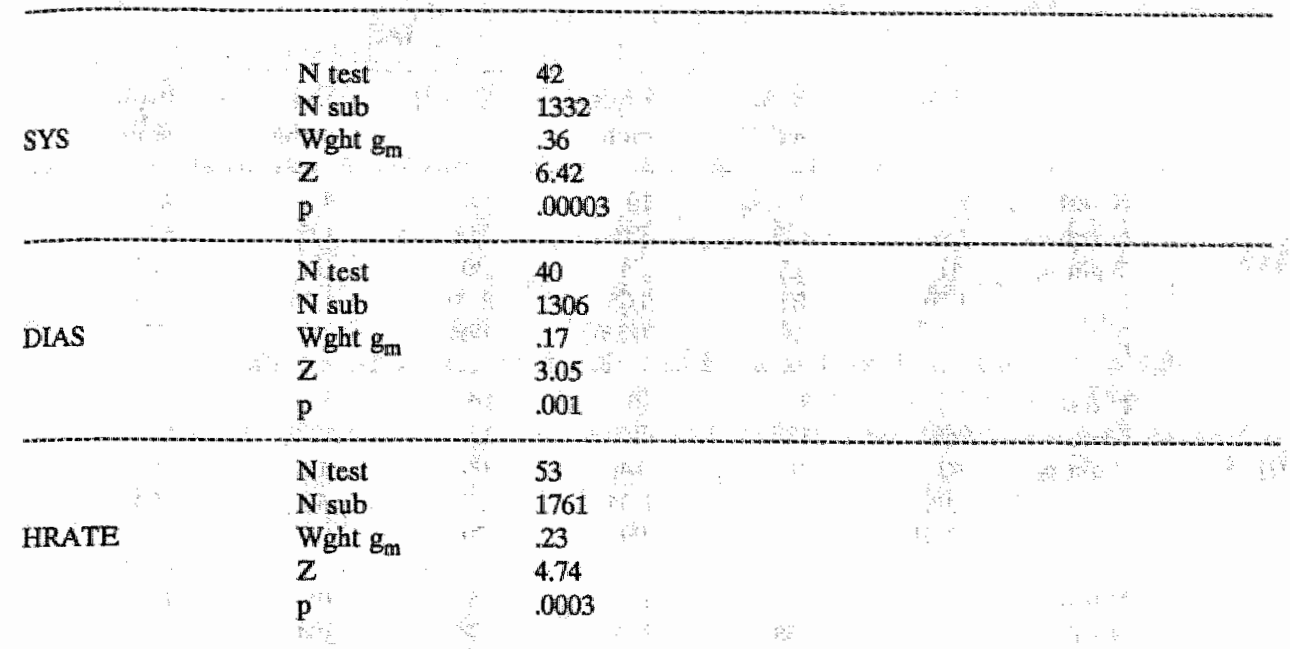

Table 4.3.1: Welghted mean $g$ values calculated over all experiments ( $N$ test is number of experimental tests; $N$ sub is total number of subjects involved in the different tests; $W g h t g_{m}$ is weighted mean of the difference in reactivity botween type $A$ and $B$ subjects; $Z$ is the value indlcating the level of significance of the difference in reactivity and $p$ is the $p$ walue belonging to the $Z$-score).

It can be seen in table 4.3 .1 that the overall size of the difference in reactivity, on all three variables, is rather small, but clearly significant.

In order to answer the second question, six subsets of studies were distinguished to see whether the effect size was indeed influenced by the way in which the subjects were selected and/or by the type of stressor they were exposed to. The classification of the subsets was done as follows: first, a distinction was made between studies in which the SI was used to classify type A and B subjects and those in which the JAS was used as a classification instrument. Then, within these two main categories, three types of task were distinguished. The first type consisted of psychomotor tasks, in which the subjects had to push reaction buttons or to manipulate joy-sticks or a diall. The second type consisted of non-verbal cognitive tasks, like serial subtraction tasks in which the subjects had to report the outcome only at the and of the task. The third type consisted of verbal tasks. Using these two subdivisions, a total of six subsets of studies were formed. In table 4.3 .2 the results of the analyses for each of the subsets are presented. In this table it is shown that the size of the difference varies among the subsets from virtually zero to half a standard deviation. In order to see whether the size of the effect was indeed influenced by the way in which the subjects were selected and/or by the type of stressors they were exposed to, or whether the variation in size occurred just by chance, first a test for heterogeneity was performed for each of the three variables used in the meta-analysis i.e. systolic blood 
pressure, diastolic blood pressure and heart rate.

\$I

JAS

\begin{tabular}{|c|c|c|c|c|c|c|c|}
\hline & & Verbal & $\begin{array}{l}\text { Non- } \\
\text { verbal }\end{array}$ & $\begin{array}{l}\text { Psycho- } \\
\text { motor }\end{array}$ & Verbal & $\begin{array}{l}\text { Non } \\
\text { werbal }\end{array}$ & $\begin{array}{l}\text { Psycho- } \\
\text { motor }\end{array}$ \\
\hline SYS & $\begin{array}{l}\text { Nest } \\
\text { N sub } \\
\text { Wght g } \\
\text { Z } \\
\text { p }\end{array}$ & $\begin{array}{l}8 \\
268 \\
.41 \\
3.39 \\
.0005\end{array}$ & $\begin{array}{l}2 \\
138 \\
.15 \\
.88 \\
19\end{array}$ & $\begin{array}{l}10 \\
383 \\
54 \\
5.05 \\
00000\end{array}$ & $\begin{array}{l}15 \\
3.48 \\
.30 \\
2.69 \\
.004\end{array}$ & $\begin{array}{c}4 \\
128 \\
35 \\
194 \\
.03\end{array}$ & $\begin{array}{l}2 \\
67 \\
-.03 \\
-.12 \\
.55\end{array}$ \\
\hline DLAS & $\begin{array}{l}\text { N test } \\
\text { Nowb } \\
\text { Wght g } \\
Z \\
\text { p }\end{array}$ & $\begin{array}{l}8 \\
268 \\
.47 \\
3.94 \\
.0001\end{array}$ & $\begin{array}{l}2 \\
138 \\
.0 \\
.0 \\
.50\end{array}$ & $\begin{array}{l}10 \\
383 \\
.14 \\
1.34 \\
.09\end{array}$ & $\begin{array}{l}14 \\
322 \\
.06 \\
53 \\
.30\end{array}$ & $\begin{array}{c}4 \\
128 \\
12 \\
66 \\
26\end{array}$ & $\begin{array}{l}2 \\
67 \\
-.01 \\
-.04 \\
-.52\end{array}$ \\
\hline HARATE & $\begin{array}{l}\text { N test } \\
\text { N sub } \\
\text { Wght } \mathrm{g}_{\mathrm{s}} \\
\mathrm{Z} \\
\mathrm{p}\end{array}$ & $\begin{array}{l}8 \\
248 \\
.19 \\
1.56 \\
.06\end{array}$ & $\begin{array}{l}2 \\
138 \\
.18 \\
1.05 \\
.15\end{array}$ & $\begin{array}{l}10 \\
383 \\
.35 \\
3.31 \\
.0006\end{array}$ & $\begin{array}{l}16 \\
374 \\
.18 \\
1.68 \\
.05\end{array}$ & $\begin{array}{c}10 \\
304 \\
.04 \\
34 \\
38\end{array}$ & $\begin{array}{l}7 \\
314 \\
.37 \\
3.18 \\
.001\end{array}$ \\
\hline
\end{tabular}

Table 4.3.2: Results of meta-analysis of experiments testing a difference In reactility between type $A$ and $B$ subjects ( $N$ test is number of experimental tests; $N$ sub is total number of subjects involved in the different tests; Wigt $g_{s}$ is weighted mean of the difference in reactivity batween type $A$ and $B$ sublects of a particular subset; $Z$ is the standard normal deviate of the combined difference in reactivity: $p$ is the $p$ value belonging to the $Z$ score; SYS is systollo bload pressure, DIAS is dlastollo blood pressure; HRATE is heart rate.)

Using equation 4.14 a weighing factor $\left(w_{s}\right)$ was calculated for each subset, with the weighing factor $\left(w_{1}\right)$ of each of the studies that belonged to the subset.

$$
w_{s}=\sum w_{i}
$$

Using equation 4.15 , this weighing factor, together with the weighted mean $\mathrm{g}$ of each subset $\left(g_{\mathrm{s}}\right)$ and the overall weighted mean $\mathrm{g}\left(g_{\mathrm{m}}\right)$, was used to calculate the Chi

$$
x^{2}=\sum w_{s}\left(g_{s}-g_{m}\right)^{2}
$$


indicating whether or not the weighted mean $\mathrm{g}^{3}$ s of the six subsets differed significantly among themselves (Rosenthal 1984, p80). The heterogeneity of systolic pressure was found to be significant $\left(\mathrm{Chi}^{2}=14.0, \mathrm{df}=5 \mathrm{p}<.02\right)$, meaning that the weighted mean $\mathrm{g}$ 's of each subset do vary. The heterogeneity of diastolic pressure (Chi ${ }^{2}=8.95$, $\mathrm{df}^{2}=5$ $.10<\mathrm{p}<.20)$ and heart rate $\left(\mathrm{Chi}^{2}=5.75, \mathrm{df}=5 \mathrm{p}<.30\right)$ were not significant, meaning that the variation in the size of the weighted mean g's among the six subsets is more likely due to chance.

On the basis of the significant heterogeneity test a follow-up analysis was made for systolic blood pressure to investigate whether the type of classification instrument and/or the type of task influenced the size of the Hedges's g of the subsets. It should be noted that the three categories of tasks are not equally distributed over the two larger subsets of experiments based on the classification instruments. For instance, when blood pressure is used as a dependent variable, the number of tests that made up the JAS/psychomotor subset is much smaller than the number of tests in the SI/psychomotor subset.

To avoid the risk of confounding task effects and classification instrument effects we first analyzed the difference between the two classification instruments separately for each type of task, using equation 4.16 .

$$
z_{d / f F}=\frac{g_{S I}-g_{J A S}}{\sqrt{\frac{1}{w_{S I}}+\frac{1}{w_{J A S}}}}
$$

In this equation, $g_{\mathrm{SI}}$ and $g_{\mathrm{IAS}}$ are the weighted mean g's belonging to the subset of studies that used the same type of tasks but in which the subjects were selected either by the SI or by the JAS. $w_{\mathrm{SI}}$ and $w_{\mathrm{JAS}}$ are the weighing factors that belong to the respective subsets. These weighing factors were calculated using equation 4.14.

Then we combined these three test according to the method of the added Z-scores (Rosenthal 1984, p94), using equation 4.17,

$$
z_{t}=\frac{\sum z_{1}}{\sqrt{n_{z}}}
$$

in which $Z_{\mathrm{j}}$ stands for the $Z$-value of the result of one of the difference tests and $n_{\mathrm{Z}}$ is the number of tests on which the combined Z-score is based. The results are shown in table 4.3.3. No evidence was found of over-all superiority of the SI as an instrument for predicting larger reactivity in type A subject. The combined differences in size of the effect between the SI and the JAS did not reach an acceptable level of significance. However, systolic blood pressure was higher in SI defined subjects when psychomotor tasks were used ( $\mathrm{p}<.04$ two-sided). A similar procedure was followed when analyzing the effects of type of task. 


\begin{tabular}{|c|c|c|c|}
\hline Tasks & $g_{\text {JAS }}$ & SII & $z_{\text {dilifi }}$ \\
\hline verlad & 30 & 41 & 67 \\
\hline non-verbal & 35 & 15 & $-80^{\circ}$ \\
\hline Peycho motar & .03 & 54 & $214^{*}$ \\
\hline
\end{tabular}

combined $\left(\mathcal{Z}_{\text {add }}\right)$

Table 4.3.3. Differonces in weighted mean $g$ of sysitollc blood pressure between JAS-defined subjects and Sl-deflined subjects. ${ }^{*}=0<04, g_{\text {JAS }}$ ls the welghted mean g value besed on experiments using the JAS as classiffcatlon instrument, $g_{5}$ is the weighted mean $g$ value based on experiments using the SI as classification instrument, $Z_{\text {diff }}$ is the $Z$-value of the difference between the $g_{j A S}$ and the $g_{S p} Z_{\text {add }}$ is the $Z$ value of the combined $Z_{\text {ofif }}$ values).

Tasks were compared separately for each type of classification instrument and the two resulting tests for each comparison were then combined. The results of these analyses are presented in table 4.3.4.

\begin{tabular}{|c|c|c|c|}
\hline Classification & Ewerb & $\mathrm{g}_{\mathrm{n} \text {-vert }}$ & $z_{\text {diff }}$ \\
\hline JAS & 30 & 35 & -.24 \\
\hline SI & .41 & 15 & 1,24 \\
\hline \multicolumn{4}{|l|}{ Combined $\left(Z_{\text {add }}\right)$} \\
\hline Classifification & geerb & $\mathrm{g}_{\mathrm{psy} \text {-mot }}$ & $z_{\text {diff }}$ \\
\hline JAS & 30 & -.03 & 1.23 \\
\hline SI & 41 & .54 & -.81 \\
\hline \multicolumn{3}{|l|}{ Combined $\left(Z_{\text {add }}\right)$} & 30 \\
\hline Classification & Bn-verste & $\mathrm{g}_{\text {psytmot }}$ & $z_{\text {diff }}$ \\
\hline JAS & 35 & .03 & -1.25 \\
\hline SI & 15 & 34 & 1.93 \\
\hline
\end{tabular}

Combined $\left(\mathbb{Z}_{\text {add }}\right)$

Table 4.3.4 Differences in welghted mean g of systolic blood pressure between the three types of tasks. ( $Q_{\mathrm{u} \text { wib }}$ is the welghted mean $g$ of the experiments using verbal tasks; $g_{n \text {-verb }}$ is the welghted mean $g$ of the experiments using non-verbal tasks; $g_{p s y \text { mot }}$ is the weighted mean $g$ of the experiments using psycho-motor tasks; $Z_{\text {diff }}$ is the $Z$-value of the difference berween the $g$ 's of the different task conditions; $Z_{\text {add }}$ is the $Z$-value of the combined $Z_{\text {diff }}$ values). 
None of the comparisons between the tasks resulted in a significant difference, indicating that no robust differences exist in the extend to which these three types of tasks elicit differences in response between type A and type B subjects.

The answers to the two questions, what is the expected size of the difference in stress response between type $A$ and $B$ subjects and is the size of this difference affected by the type of classification instrument and/or the type of stressor are as follows:

Expected size of the difference. Since the tests of heterogeneity of diastolic pressure and heart rate were not significant, the best estimate for the expected size of the difference of these variables can be found by looking at the overall mean, as presented in table 4,3.1 for diastolic blood pressure a $\mathrm{g}$ of .17 and for heart rate $\mathrm{g}$ of .23 may be expected. The test of heterogeneity of systolic blood pressure has been found to be significant, so the overall mean is a not the most appropriate estimate of the difference in systolic blood pressure reactivity between type A and B subjects. $A$ better estimate can be found under the appropriate classification instrument by task subset as presented in table $4.3 .2 \mathrm{a} \mathrm{a} \mathrm{g}$ varying between -.03 and .54 can be expected on this variable. In absolute values type A subjects will have a 1 to $4 \mathrm{mmHg}$ larger reaction in systolic blood pressure, a $.88 \mathrm{mmHg}$ larger reaction in diastolic blood pressure and 1.5 beats larger increase in heart rate than type B subjects.

Effect of task and/or classification method. The differences between the weighted mean $\mathrm{g}$ values of the various subsets of studies are only rarely significant. This means that the claims made in the various review articles about the SI being the first choice as a classification instrument are based on only suggestive, but not very substantial, evidence. The same is true for the recommendations of the psychomotor tasks as the most appropriate stressor. In one instance an interaction between classification and task characteristic was observed: the combined use of psychomotor task and the SI as a selection instrument resulted in a somewhat larger difference in systolic blood pressure response between type $A$ and $B$ subjects but only in one of the three contrasts in which this combination was used did it result in a significant difference with an other subset. Regarding the other two dependent variables, the absence of a significant test of heterogeneity makes it doubtful that robust effects of classification and/or task characteristics on diastolic blood pressure and heart rate exist.

This implies that, in order to explain the variations in the experimental results, one must look for other factors like the possible lack of power, or the variability of power between and/or within experiments.

4.3.2.3 The power of the tests of a difference in reactivity between type $A$ and $B$ subjects

In section 4.3.2.1 it was shown that the power of an experimental test can be calculated when one knows the number of subjects in each group, the size of the expected difference in response between two groups, the standard deviation of the responses or the square root of the residual variance in both groups, and has decided on the risk of a type I error one wants to accept. It should be noted that the middle term of equation 4.2 is equivalent 
to the population effect size which can be estimated by calculating the weighted mean Hedges's $\mathrm{g}$ from similar experiments.

With the number of subjects we used and the estimated size of the difference in heart rate response based on the meta-analysis, this means that the power of our experimental design to find the expected difference in response on heart rate was only $14 \%$ in the first experiment and $16 \%$ in the second experiment.

Comparing our results with those of the other experiments used in the meta-analysis, it appears that the power in our experiments was representative of what is generally found. We used 28 subjects in experiment I and 24 in experiment. II. The mean number of subjects used in the other studies was 33 with a range of $10-120$. This means that the power to detect differences in heart rate responses varied from $8 \%$ to $50 \%$ with a mean of $17 \%$.

When comparing the size of the difference in reactivity that can be detected with the size of the difference that may be expected one cannot fail to notice the large discrepancy between the two. The power of the average experiment is simply too low to allow the expected difference to be detected in the majority of the cases. With a risk of type II errors as large as $80 \%$ inconsistency in the experimental results may be expected.

Another explanation for the large degree of inconsistency in experimental results may be a difference in power between or within experiments. We have shown in section 4.3.2.1 that, given a certain level of $\alpha$, power is determined by number of subjects and the population Hedges's g. One may remember that the size of Hedges's $\mathrm{g}$ is determined by two factors: the obtained difference in response and the variance of the response or, When an analysis of covariance was used, the residual variance. The standard deviation of the response and the square root of the residual variance are dependent on the standard deviation of the stress level scores and the size of the correlation between baseline values and stress values. These factors are, in turn, determined partly by chance related sample characteristics and partly by systematic, experiment related factors. For instance the correlation between baseline and stress level values is influenced by measurement accuracy, which may well differ between the various dependent variables, and by the time span between baseline and stress measurement. As long as systematic differences like these are not controlled for or at least measured, one is uncertain whether the a priori chance of getting significant results is equal for all tests.

\subsubsection{Number of F-tests employed}

In order to get some idea of how often these tests have been employed, the exact number of F-tests performed in the first experiment of this thesis was counted. A distinction was made between those F-test performed to test the hypotheses, basically interactions between group and condition or differences in overall trend between the groups, and those that were used in follow-up analyses. These follow-up analyses consisted of checking whether: (1) the experimental manipulations did, indeed, have the expected general effects; (2) there were main group effects; (3) the groups differed during tasks or during baseline; (4) there were differences in the orthogonal trend components between the groups. Moreover, higher order interactions that might explain the absence of lower order effects have been inspected, leading to further tests. 
Two different analysis models were used in the first experiment. The first one was used to test the hypothesis of the overall difference in reactivity, and of some of the predictions of the saliency hypothesis like the effects of feedback and of the anticipation and the performance of the venous puncture. The second model was used for analyses of the effects of time pressure, both during tasks and during the rest periods. The first analysis model resulted in two direct F-tests for each dependent variable - one for difference in reactivity related to $\mathrm{AB}$ typology and one for differences in reactivity related to level of exhaustion - and eleven follow-up F-tests. Three of the latter dealt exclusively with $\mathrm{AB}$ typology, three with level of exhaustion, four with interactions between the two group factors and one with the main condition effect. The second analysis model also results in two direct $F$-tests for each dependent variable, one for difference in reactivity related to $A B$ typology and one for differences in reactivity related to level of exhaustion. Twenty one F-tests were used for follow-up analyses. Five dealt exclusively with $A B$ typology, five with level of exhaustion, six with interactions between the two group factors, four with third order interactions between trend components and the two group factors and one with the main trend effect. In table 4.3.5 the resulting number of F-tests that were employed in testing differences in reactivity involving $\mathbf{A B}$ typology are presented. From this table it can be seen that, in the first experiment, the hypothesis of greater reactivity in type A subjects was tested eight times for each of the dependent variables resulting in a total of 46 tests.

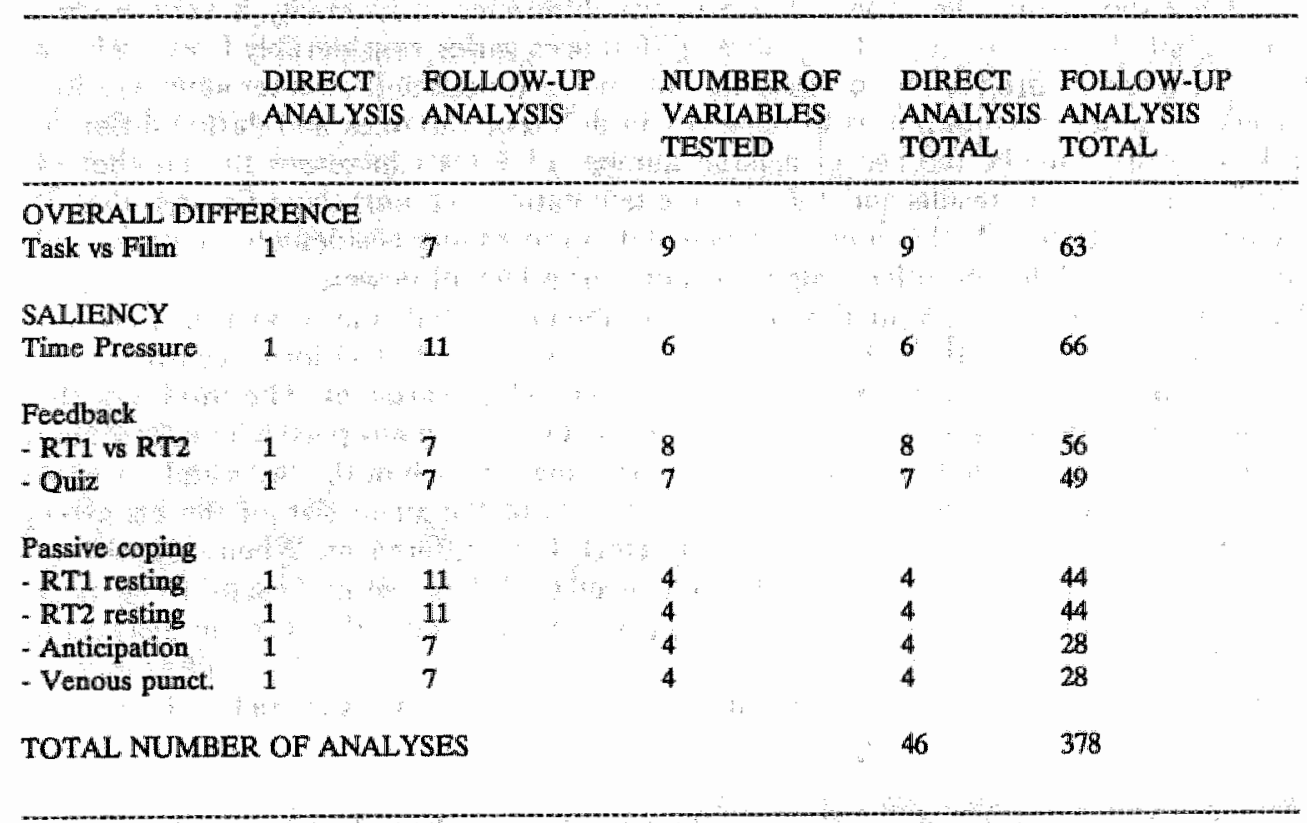

Table 4.3.5: Number of F-tests employed in the analyses of differences in reactivity between type $A$ and $B$ subjects in chapter two of this thesis. 
Furthermore, no less than 108 follow-up tests have been employed for each dependent variable, of which 68 involved AB typology in some way. This brought the total number of follow-up tests to the staggering number of 686,378 inwolving $A B$ typology. Of course one must tealize that the dependent variables are, to a certain extent the reflection of the same underlying physiological processes. Furthermore, if the hypothesis of a general larger reactivity in type $A$ subjects is correct, significant findings will be found in more than one experimental condition. Stated differently, neither the different variables nor the different conditions are independent. Unfortunately, we have no means to assess the degree of dependency between the various tests. Therefore, the number of significant $F$ tests that can be expected on the basis of pure chance cannot be estimated and be used as a correction for the obtained number of significant F-tests.

The number of tests in just one experiment is very large particularly when not only the direct tests of the hypothesis of larger reactivity in type A subjects but also others involving either the type A behavior pattern (or another subdivision in high and low risk groups) are included.

\subsubsection{Consequences of methodological problems}

From the power calculations it becomes obvious that if we trust the results of the metaanalysis, our experiments, like most of the others, had a poor chance of finding clearly interpretable results; the risk of type II errors was around $80 \%$ ! Furthermore, it has not only been shown that the expected size of the difference in reactivity is very modest indeed, but also the ability to detect these differences varies considerably from variable to variable and from situation to situation. This makes it doubtfull whether equal a priori chances of detecting differences in reactivity in different variables and during different tasks do exist. Finally, the use of a large number of F-tests increases the number of accidental significant results and, thereby, the temptation to report those findings that fit the hypothesis tested. At the same time, actual differences may accidentally go unnoticed due to chance-related negative tests results and/or a lack of power.

The consequence of this methodological problem is that the evidence thus far accumulated is not sufficient to be able to make sound decisions regarding the appropriate choices of stimulus conditions and dependent variables. The results of the various experiments might well be explained by the fact that in any particular experiment the a priori chance of finding an effect is not the same for each of the tasks and for each of the variables used, due to the cumulative effects of the small size of the expected differences and to variation in the power to detect these differences. When inconsistencies in the experimental results are partly influenced by differences in the a priori chances of finding a significant effect, an explanation of the observed differences in activation pattern in basic physiological terms, becomes very difficult indeed. This unfortunately hampers the right choice of experimental situations and of dependent variables for future experiments.

Most of the problems discussed above in the context of the type A behavior pattern are also encountered when trying to establish differences in stress reactivity between vital and exhausted subjects. 
Instead of walking away in despair from this area of research, we will now suggest measures for dealing with the three following problems: 1) the inadequate power of the experiments, 2) the variability in power from situation to situation and from dependent variablle to dependent variable within a particular experiment and, 3) the large number of F-tests used.

\subsubsection{Increase in the power of the experiment}

\subsubsection{Increase in the expected difference in reactivity}

This problem is probably the most difficult one since we just have shown that the solutions that have thus far been suggested, such as using the SI to classify type A and $\mathrm{B}$ subjects, using hostility scores instead of overall $\mathrm{AB}$ typology, or measuring only those physiological variables that reflect beta-sympathetic activation, are not based on reliable findings of previous experiments. The recommendation of giving the subjects 'moderately difficult' tasks is not very helpful either since clear and unambiguous decisions, based on previous research, regarding which tasks are moderately difficult are not possible. Since experimental data cannot help us, we might look to the epidemiological studies to see if they suggest some solution. First let us consider the way in which most of the reasoning based on the epidemiological results probably has gone: type A subjects have a risk of getting a myocardial infarction that is twice as large as type B subjects. This means that type A subjects have twice the proportion of future cases vs controls as type B subjects. If hyperreactivity plays an important role in getting them into trouble one may assume that the same relation will be found for the proportion of high vs low reactors as for future cases vs non-cases. If one does accept this assumption, how easy is it than to demonstrate a difference in reactivity?

An answer to this question can be obtained by a transformation of the odds ratio of 2 for type A subjects, regarding myocardial infarction, in the expected difference between the two groups in mean reactivity scores. The proportion of high reactors and low reactors is assumed to be similar to those of future cases vs non cases. For a five year observation period this might be set at $5 \%$ cases vs $95 \%$ non-cases. High reactors are defined as giving a reaction in the upper $5 \%$ of the reactions of all the subjects and low reactors as giving a reaction below the upper $5 \%$. In table 4.4.1 the proportion of high and low reactors within type A and type B subjects is given which result in an odds ratio of 2 . This can also be depicted in two normal function curves, one for type A subjects and one for type B subjects, that are positioned in such a way that mean of the curve of the type $A$ subjects is shifted towards the high reactivity side in such a way that $6 \%$ of its area is to the right of the $5 \%$ limit of all subjects while for the B subject only $3 \%$ is right of the $5 \%$ limit of all subjects. This corresponds to a difference in Z-scores of 1.87 minus 1.55 which is .32. Thus, the expected difference in the means of type A and type B subjects, assuming a one-to-one relationship between risk of $M I$ and belonging to the group of high-reactors, is 32 standard deviation. That this assumption is not violated in a serious way can be demonstrated by the fact that the size of the difference in reactivity calcullated on the 


\begin{tabular}{lll} 
& $\begin{array}{l}\text { High } \\
\text { reactor }\end{array}$ & $\begin{array}{l}\text { Low } \\
\text { reactor }\end{array}$ \\
\hline Type A & $6.6 \%$ & $93.4 \%$ \\
Type B & $3.4 \%$ & $96.6 \%$
\end{tabular}

Table 4.4. 1: Proportion of high and low reactors within type A and type B subjects given an odds ratio of two.

basis of the meta-analysis and that calculated on the basis of the size of the odds ratio (which is roughly 2 for type A compared to type B) do agree rather well, at least for systolic blood pressure and heart rate (HRATE .23 vs .32; SYS: .36 vs .32). If one makes a less stringent assumption in defining high and low reactors, for instance by using the median split, the expected difference in the proportion of type A and B subjects in the high and low reactor groups changes from $6.6 \%$ vs $3.3 \%$ to $59 \%$ vs $41 \%$. The difference between the corresponding $\mathrm{Z}$-scores then be comes .42 .

In table 4.4.2 the expected difference between the mean of the high and the low risk subjects for a number of different odds ratios is given assuming a one-to-one relationship between risk of MI and belonging to the group of high-reactors, resulting in $5 \%$ high reactors and $95 \%$ low reactors. In the second column the expected difdference is listed when to overall proportion high and low reactors is assummed 50\% each.

High ws Low: $5 \%-95 \%$

High ws Low: $50 \%-50 \%$

$\begin{array}{lll}O R=2 & 32 \mathrm{sd} & 42 \mathrm{sd} \\ O R=4 & .64 \mathrm{sd} & .84 \mathrm{sd} \\ O R=8 & 91 \mathrm{sd} & 126 \mathrm{sd}\end{array}$

Table 4.4.2: Expected differences in degree of reactivity, expressed in standard deviation, between high and low risk groups as a function of the odd's ratio (OR) and the proportion of high vs low reactors.

Thus, a substantial increase in the expected size of the difference in reactivity may be obtained by selecting high and low risk subjects in such a way that a larger odds ratio results. For instance, when the odds ratio can be increased from 2 to 4 (assumming 20 subjects in each group, a correlation between baseline and stress of .70 and a proportion of high vs low reactors from 5-95\%) the expected difference in stress scores will increase from 32 to .64 and thus, according to equation 4.4 , the power will increase from $30 \%$ to $70 \%$. In Falger's case-control study (1989) it was shown that comparing exhausted type A subjects to vital type B subjects results in an odds ratio of 5.2 instead of 2.2 which was 
found when all type $A$ subjects were compared to all type $B$ subjects. Restricting the comparison between vital and exhausted subjects to those scoring in the lower and the upper quartile instead of using a median split, also increased the odds ratio for the exhausted subjects from 2.1 to 5.3 .

A second way to obtain larger differences in reactivity is by improving the construct validity of the classification instruments. The exclusion of subjects likely to score high for reasons unrelated to unresolved chronic conflicts, that supposedly lead to feeling of exhaustion, clarifies the theoretical meaning of the construct of vital exhaustion. Selection of subjects in this way makes the group of subjects more homogeneous. Whether it will also lead to larger differences in reactivity remains to be tested.

\subsubsection{Increase in the sensitivity of the experimental design}

The most straightforward way of increasing experimental sensitivity is by increasing the number of subjects. On the basis of equation 4.1 the number of subjects in the type $A$ and type B group needed to detect the expected difference in heart rate reactivity is 296 each, assuming a Hedges"s $g$ of .23 and critical levels of $\alpha=.05$ and of $B=.20$. There are, however, practical limits in terms of time and money to the number of subject that can be used in an experiment.

Increasing the sensitivity of the design also leads to an increase in power comparable to that of increasing the number of subjects without needing to spend additional time and money. Such an increase the sensitivity can be obtained by taking measures that will increase the correlation between values obtained during baseline and those obtained during the exposure to a stressor. The strength of the correlation between baseline values and stress values depends on the stability of the rank order of the individual scores, (i.e. the score of an individual relative to that of the other individuals). This stability, in turn, depends on whether individuals within each group respond to the tasks in the same way, whether the sequence of events between baseline and the tasks is the same for all subjects, and on the accuracy with which the dependent variables were measured. A different sequence of tasks for each subject or a high measurement error, will leadl to a lower stability and, thus, to a decrease in power without a compensatory higher difference in reactivity.

Particularly when using the interaction between group and condition as a test of the difference in reactivity between two groups, a sizeable correlation between baseline and stress values becomes important. One may remember that the reduction in variance, compared to the variance of the stress level values is $2\left(1-r_{b s}\right)$. When the correlation between baseline and stress values is smaller than .5 , the response variance is no longer smaller than the stress level variance but instead it get larger! In this case a straightforward comparison of the two groups during stress will be a more powerful test than that of the interaction between group and baseline. When using an analysis of covariance this problem does not occur since the residual variance is always smaller than the stress level variance, the reduction factor being $\left(1-r_{b s}{ }^{2}\right)$. Still, also in this type of analysis, the higher the correlation the fewer subjects will be needed to detect a given difference in reactivity. For instance, by using equation 4.4 , it can be shown that, with 20 subjects in each group and an expected difference in stress level of $32 \mathrm{sd}$, when the correlation between baseline 
and stress can be increased from .70 to 85 , the power will increase from $30 \%$ to $50 \%$. Therefore, measurement errors should be minimized, or at least assessed, in order to be able to interpret differences in reactivity between variables or conditions in a better way. Furthermore, a fixed order of experimental events is preferable even though the general task effects might be confounded with order as a result of this choice.

\subsection{2}

\section{Reducing the variability in power}

Following the above mentioned suggestions will not only increase the power in general but will also lead to less variability in power within the experiments.

The variability in power of the tests on the different dependent variables is mainly due to the variability among these variables in the correlation between the baseline and stress scores. The same is true with regard to the variability in power between the various experimental conditions. Beside the fact that some conditions may be more relevant to high risk subjects and, thus, will lead to a clearer difference in reactivity, the correlation between the baseline value and that of a particular experimental task is influenced by factors independent of the type of stressor used. For instance, the time span between baseline and the various stressors may vary. When multiple tasks and baselines are used, carry-over effects of the previous stressor may influence a baseline value in a different way, depending on the task that preceded the baseline.

Reducing the differences in correlation by increasing the measurement precision of highly instable dependent variables, and using an identical test protocol for all subjects, will lead to less variability in the power of the various F-tests that are performed to determine which variables are most sensitive to differences in reactivity or which stressors result in the largest difference in reactivity between high and low risk subjects.

\subsubsection{Reducing the number of statistical tests}

When testing the hypothesis of a larger reactivity in high risk subjects, the clearest evidence is gained when both a main task effect, indicating that exposure to the stressor led to a higher level of activation than during baseline in all subjects; and an interaction between task and group, indicating a larger difference in activation between task and baseline in the high risk group, are found. This means that for the conclusion of a higher reactivity in the high risk group, significant results on two tests, i.e. on the test for the main effect of condition and on that for the group by condition interaction, are nescessary and sufficient. When an interaction between group and condition is found without a main condition effect, it becomes important to inspect some additional F-test to understand the reason for the interaction. These include separate comparisons of the two groups during baseline and stress. Usually differences between the two groups during baseline, or changes in opposite directions will contribute to the interaction. It then becomes doubtful whether the interaction should be interpreted as evidence for higher activation during the exposure to a stressor.

Other significant tests, like an overall group difference in level of activation or a group difference in level of activation during exposure to a stressor, may be present. However, when an interaction is present, one should be very careful in using the absence of a main 
groupeffect or of a difference between groups during stress as an argument to reject the reactivity hypothesis. Due to the fact that between-subject tests are nsed when testing differences in level, while the more sensitive within-subjects tests are used when testing differences in reactivity, differences in level of activation are less easily demonstrated than differences in reactivity.

By restricting the number of F-test to those testing the main condition effect and the group by condition interaction, and only using follow-up analyses in order to understand the meaning of an group by condition interaction in case there is no main effect of condition, one reduces the risk of type I errors.

\section{5}

\section{CONCLUDING REMARKS}

Will these solutions be sufficient to minimize the risk of uninterpretable results in future experiments? I think it is wise to play it safe and combine as many of these suggestions as possible before new scientific "bets" are placed. This means that the number of questions in each experiment should be reduced while the number of subjects should be increased. One should realize that, in spite of more than a decade of research on differences in reactivity as a possible explanation for increased risk of myocardial infarction, in fact we are only just at the beginning of knowing how to pose the question in a fruitful way.

A last point to be made is whether it is still worthwhile to pursue an answer to this question. This not only depends on the feasibility of the above mentioned solutions to the methodological problems, but also to the state of the art with regard to the type $A$ behavior pattern and vital exhaustion. In other words are both psychological factors still to be regarded as risk factors of myocardial infarction?

With regard to the type A behavior pattern, a meta-analysis by Booth-Kewley and Friedman (1987) regarding the association between type $A$ behavior and heart disease showed that the association between the two was modest $(r=14)$ but, since it was based on a large number of studies $(\mathrm{N}=51)$, highly significant. Some moderators of the size of the association were suggested. A larger association between the studies that used the SI as a classification was reported and lower association was found in the studies published after 1977. Furthermore, effects based on prospective studies are smaller than those based on case-control studies. Proper tests of these differences are, however, lacking. This makes it difficult to assess the importance of the proposed moderators. Still, if not because of the suggested larger effect size, there are other reasons that make it preferable to use the SI for the selection of subjects. These are the same as those listed in the suggestions to increase the expected difference between vital and exhausted subjects, i.e. using an interview to check the reason for answering the questions may clarify the theoretical construct and lead to elimination of subjects that scored high for reasons unrelated to the presence of type $A$ behavior.

An interesting finding from the same meta-analysis is that depression, a measure closely related to vital exhaustion, was also found to be associated with heart disease. The association was at least as strong as that of the type $A$ behavior pattern $(r=.21)$ but based 
on fewer studies $(\mathrm{N}=13)$. Additional data, testing the hypothesis that vital exhaustion is a risk factor, have been gathered since our experiments started. Both the results of a prospective study by Appels (Appels et al, 1987; Appels and Mulder, 1988) and those of a case-control study by Falger (1989) indicate that this construct merits further research. From the prospective study vital exhaustion emerges as an independent risk factor similar in magnitude to blood pressure and cholesterol. The case-control study showed that vital exhaustion in the month preceding a hospital admission was reported more than twice as often by those patient who suffered from myocardial infaretion then those admitted for teasons unrelated to MI. In this study, also type A behavior was twice as often found in the cases compared with the controls. Although in this study, like in our experiments and in those of van Doornen (1988), the presence of exhaustion and type $A$ behavior were positively associated, it could be shown that both factors acted independently from each other (Falger, 1989).

Thus, at this moment, both vital exhaustion and the type $A$ behavior pattern may be regarded as independent risk factors of a similar magnitude to that of other, more somatic, risk factors like cholesterol and blood pressure. Therefore, the search for mediating mechanisms should continue. In planning such research, most of the points brought to our attention by the reviews on differences in reactivity should, however, be regarded as interesting hypotheses and not as proved facts. 


\section{REFERENCES}

Alonzo A, Simon A Feinleib M (1975). Prodromata of myocardial infarction and sudden death. Cinculation. 52: 1056-1062.

Appels A (1980). Psychological prodromata of myocardial infarction and sudden death, Psyehother Psychosom. 43: 187-195.

Appels A (1983). The year before myocardial infarction, in: Dembroski TM, Schmidt TH \& Blumchen G (Eds), Biobehavioral basis of coronary hear disease. Karger Basel.

Appels A, Hoppener P \& Mulder P (1987). A questionnaire to assess premonitory symptoms of myocardial infarction, Int J Cardiol. 17; 15-24.

Appels A, Jenkins CD \& Rosenman RH (1982). Coronary-Prone behavior in the Netherlands: a cross-cultural validation study. J Behov Med. 1: 83-90.

Appels A \& Mulder $\mathbf{P}(1988)$. Excess fatigue as a precursor of myocardial infaretion. Eur Heant $J_{\text {. }}$ 758-764.

Baker LJ, Hastings JE \& Hart JD (1984). Enhanced psychophysiological responses of Type A coronary patients during Type A-relevant imagery. $J$ Behav Med. 7: 287-305.

Barrett P (1988). Paul's Page, J Psychophysiol. 2; 301-305.

Blumentbal JA, Lane JD, Willams RB, McKee DC, Haney $\mathbb{T} \&$ White A (1983). Effects of task incentive on cardiovascular response in Type A and Type B individuals. Psychophysiology. 20: 63-70.

Bohlin G, Eliasson $K$, Hjemdahl $P$, Kleinn K \& Frankenhaeuser M (1986). Pace variation and control of work pace as related to cardiowascular, metroendocrine, and subjective responses. Blof Psychol. 23 : $247-263$.

Booth-Kewley S, \& Friedman HS (1987). Psychological predictors of heart disease: A quantitative roview. Psychol Bull, 101: 343.362.

Born GVR (1962). Aggregation of blood platelets by adenosine diphosphate and its reversal. Nature. 194: 927-929.

Brozek J, Keys A, Blackburn H (1966). Personality differences between potential coronary and non-coronary subjects. Ann NY.Acad Sci. 134: 1057-1054.

Brunson BI, \& Matthews KA (1981). Type A coronary-prone behavior pattern and reactions to uncontrallable stress: an analysis of performance, strategies, affects and attributions during failure. $J$ Pers Social Psychol. 40: $906-918$.

Buell JC \& Eliot RS (1980). Psychosocial and behavioral influences in the pathogenesis of acquired cardiowascular disease. Am Heant $J$. 100: $723-740$. 
Contrada RU, Glass DC, Krakoff LR, Krantz DS, Kehoe K, lsecke W, Collins C \& Elting E (1982). Effect of comtrol over avensive stimulation and Type $A$ behawor on cardiovascular and plasma catecholamine responses. Psychophysiology. 19: 408-419.

Contrada RI Krantz DS (1988). Stress, reactivity, and type A behavior current status and future direetions. Ann Behav Mad 10: 6470.

Contrada RJ, Wright RA \& Glass DC (1984). Task difficulty. Type A behavior pattern, and cardiovascular response. Pyehoplysiology. 21 . 638-646.

Cook WW, \& Medley DM (1954). Froposed hostility and Pharisaic virtue scales from the MMPI. $J$ Appl Psychol 38: 414-418.

Cooper T (Ed) (1981), Coronary-prone behavior and coronary heart disease: a critical review. Circulation. 63: 1199-1215.

Corse CD, Manuck SB, Cantwell JD, Giordani B \& Matthews KA (1982). Coronary-prone behawior pattern and cardiovascular response in persons with and without coronary heart disease. Psychosom Med 44:49-459.

Dembroski TM, MacDougall JM, Herd JA \& Shields JL (1979). Effect of level of challenge on pressor and heart rate responses in Type A and B subjects, J Appl Soc Psychol, 9: 209-228.

Dembrosk TM, MacDougall JM, Herd JA \& Shields JL (1983) Perspectives on coronary-prone behavior, in $\mathrm{Krantz} \mathrm{DS}$, Baum $\mathrm{A}$, Singer $\mathrm{JE}$ (Eds.) Handbook of psychology and medicine. Volume 1: Cardiovascular disease. Hillsdale, NJ: Erlbaum.

Dembrosk TM, MacDougall JM \& Shields JL (197T). Physiological reactions to social challenge in persons evidencing the Type A coronary-prone behavior pattern. J Human Stress. 3: 2-9.

Dembroski TM, MacDougall JM, Shields JL, Petitto I \& Lushene R (1978). Components of Type A coronary-prone behavior and cardiovascular responses to psychomotor performance challenge. $I$ Behav Med. 1: 159-176.

Deshmukh SV. Meyers IS \& Mouche RJ (1976). Platelet function in migraine: effect of self-medication with aspirin, Thromb Hoemost. 36: 319-325.

Diamond EL \& Carver CS (1980). Sensory processing, cardiovaseullar reactivity, and the Type A corronary-prone bebavior pattern. Biol Pschychol 10: 265-275.

Diamond EL Schneiderman N, Schwartz D, Smith JC, Vorp R \& DeCarlo Pasin R (1984). Harassment, hostility and type A as determinants of cardiovascular reactivity during competition. $J$ Behav Med. 7. 171-189.

Van Doornen LJP (1988). Physiological stress reactivity it relation to behavioral style, mood, sex, and aerobic fitness. Dissertation. Free University Amsterdam.

van Egeren LF (1979), Social interactions, communications, and the coronary prone behavior pattern: A psychophysiological study. Psychosom Med. 41: 2-18.

van Egeren LF, Abelson $\Omega$ \& Sniderman LD (1983). Interpersonal and electrocardiographic responses of Type A's and Type B's in competitive socioeconomic games. I Pychosom Res. 27: 53-59. 
Engel GL (1968). A life setting conductive to illness. The Giving-up-Given-up complex. Ann Intem Med. 89: $403-412$.

Falger PRJ (1989). Life span development and myocardial infarction: an epidemiolagical stroly, Dissertation. University of Limburg.

Fleiss JM (1986). The design and analysis of clinicol experiments. Wiley and sons.

Folkow B \& Rubinstein EH (1966). Cardiovascular effects of acute and chronic stimulation of the hypothalamic defence area in the rat. Acto Psychol Scand. 68: 48-57.

Fontana AF, Rosenberg RL, Kerns RD \& Marcus JL (1986). Social insecurity. the Type A behavior pattern, and sympathetic arousal. J Behav Med. 9: 79-88.

Forsman L \& Lindblad LE (1983). Effect of mental stress on baroreceptor-mediated changes in blood pressure and heart rate and on plasma catechollamines and subjective responses in healthy men and women. Psychosom Med. 45: 435-445.

Frankenhaeuser $\mathrm{M}_{3}$ Lundberg U \& Forsman L (1980a). Note on arousing type A persons by depriving them of work. I Psychosom Res. 24: 45.47 .

Frankenhaeuser M, Lundberg U \& Forsman L (1980b). Dissociation between sympathetic-adrenal and pituary-adrenal responses to an achievement situation characterized by high controllability: comparison between Type A and Type B males and females. Biol Psychol. 10: 79-91.

Friedman M, Byers SO, Diamant J \& Rosenman RH (1975). Plasma catecholamine response of coronary-prone subjects (Type A) to a specific challenge. Mefabolism. 24: 205-210.

Friedman M, Byers SO, Rosenman RH \& Elevitch FR (1970). Coronary-prone Individuals (Type A behavior pattern), some biochemical characteristics. JAMA. 212: 1030-1037.

Friedman M \& Rosenman R (1977). The key cause - type A behavior pattern in: Monat $J$ \& Lazarus $R$ (Eds), Stress and Coping. Columbia University Press; New York.

Friedman M, St.George $S$, Beyers SO \& Rosenman RH (1960). Excretion of catecholaminies, 17-ketosteroids, 17-hydroxycorticolds and 5-hydroxyindole in men oxhibiting a particular bethavior pattern (A) associated with high incidence of clinical coronary artery disease. $J$ Clin finwst. 39. $785-764$.

Gallacher JEJ, Beswick AD, Jones DM \& Turkington EE (1988). Type A behavior and pressor response in a representative sample of middle-aged men. I Psychosom Res. 32: 51-61.

Glass DC (1977). Behawior pattems, stress, and cononary disease. Hillsdale NJ: Erlbaum.

Glass DC, Krakoff LR, Contrada $\mathbf{R}_{\text {t }}$ Hiltoo WF, Kehoe $\mathbf{K}$, Mannucei EG, Collins $\mathrm{C}$, Snow B, Elting $\mathbf{E}$ (1980a). Effect of harassment and competition upon cardiovascular and plasma catecholamine responses in Type A and Type B individuals. Psychophysiology. 17: 453-463.

Glass DC, Krakoff LR, Finkelman J, Snow B, Contrada R, Kehoe K, Mannucci EG, Isecke W, Collins C, Hilton WF Elting E (19806). Effect of task overload upon cardiowascular and plasma catecholamine responses in Type A and B individualls. Basic Appl Soe Psychol. 1*199-218. [1,2] 
Gaks DC, Lake CR, Contrada RJ. Kehoe E Erlanger LR (1983), Stability of indiwidual differences in physiological responses to stress. Health Psychol. 2: 317-341.

Goldband S (1980). Stimulus specificily of physiological response to stress and the type A coronary prone behavior pattern. I Pers Soc Psychol: 39:670-679.

Goldstein HS, Edelberg R, Meier CF, Orzano JA \& Blaufuss L (1985). The paradoxical relation between diastolic blood pressure change under stress and the $\mathrm{H}$ factor of the Jenkins Activity Survey. $I$ Paychosom Res. 29: 419-425.

Harbin TJ (1989). The relationship between the Type A behavior pattern and physiological responsivity: a quantitative rewiew. Psychophysiology. 26: 110-119.

Hart KE \& Jamieson JL (1983). Type A behavior and cardiovascular recovery from a psychosocial stressor. J Fuman Stress. 18-24.

Heary JP \& Stephens PM (1977). Stress, Health and the Social Environment. A Sociobiologic Approach to Medicine. New York: Springer Verlag.

Holmes DS (1983). An alternative perspective concerning the differential psychophysiological responsivity of persons with Type A and Type B behavior patterns. $J$ Res Pers. $17,40-47$.

Holmes DS, McGilley BM \& Houston BK (1984). Task-related arousal of type A and Type B persons: Level of challenge and response specificity. I Pers Soc Psychol. 46: 1322-1327.

Holmes DS, Solomon S \& Rump BS (1982). Cardiac and subjective response to cognitive challenge and to controlled physical exercise by male and female coronary prone (Type A) and non-coronary prone persons. I Psychosom Res. 26, 309-316.

Houston BK (1983). Psychophysiological responsivity and the type A behavior pattern. I Res Pers. 17: 22-39.

Jenkins CD (1978). A comparative review of the interview and the questionnaire methods in the

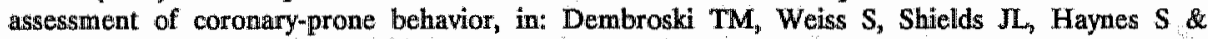
Feinleib M (Eds), Coronan-prone behavior. New York, NY: Springer Verlag, 71-88.

Jenking CD, Zyzanski. SI Resenman RH (1979), Jerkins Actiwity Survey. Manual. New York: The Psychiological Corporation.

Jennings JR \& Choi $\mathrm{S}$ (1981). Type A components and psychophysiological responses to an attention-demanding performance task Psychosom Med 43:

$475-487$.

Katz RC \& Toben T (1986). The Novacco Anger Scale and the Jenkins Activity Survey as predictors of cardiovascular reactivity $I$ Psychopath Behav Ass:8: 149-155.

Krantz DS \& Manuck SB (1984), Acute psychophysiologic reactivity and risk of cardiovascular disease: a review and methodological critique. Psychol Bull $96: 435-464$.

Krantz DS Schaeffer MA, Davia JE, Dembroski TM, Macdougall JM \& Shaffer RT (1981). Extent of coronary atherosclerosis, Type A behavior, and cardiovascular response to social interaction. Psychophysiology: 18: 654-664. 
Kuller L, Cooper M \& Perper J (1972). Epidemology of sudden death, Aneh Intem Med. 129: 714-719.

Light RJ \& Pillemer DB (1984). Summing up: the science of reviewing resturch. Harvard University Press.

Lovallo WR, Pincomb GA, Edwards GL, Brackett DJ \& Wilson MF (1986a). Work pressure and the Type A bebavior pattern exam stress in male medical student. Psychosom Med. 48: 125-133.

Lovallo WR, Pincomb GA \&. Wilson MF (1986b). Predicting response to a reaction time task heart rate reactivity compared with Type A behavior. Psychophysiology. 23: 648-656.

Lovallo WR \& Pishkin V (1980). A Psychophysiological comparison of Type A and B men exposed to failure and uncontrollable noise. Psychophysiology. 17: 29-36.

Lulofs $\mathbf{R}_{\text {y }}$ van Diest $\mathbf{R}$ \& van der Molen GM (1986). Differential reactions of type A and type B males to negative feedback about performance. I Psychosom Res. 30: 35 ino.

Lundberg U \& Forsman L (1979). Adrenal-Medullary and Adrenal-Cortical responses to understimulation and overstimulation; comparison between Type $A$ and Type $B$ persons. Biol Psychol. 9: 79-89.

Lundberg $\mathrm{U}$ \& Frankenhaeuser $\mathrm{M}$ (1980). Pituary-adrenal and sympathetic-adrenal correlates of distress and effort. I Psychosom Res. 24: 125-130.

Manuck SB, Corse CD \& Winkelman PA (1979a), Behavioral correlates of individual differences in blood pressure reactivity. I Psjchosom Res. 23 : 281-288.

Manuck SB, Craft S \& Gold KJ (1978). Coronary-prone behavior pattern and cardiovascular response. Psychophysiology. 15: 403-411.

Manuck SB \& Garland FN (1979b), Coronary-prone behavior pattern, task incentive, and cardiovascular response, Psychophysiology. 16: 136-142.

Mason JW (1971). A re-evaluation of the concept of "non-specificity" in stress theory. I Psychiatr Res. 8: 323-333.

Mathews KA (1982). Psychological perspectives on the Type A behavor pattern. Psychol Bull. 91: $293-323$

Mulder $\mathrm{G}(1980)$. The hean of mental effort. Dissertation. Groningen: University of Groningen.

Mulder G \& Mulder LJM (1981). Information processing and cardiowascular control. Psychophysialogy. 14: 392-402.

Mulder LJM (1979). INSPAN. Internal report 79-HB-439 RP. University of Groningen.

Mulder LM (1988). Assessment of cardiovascular reactivity by means of spectral analysis Dissertation. University of Groningen.

Myrtek M (1983). Typ A verialten: Untersuchungen und Literaturanalysen unter besonderer Bericksichtigung der psychophysiologischen Grunglugen. Munchen: Minerva-Publication. 
Newhin DB \& Levenson $\mathbb{R W}$ (1982). Cardiovascular responses of individuals with Type A behavior pattern and parental coronary heart disease. I Psychosom Res. 26: 393-402.

Ohman A, Nordby H \& Swebak S (1989). Components of Type A behavior and task-indured cardiovascular activation. Psychophysiology $26.81-88$.

Perkins R (1984). Heart rate change in Type A and type B males as a function of response cost and task difficulty. Psychophysiology, $21 \% 21$.

Pittner MS \& Houston BK (1980). Response to stress, cognitive coping strategies, and the type A behavior pattern, $J$ Pers Soc Fyychol $39,147157$.

Portegies M (1983) Vitale uitputting en reactiviteit. Inter Rapport. Rijksuniversiteilt Limburg:

Price KH \& Clarke LK (1978), Behavioral and Psychophysiological correlates of the coronary-prone personality. mew data and unanswered questions. I Psychosom Res. 22: 409-417.

$[1,2]$

Riedel W (1984) Confirmerende factor analyse voor effort en distress. Bijwak werkstuk Rijks Universiteit Groningen.

Rissanen V, Romo M \& Sillanen P (1978). Premonitory symptoms and stress factors preceding sudden death from ischaemic heart disease. Acta Med Scand 204: 389-396.

Rosenman RH (1978). The interview method of assessment of the coronary-prone behavior pattern, in: Dembroski TM* Weiss SM, Shields $J \mathbf{~ L}$, Haynes S \& Feinleib M (Eds), Coronary-prone behavior. New York; NY: Springer Verlag. 55-69.

Rosenthal R (1984). Meta-analytic procedures for social research. Applied social research methods series Vol.6 Beverly Hills: Sage publications.

Scher H, Hartman LM, Furedy JJ \& Heslegrave RJ (1986), Electrocardiographic T-wave changes are more pronounced in Type A than in Type B men during mental work. Psychosom Med. 48: 159-166.

Scherwitz $\mathrm{L}$, Berton $\mathrm{K}$ \& Leventhal $\mathrm{H}$ (1978). Type A behavior, self-involvement, and cardiovascular response. Pyichosom Med. 40: 593-609.

van Schijndel M, de Mey H \& Naring G (1984). Effects of behavioral control and type A behavior on cardiovascular responses. Psychophysiology. 21: 501-509.

Sthmieder $R$, Friedrich $G_{*}$ Neus $H$; Ruddel $H$ \& von Eiff AW (1983). The influence of beta blockers on cardiovaseular reactivity and type A behavior pattern in hypertensives. Psychosom Med.

45: $417-423$

Seley H (1971). The evolution of the stress concept, stress and cardiovascular disease. inx Levi $L$ (edi), Society, Stresi and Disease. The Social Environment and Psychosomatic Disease. Oxford University Press, London. 299-311.

Seley 1 (1976). The stmess of life (revised edition). MeGraw Hill New York.

Shekelle RB \& Ostfeld AM (1965). Psychometric evaluations in cardiovascular epidemiology. Ann $N Y$ Acad Sct. 126: 696-705. 
Steptoe A, Melville D \& Ross A (1984). Behavioral response demands, cardiovascular reactivity, and essential hypertension. Psychosam Med. 46(1): 33-48.

Steptoe A \& Ross A (1981). Psychophysiological reactivity and the prediction of cardiovascular disorders. I Psychosom Res. 25: 23-31.

Ursin H \& Knardahi S (1985). Personality factors, neuroendoerine response patterns, and curdiovascialar pathology, in: Orlebeke JF, Mulder G, van Doornen LJP (Eds), Psychophysiology of cardiowascular control, models, methods, and datio.

Plenum Press, New York and London. 715-732.

Verhagen F, Nass C, Appels A, van Bastelaer A \& Winnubst I/ (1980). Cross-validation of the A/B typology in the Netherlands. Psychother Psychosom. 34: 178-186.

Vingerhoets AJJM (1985). Psychosocial stress: an experintental approach Life event, coping and psychobiological functioning. Swets and Zeitlinger, Lisse.

Ward MM, Mefford IN, Parker SD, Chesney MA, Barr Taylor C, Keegan DL \& Barchas JD (1983). Epinephrine and norepinephrine responses in continuously collected human plasma to a series of stressors. Psychosam Med. 46: 471-486,

Warner ED, Hoak JC, \& Connor WE (1967). In: Platelets, their nole in hemostasis and thrombosis. 249-259.

Weidner $G$, Friend $\mathbb{R}$, Figarrotto TJ \& Mendell NR (1989). Hostility and cardiovascullar reactivity to stress in women and men. Psychosom Med. 51:

36-45.

Weiss T, Del Bo A, Reichek N \& Engelman K (1980). Pulse transit time in the analysis of autonomic nervous system effects on the cardiovascular system. Psychophysialogy. 17, 202-207.

Williams RB, Lane ID, Kuhn CM, Melosh W, White AD \& Schanberg SM (1982). Type A behavior and elevated physiological and neuroendocrine response to cognitive tasks. Science. 218: 483-485.

Wright RA, Contrada RJ \& Glass DC (1985). Psychophysiological correlates of Type A behavior. In: Katkin ES, \& Manuck SB (Eds.), Advarices in Behavioral Medicine. Volume 1. Greenwich, CT: JAI Press. 39-88.

Wu K \& Hoak JC (1974). A new method for the quantitative detection of platelet aggregates in patients with arterial insufficiency. Lancet $2: 924$. 


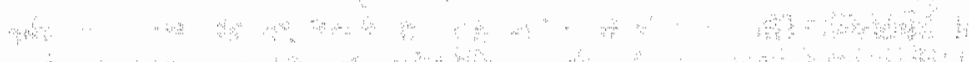

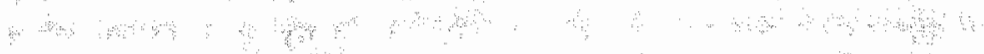

a

क से क्ष

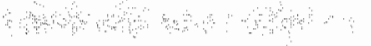

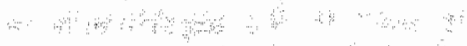

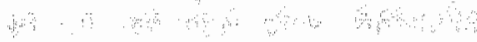

$\begin{array}{llllll} & \end{array}$

मे का क्ष

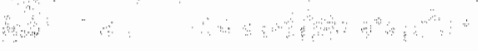

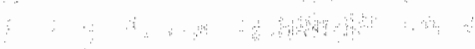

का काष

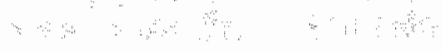

औम:

$\because$ : : : : $:$ :

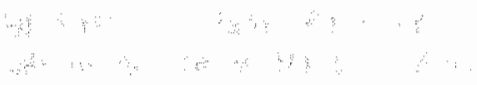

तs:

अ

$\therefore$

$\because \cdots \quad 3$

औ बह : :

तथ

$\because \quad ; \quad \forall-$

4.

औ

$\therefore \therefore$

.

$\because$ $\therefore$ 


\section{SUMMARY}

This thesis deals with the problem of how to explain, in biological terms, the association between cardiovascular disorders and two psychological constructs: the type A coronary prone behavior pattern and the syndrome of vital exhaustion.

In the first chapter a description of the two constructs is given, and results from epidemiological studies are presented. These results indicate that both type A behavior and decreased vitality should be regarded as risk factors for the development of cardiovascular disorders.

This is followed by a review of the experiments that tried to show that hyperreactivity, defined as an exaggerated response to a stressor, forms the link between type $A$ behavior and cardiovascular disorders.

Then, the main research topics of this thesis are listed. The first topic regards the biological response pattern that might be involved in the larger reactivity in type $A$ individuals. We expect that type A subjects generally show a larger increase in sympathetic adrenal medullary (SAM) activity than type B subjects when exposed to a stressor. However, we also want to see whether there are conditions in which type A subjects show a larger increase in pituitary adrenal cortical (PAC) activity than type B subjects. The second topic concerns the possible influence on hyperreactivity in type $A$ subjects of the saliency of a task, i.e. the degree to which a task has certain features that trigger the type $A$ behavior pattern. Two questions are of interest. The first question is whether the degree of saliency affects hyperreactivity in type A subjects. The second question is which experimental situations are most salient: The third topic deals with stress reactions in vital and exhausted persons. We expect exhausted subjects to show a larger increase in activation of the PAC system than vital subjects when exposed to a stressor. Furthermore, we want to see whether vital and exhausted subjects react differently in SAM activation to the various experimental conditions.

Finally the experimental approach is described in general terms.

In the second chapter an experiment is described in which the subjects are confronted with various stress-inducing laboratory tasks. Five hypotheses, derived from the main research topics are tested. The first is that type $A$ subjects generally show a larger increase than type B subjects in subjective and in the physiological activation scores that reflected SAM activity when exposed to a stressor. The second hypothesis concerns the influence of time pressure on the degree to which larger reactivity can be seen in type $A$ subjects. The third is that reactivity differences between type $A$ and $B$ subjects can be seen more clearly after criticizing the task performance of the subjects. The fourth hypothesis is that type $\mathrm{A}$ subjects also show higher levels of subjective and physiological activation than type B subjects during situations in which active coping is 
not useful or impossible. The fifth is that, when indrviduals are exposed to a stressor, variables that reflect PAC activity will show a larger change in the exhausted than in vital subjects.

The findings of this experiment may be summarized as follows: 1) The tasks did induce a general increase in subjective distress and in the amount of effort the subjects did spent. Furthermore, in all subjects the physiological activation was higher during the tasks than during the film session. However, increased platelet sensitivity or hormonal excretion could not be found during the task session. 2) No support for the hypothesis of general greater reactivity in type A subjects was found when the mean scores of the task and the film session were compared. 3) Manipulation of time pressure did not affect the type A subjects in a different way than type B subjects. 4) When criticized, type A subject showed a larger increase in subjective arousal and physiological activation than type B subjects, both when working at the task and when waiting between task periods. 5) The other conditions in which active coping was not appropriate did not lead to a difference in reactivity between type A and B subjects. 6) Particularly during the control session 17-OHCS excretion and platelet sensitivity was higher in the exhausted subjects than in the vital subjects. 7) The exhausted subjects had a lower heart rate after they had been praised for their performance. Furthermore, they showed a larger deterioration in performance accuracy, both when time pressure increased and when they were criticized.

The results are discussed and some improvements for the measurement of the dependent variables, particularly the hormonal and the platelet variables are suggested.

In the third chapter an experiment is described in which subjects are confronted with monotonous vigilance tasks to test possible differences in reactivity between type $A$ and type $B$ subjects during mental under-stimulation. These tasks have been chosen to further explore which experimental situations are suitable to trigger core characteristics of type A behavior. A number of changes were made in the way the biological vatiables are assessed, allowing a better interpretation of the results.

We expect that type $A$ individuals, compared to type B individuals, react more strongly at the start of the vigilance tasks, show, in spite of a smaller decline in physiological activation, a larger decline in performance, and have higher levels of hormones and more sensitive platelets at the and of the task. Furthermore, differences are expected to be more pronounced when the monotony of the task increases.

The major findings from this experiment are as follows: 1) The vigilance tasks did produce the expected changes: an initial rise in activation followed by a decline of activation and performance during the course of the task, 2) Some differences in vigilance behavior did occur between type A and B subjects: a larger decline in physiological activation and an earlier decline in performance was found in type A subjects, an unpredicted finding indicating that they were less able to maintain their vigilance than type B subjects. 3) These differences were not affected by the different task versions. However, the manipulation of the tasks characteristics did not affect the vigilance effects in general, and therefore, the lack of support of the saliency hypothesis can not be interpreted unequivocally. 4) No difference between task and film 
sessions in the biological variables was found, which may be partly due to the pronounced decline in activation during the tasks. 5) The level of exhaustion did not affect the vigilance effects. 6) Subjects classified both as exhausted and as type $A$, had a higher level of plasma noradrenaline regardless of the experimental condition. 7) The changes in HRV due to the tasks are independent of changes in IBI.

The chapter ends with a discussion of the findings.

In the last chapter the evidence found to support the tested hypotheses is summarized. We conclude that insufficient evidence for a generally higher reactivity in type A subjects has been found, that some evidence has been obtained for the saliency hypothesis, and that a number of interesting, but not easily explainable, findings involving vital exhaustion, did result from the analyses. These results generally are in agreement with the conclusions stated in the reviews of enhanced reactivity in type A subjects.

However, in the second part of the chapter a number of methodological objections to both the reviews and the experiments that are reviewed are presented. In most of the reviews no formal tests of the effect of factors that are supposed to influence the degree of difference in reactivity are provided. We conclude that the evidence for the effect of such factors like the choice of the classification instrument, the type of stressor used or the choice of the dependent variables, is mainiy based on post hoc analyses and that the consistency of findings is highly unsatisfactory.

In order to better understand the cause of the low consistency of the experimental results, the validity of the experimental results is examined in more detail. In order to quantify the risk of making a type I error (i.e. concluding that a difference exists when in fact it does not) and that of making a type II error (i.e. concluding that no difference exists while in fact it does), power calculations are made based on the number of subjects, the experimental efficiency, and the expected difference in response between type $A$ and $B$ individuals. The latter is obtained by performing a meta-analysis on a large number of studies on differences in stress reactivity between type $A$ and $B$ individuals. Furthermore, using meta-analytic procedures, the ideas that were presented by the reviewers regarding the influence of the classification instruments and the tasks used as stressors were formally tested.

From the power calculations it becomes obvious that most experiments, including our own, had a poor chance of finding clearly interpretable results. Not only is the expected size of the difference in reactivity very modest indeed, but also the ability to detect these differences varies considerably. This makes it doubtful whether the a priori chance of detecting differences in reactivity in different variables and during different tasks is the same. Also, the use of a large number of F-tests increases the number of both accidental significant results and chance-related negative results. Finally, no convincing evidence was found for the acclaimed superiority of the structured interview as a classification instrument or for the preference of psychomotor tasks to elicit differences in reactivity between type $A$ and $B$ subjects. Only, on a few occasions, the combination of the use of the structured interview and that of psychomotor tasks did lead to a more outspoken difference in reactivity.

The consequence of this methodological problem is that the evidence thus far 
accumulated is not sufficient to be able to make sound decisions regarding the appropriate choices of stimulus condittons and classification instrument. Furthermore, differences in the a priori chance of finding a significant effect make an explanation of the observed differences in activation pattern in basic physiological terms very difficult indeed. This hampers the right choice of the dependent variables.

Then, some measures for dealing with the methodological problem are discussed. These include 1) Increasing the size of the expected difference in reactivity between high and low risk groups by a more careful selection of subjects. 2) Increasing the number of subjects, 3) Using an experimental design that leads to a maximum in size and a minimum in variability of the correlation between baseline and stress values obtained. 4) Restricting the number of F-tests to those testing the main condition effect and the group by condition interaction.

The chapter ends with some recent findings regarding the status as risk factor of the type A behavior pattern and of vital exhaustion. The conclusion is that both constructs should be regarded as independent, additional risk factors of a similar magnitude to that of somatic risk factors like cholesterol and blood pressure. Therefore, the search for mediating mechanisms should continue. However, the conclusions from the reviews on differences in reactivity should be regarded as interesting hypotheses and not as proved facts. 


\section{SAMENVATTING}

In dit proefschrift wordt de vraag aan de orde gesteld hoe de relatie tussen cardiovasculaire aandoeningen enerzijds, en twee psychologische begrippen, te weten het type A gedragspatroon en vitale uitputting, anderzijds, door biologische mechanismen verklaard kan worden.

In het eerste hoofdstuk wordt allereerst een beschrijving gegeven van de twee psychologische begrippen en worden resultaten van epidemiologische studies aangereikt. Uit deze resultaten blijkt dat zowel type $A$ gedrag als vitaliteitsverlies een verhoogd risico op het ontwikkelen van hart- en vaatziekten met zich meebrengt.

Hierna volgt een overzicht van experimenten die gedaan zijn om te onderzoeken of hyperreactivity, gedefinieerd als een overmatige reactie op een stressor, de schakel vormt tussen type A gedrag en hart- en vaatziekten.

Dan volgen de hoofd-vragen van dit proefschrift. In de eerste vraagstelling gaat het er om welk biologisch reactiepatroon betrokken is bij de hyperreactiviteit vant de type A individuen. We verwachten dat type A proefpersonen over het algemeen een sterkere toename van activiteit in het sympaticus-bijniermerg systeem zullen vertonen dan type B proefpersonen, wanneer ze aan een stressor worden blootgesteld. We willen evenwel ook onderzoeken of er omstandigheden zijn waarin type A proefpersonen een sterkere toename van activiteit in het pijnappelklier-bijnierschors systeem laten zien. In de tweede vraagstelling gaat het om de mogelijke beïnvloeding van de hyperreactiviteit van type A personen door de mate waarin een taak saillant is. Met saillant bedoelen we de mate waarin de taak bepaalde kenmerken heeft die het type A gedragspatroon oproepen. Het gaat hierbij om twee vragen. De eerste is of de mate waarin een taak saillant is van invloed is op de mate van hyperreactiviteit in type A personen. De tweede gaat over de vraag welke experimentele situaties het meest saillant zijn. De derde vraagstelling betreft de stressreacties in vitale en uitgeputte individuen. We verwachten dat uitgeputte personen een sterkere toename van activiteit in het pijnappelklier-bijnierschors systeem zullen vertonen dan vitale personen wanneer ze aan een stressor worden blootgesteld. Voorts willen we bezien of vitale en uitgeputte personen verschillend reageren met het sympaticus-bijniermerg systeem op de diverse experimentele omstandigheden.

Tenslotte wordt de experimentele aanpak in het kort beschreven.

In het tweede hoofdstuk wordt een experiment beschreven waarin de proefpersonen diverse stress-inducerende laboratoriumtaken dienen uit te voeren. Een vijftal hypothesen, afgeleid uit de hoofd-vragen, worden getoetst. De eerste is dat type A proefpersonen in het algemeen een sterkere toename in subjectief ervaren activatie en in de waarde van die fysiologische parameters die de activiteit van het sympaticus- 
bijniermerg systeem weerspiegelen laten zien dan type B proefpersonen, wanneer ze aan een stressor worden blootgesteld. De tweede hypothese betreft de invloed van tijdsdruk op de mate waarin sterkere reactiviteit in type A personen kan worden waargenomen. De derde is dat reactiviteit verschillen tussen type A en B proefpersonen duidelijker kunnen worden aangetoond nadat de prestatie die proefpersonen tijdens de uitwoering van een taak leveren bekritiseerd is. In de vierde hypothese wordt gesteld dat type A proefpersonen eveneens hogere niveaus van subjectieve en fysiologische activatie vertonen dan type B proefpersonen gedurende condities waarin een actieve wijze van omgaan met de gestelde problemen niet nuttig dan wel onmogelijk is. De vijfde is dat, wanneer individuen aan een stressor worden blootgesteld, de variabelen die de activiteit van het pijnappel-bijnierschors weerspiegelen in sterkere mate zullen veranderen in uitgeputte dan in vitale proefpersonen.

De bevindingen van dit experiment kunnen als volgt worden samengevat: 1) De taken leidden in het algemeen tot een toename van de ervaren onlust en de hoeveelheid inspanning die de proefpersonen moesten leveren. Voorts was de fysiologische activatie in alle proefpersonen hoger gedurende de taken dan gedurende de film-zitting. Echter, een eenduidige toename van plaatjes activeerbaarheid of hormoon uitscheiding kon niet worden aangetoond, 2) Voor de hypothese van een in het algemeen sterkere reactiviteit in type A individuen werd geen steun gevonden wanneer de gemiddelde scores tijdens de stress-zitting worden vergeleken met die verkregen tijdens de film-zitting. 3) De manipulatie van tijdsdruk had geen duidelijk ander effect op type A dan op type B personen. 4) Wanneer ze werden bekritiseerd lieten type A proefpersonen een sterkere toename in subjectieve opwinding en fysiologische activatie zien dan type B proefpersonen, zowel tijdens de uitvoering van een taak als op momenten dat ze moesten wachten tussen de taken. 5) Tijdens andere situaties waarin een actieve opstelling niet toepasselijk was kon geen verschil in reactiviteit tussen type A en B personen worden aangetoond. 6) Met name gedurende de filmzitting was er sprake van een verhoogde gevoeligheid van bloedplaatjes en van een sterker uitscheiding van 17 -OHCS bij uitgeputte proefpersonen in vergelijking met vitale proefpersonen. 7) Nadat ze werden geprezen daalde de hartslag bij uitgeputte proefpersonen. Verder vertoonde deze groep een sterkere afname in nauwkeurigheid van reageren, zowel indien ze bekritiseerd werden als wanneer de tijdsdruk werd opgevoerd.

De resultaten worden bediscussieerd en enkele verbeteringen voor het meten van de afhankelijke variabelen, met name van bloedplaatjes activeerbaarheid en van hormonale activiteit worden voorgesteld.

In het derde hoofdstuk wordt een experiment beschreven waarin proefpersonen worden geconfronteerd met monotone vigilantie taken om mogelijke verschillen in reactiviteit tussen type $A$ en $B$ personen gedurende mentale onderstimulatie te testen. Deze taken zijn gekozen om verder te onderzoeken welke experimentele condities geschikt zijn om de karakteristieke kenmerken van het type A gedrag uit te lokken. Een aantal wijzigingen in de wijze waarop de biologische variabelen waren gemeten werd doorgevoerd teneinde een betere interpretatie van de bevindingen mogelijk te maken. 
We verwachten dat type A individuen, in vergelijking met type B individuen, sterker reageren bij het begin van de taak, dat ze ondanks een geringere afname in fysiologische activatie een sterkere afname in prestatie laten zien en hogere hormoon spiegels en dat ze gevoeliger bloedplaatjes hebben an het eind van de taak. Bovendien wordt verwacht dat de verschillen tussen beide types meer geprononceerd zullen zijn wanneer de monotonie van de taak toeneemt.

De belangrijkste bevindingen van dit experiment luiden als volgt: 1) De vigilantie taken brachten de verwachte veranderingen te weeg, te weten een aanvankelijke toename in activatie gevolgd door een afname van zowel de prestatie als de fysiologische activatie gedurende de taak. 2) Enkele verschillen in vigilantie gedrag tussen type A en B proefpersonen traden op: een sterkere afname in fysiologische en een eerdere afname in prestatie werden gevonden in type A proefpersonen, iets dat niet was voorspeld en er op wijst dat type A individuen minder goed in staat zijn waakzaam te blijven dan type B individuen, 3) Deze verschillen werden niet beïnvloed door de taak soort. Echter de manipulatie van de taakkenmerken had evenmin invloed op het vigilantie verloop in zijn algemeenheid, waardoor het ontbreken van steun voor de saillantie hypothese niet eenduidig geinterpreteerd kan worden. 4) Verschillen voor wat betreft de biologische variabelen tussen taak- en film-zitting konden niet worden aangetoond, hetgeen deels verklaard kan worden door de duidelijke daling van activatie gedurende de taken. 5) De mate van vitaliteitsverlies heeft geen invloed op de vigilantie effecten. 6) Proefpersonen die zowel als uitgeput als als type $A$ waren geclassificeerd hebben, ongeacht de experimentele conditie, een hogere noradrenaline spiegel in het bloed. 7) Veranderingen in hartslag variabiliteit tijdens de taak blijken onafhankelijk te zijn ván veranderingen in hartslag-frequentie.

Het hoofdstuk eindigt met een discussie van de bevindingen.

In het laatste hoofdstuk wordt het gevonden bewijsmateriaal dat de hypothesen ondersteund, nog eens samengevat. We komen tot de conclusie dat voor een algemeen verhoogde reactiviteit in type A individuen onvoldoende bewijs is gevonden, dat er enige aanwijzingen voor de geldigheid van de saillantie hypothese zijn gevonden en dat er een aantal interessante, maar niet eenvoudig te verklaren, bevindingen omtrent vitaliteitsverlies uit de analyses naar voren zijn gekomen. Onze resultaten stemmen in het algemeen overeen met de conclusies uit de overzichtsartikelen over verhoogde reactiviteit in type A proefpersonen.

Echter, in het tweede deel van het hoofdstuk worden een aantal methodologische bezwaren tegen zowel de overzichtsartikelen als tegen de daarin behandelde experimenten naar voren gebracht. In de meeste overzichtsartikelen worden geen formele testresultaten gegeven waaruit de effecten van die factoren welke geacht worden de mate van het verschil in reactiviteit te beïnvloeden, blijken. Geconcludeerd wordt dat het bewijs van de invloed van factoren als het gebruikte classificatie instrument, de keuzen van de stressor en die van de afhankelijke variabelen, voornamelijk op post hoc analyses zijn gebaseerd en dat de bevindingen weinig consistent zijn.

Om een beter inzicht te krijgen in de oorzaken van de geringe consistentie van de experimentele bevindingen wordt de validiteit ervan nader bekeken. Om een getalsmatige indruk te krijgen van het risico op zowel het maken van een type I fout (het 
concluderen dat er een verschil in reactiviteit bestaan terwijl dit niet zo is) als op het maken wan een type II fout (het concluderen dat er geen verschil terwijl dit wel zo is), worden power berekeningen witgevoerd, gebaseerd op het aantal proefpersonen, experimentele efficientie en de grootte van het te verwachten verschil in reactiviteit tussen type $A$ en $B$ indiwiduen. Dit te verwachten verschil is yerkregen door een metaanalyse te doen op een groot aantal studies over stress-reactiviteit verschillen tussen type A en B individuen. Met behulp van meta-analytisch procedures worden eveneens de ideeèn die de auteurs van de overzichtsartikelen naar voren brachten, zoals de invloed van het gebruikte classificatie instrument en de invloed van de taken die als stressor gebruikt zijn, op een formele wijze getoetst.

Uit de meta-analyse en de power berekeningen wordt duidelijk dat de meeste experimenten, inclusief die van ons, een geringe kans hadden op het verkrijgen van duidelijk interpreteerbare resultaten Niet alleen is het te verwachten verschil in reactiviteit erg bescheiden van grootte, ook de mogelijkheid om dit bescheiden verschil aan te tonen varieert aanzienlijk. Voorts is het aantal toevallig significante resultaten en het door toeval verkregen niet significante resultaten groot doordat een groot aantal F-toetsen is verricht. Tenslotte is, noch voor de beweerde superioriteit van het gestruetureerde interview als classificatie instrument, noch voor de voorkeur voor psychomotore taken om verschillen in reactiviteit tussen type A en B personen op te roepen, overtuigend bewijs gevonden. Wel leidde de combinatie van het gebruik van het interview en het aanbieden van psychomotore taken in een enkel geval tot een duidelijker verschil in reactiviteit.

Het gevolg van deze methodologische problemen is dat het tot dusverre verzamelde bewijs voor grotere reactiviteit het niet toelaat om goede beslissingen te nemen betreffende de keuze van de experimentele situaties en het te gebruiken classificatie instrument. Tevens wordt, door de verschillen in de a priori kans een significant resultat te vinden, de verklaring van de geobserveerde verschillen in activatie patronen met behulp van basale fysiologische regel systemen belemmerd. Dit bemoeilijkt vervolgens weer het maken van de keuze van de afhankelijke variabelen die in een experiment opgenomen dienen te worden.

Hierna worden enkele manieren besproken waarop deze methodologische problemen aangepalkt kunnen worden. Hieronder vallen, 1) Het vergroten van het verwachte verschil in reactiviteit tussen hoog en laag risico groepen door een zorgvuldiger selectie van proefpersonen. 2) Het vergroten van het aantal proefpersonen. 3) Het gebruik maken van een experimenteel design dat leidt tot een maximale grootte en een minimale variabiliteit in de correlatie tussen baseline waarden en stress waarden. 4) Een beperking van het aantal statistische toetsen tot die van het hoofd effect van conditie en het interactie effect van groep en conditie.

Het hoofdstuk wordt besloten met enkele recente bevindingen omtrent de status van het type A gedragspatroon en vitale uitputting als risico factor. De conclusie is dat beide constructen beschouwd dienen te worden als onafhankelijke, additionele risico factoren van een zelfde orde van grootte als die van somatische risico factoren zoals cholesterol en bloeddruk Daarom dient de zoektocht naar de tussenliggende mechanismen voortgezet te worden. De conclusies van de overzicht artikelen dienen daarbij echter als interessante hypothesen te worden beschouwd en niet als bewezen feiten. 


\section{DANKWOORD}

Allereerst wil ik mijn beide promotores en de voorzitter van de vakgroep bedanken.

Beste Ad, het feit dat dit proefschrift onder jouw hoede is voltooid, bewijst dat een crisis niet onvermijdelijk tot een breuk behoeft te leiden en ik ben je zeer dankbaar voor de inzet waarmee je mij over de eindstreep hebt weten te trekken.

Beste Bert, zowel je steun bij het verrichten van de hartslag-variabiliteit-analyse als je suggestie om me te verdiepen in meta-analytische methodes hebben tot een wezenlijke verdieping van dit proefschrift geleid waardoor ik nu met meer voldoening op de achter mij liggende werkzaamheden kan terug kijken.

Beste Joost, ondanks mijn soms zwalkend gedrag, heb je mij altijd gesteund en me de ruimte gelaten mijn eigen weg te zoeken waarbij je je als een echte Rogeriaan wist op te stellen. Mijn oprechte dank voor het vertrouwen dat je in me stelde.

Bij het verrichtten van psychofysiologische experimenten, zeker als daarbij ook nog biologische parameters van andere dan strikt fysiologische aard bepaald worden is een grote groep medewerkers van verschillende discipline onmisbaar. Mijn dank gaat dan ook uit naar niet alleen de collega's van mijn eigen vakgroep maar ook naar de medewerkers van de vakgroepen Biochemie, Farmacologie en Fysiologie en wel speciaal naar Edouard Bevers, die ons op zijn lab toeliet om daar met bloedplaatjes te stoeien, Geert Jan Tangelder die ons zijn Coulter Counter bereidwillig ter beschikking stelde, Professor Brombacher die de urinemonsters voor ons liet analyseren en Harry Struyker Boudier die het mogelijk maakte dat Mat Rousch ons de hormoonwaarden in het bloed bezorgde. Ook de medewerkers van de Instrumentele Dienst, met name Hans Koper, Wiel Schrijen en later Jeroen Hameleers, hebben door hun bereidheid steeds weer nieuwe apparaten voor reactietijd-meting en signaal-analyse voor ons te ontwerpen en te bouwen, een onmisbare bijdrage geleverd aan dit onderzoek. Dit gebeurde steeds in nawwe samenspraak met Henk Jas, eerst van de D.I.V. en later nog vaak door mij van zijn werkplek bij fysiologie weggelokt. Tijdens de dataverzameling werden de venapuncties snel en vakkundig uitgevoerd door een groep bevoegde "prikkers": Mieke, Truus, Karly, Harm en Coen bedankt daarvoor. In deze fase was Gerrie van Wunnik een steeds aanwezige vraagbaak en regelaar zonder wie alles vast niet zo goed, en zeker niet zo gezellig was verlopen. Ook zonder de "Maastrichtse mannen" zou het onderzoek niet hebben kunnen platsvinden. Alle proefpersonen mijn hartelijke dank.

Het echte werk is verricht door Margo van der Molen, Mïreille Portegies en Wim Riedel die in hun stageperiode als proefleider hebben opgetreden en er voor gezorgd hebben dat iedereen, van proefpersoon tot laborant, wist wat er van hem of haar verwacht werd. De eerst en laatst genoemden hebben hun steun zelfs tot en met de dag van de promotie plechtigheid willen voortzetten.

Tijdens en na het doen van het onderzoek heb ik veel steun gehad van collega"s en vrienden in het bijzonder van Ben Mulder, Ko Orlebeke, Lorenz van Doornen, 
Ad Vingerhoets, Lex van Bemmel, Erik en Hubert Schouten en nilet in de laatste plaats van Rob van Diest, die met niet aflatend enthousiasme en grondigheid voor mij een steumpilaar en raadsman was, en van al die anderen die mij voorzagen van tips en kritische opmerkingen.

In de afrondingsfase van het proefschrift heeft Pamela Falger op de van haar bekende nauwgezette wijze naar het grootse deel van de tekst gekeken en mij daarbij een opfris-cursus engels gegeven. Met Margriet wan Woerden en Ruud Leliveld hebben we in hoog tempo diverse versies van wordperfect erdoor gedraaid tot dat alle lay-out wensen ook echt mogelijkheden waren geworden. Margriet en Margo namen de taak op hun schouders om die mogelijkheden ook te verwezelijken tot dat wat $u$ heeft kunnen lezen.

Zonder de opvang en de stimulans van Ria zou dit proefschrift niet afgekomen zijn. Dank je voor de energie die je me steeds weer te geven wist doordat je er was.

Tot slot wil ik mijn ouders bedanken voor de steun die zij mij gegeven hebben en het geduld dat ze wisten op te brengen. Ik hoop dat het afronden van dit werk hen evenveel voldoening schenkt als het mij heeft gedaan. 


\section{CURRICULUM VITAE}

De auteur van dit proefschrift werd geboren op 15 september 1945 te Vorden. In 1964 behaalde hij het eindexamen H.B.S.-b aan het Stedelijk Lyceum te Zutphen. In 1967 begon hij met de studie psychologie aan de Vrije Universiteit te Amsterdam, waar hij in 1973 het doctoraal examen aflegde in de hoofdrichting Functieleer met als hoofdvak Psychofysiologie. Van september 1973 tot juli 1974 verrichtte hij onderzoek naar biofeedback behandeling bij migraine in het Warneford ziekenhuis in Oxford.

Per 1 januari 1975 volgde zijn aanstelling bij de Rijksuniversiteit Limburg. Aanvankelijk verrichtte hij, naast de opzet van het onderwijs en de betrokkenheid bij de behandeling van mensen met spanningshoofdpijn, onderzoek naar de invloed van fysieke inspanning op mentale taak-uitvoering. Het onderzoek waarop dit proefschrift is gebaseerd vond plaats tussen 1980 en 1984 binnen het deelproject "Psychosociale factoren bij hart- en vaatziekten". 